\title{
Ultrasonic duplex scanning in the evaluation of carotid artery disease
}

Citation for published version (APA):

Breslau, P. J. (1982). Ultrasonic duplex scanning in the evaluation of carotid artery disease. [Doctoral Thesis, Maastricht University]. Rijksuniversiteit Limburg. https://doi.org/10.26481/dis.19820521pb

Document status and date:

Published: 01/01/1982

DOI:

$10.26481 / \mathrm{dis} .19820521 \mathrm{pb}$

Document Version:

Publisher's PDF, also known as Version of record

\section{Please check the document version of this publication:}

- A submitted manuscript is the version of the article upon submission and before peer-review. There can be important differences between the submitted version and the official published version of record.

People interested in the research are advised to contact the author for the final version of the publication, or visit the DOI to the publisher's website.

- The final author version and the galley proof are versions of the publication after peer review.

- The final published version features the final layout of the paper including the volume, issue and page numbers.

Link to publication

\footnotetext{
General rights rights.

- You may freely distribute the URL identifying the publication in the public portal. please follow below link for the End User Agreement:

www.umlib.nl/taverne-license

Take down policy

If you believe that this document breaches copyright please contact us at:

repository@maastrichtuniversity.nl

providing details and we will investigate your claim.
}

Copyright and moral rights for the publications made accessible in the public portal are retained by the authors and/or other copyright owners and it is a condition of accessing publications that users recognise and abide by the legal requirements associated with these

- Users may download and print one copy of any publication from the public portal for the purpose of private study or research.

- You may not further distribute the material or use it for any profit-making activity or commercial gain

If the publication is distributed under the terms of Article $25 \mathrm{fa}$ of the Dutch Copyright Act, indicated by the "Taverne" license above, 


\section{Ultrasonic Duplex Scanning in the \\ Evaluation of Carotid Artery Disease}

Proefschrift

Ter verkrijging van de graad van doctor in de geneeskunde aan de Rijksuniversiteit Limburg te Maastricht, op gezag van de Rector Magnificus Prof. Dr. H.C. Hemker, volgens besluit van het College van Dekanen in het openbaar te verdedigen in de aula van de universiteit op vrijdag 21 mei 1982 des namiddags te vier uur.

door

Paul John Breslau

geboren te 's Gravenhage 
Promotores: Prof. Dr. J.M. Greep

Prof. D.E. Strandness, Jr., M.D.

Referenten: Prof. Dr. H.A.J. Lemmens

Prof. Dr. R.S. Reneman

K.W. Beach, Ph.D., M.D. 
Technology is nothing more or less than tools whose proper use allows us to measure and understand what is observed. Observations, following in the wake of thoughtful hypotheses, are the foundation of science, and science must be the foundation of surgery.

D. Eugene Strandness, Jr. 
The studies underlying this thesis were performed at the Department of Surgery of the University of Washington, Seattle, Wa., U.S.A. 


\section{Contents}

\section{Preface}

Chapter 1

The role of carotid artery disease in the pathogenesis of cerebral ischemia.

1.1. Anatomy.

1.2. Atherosclerosis of the carotid bifurcation.

1.3. Clinical considerations.

Chapter 2

Diagnostic approaches.

2.1. Arteriography.

2.2. Noninvasive methods.

2.2.1. Introduction.

2.2.2. Indirect noninvasive tests.

2.2.3. Direct noninvasive tests.

\section{Chapter 3}

Hemodynamics of arterial stenosis.

\section{Chapter 4}

Current status of ultrasonic techniques.

4.1. Basic Doppler and Echosystems. $\quad 17$

4.1.1. Introduction. 17

4.1.2. Continuous wave Doppler device. 18

4.1.3. Pulsed Doppler device. 19

4.1.4. Pulsed Echo imaging. 21

4.2. Analysis of the Doppler spectrum. 21

4.2.1. Zerocrossing frequency analysis. 21

$\begin{array}{ll}4.2 .2 \text {. Spectrall analysis. } & 22\end{array}$

4.3. The Duplex concept. 22

4.3.1. Introduction. $\quad 22$

4.3.2. Instrumentation requirements. 23

4.3.3. Output and recording systems. 25

$\begin{array}{ll}\text { 4.3.4. Patient examination method. } & 27\end{array}$ 


\section{Chapter 5}

Accuracy of Duplex scanning.

5.1. Introduction.

5.2. The role of common carotid artery velocity patterns in the evaluation of carolid disease.

5.2.1. Introduction.

5.2.2. Results.

5.2.3. Discussion.

5.3. The accuracy of ultrasonic Duplex scanning as compared with contrast arteriography.

5.3.1. Introduction.

5.3.2. Methods and materials. $\quad 40$

5.3.3. Results. 43

5.3.4. Discussion. 50

5.4. Effect of carbon dioxide on flow patterns in normal extracranial carotid arteries.

5.4.1. Introduction.

5.4.2. Results.

5.4.3. Discussion.

5.5. Algorithm for categorization of high grade stenoses . 68

$5,5,1$. Introduction.

5.5.2. Result:.

5.5.3. Discussion.

\section{Chapter 6}

The role of Duplex scanning in clinical practice.

6.1. Introduction.

6.2. The influence of Duplex scanning on the management of patients with suspected carotid artery disease.

6.2.1. Introduction.

6.2.2. Results.

6.2.3. Discussion. 
6.3. The impact of Duplex scanning on the evaluation of patients with asymptomatic bruits.

6.3.1. Introduction. $\quad 79$

6.3.2. Results. $\quad 80$

6.3.3. Discussion. 82

6.4. Carotid arterial disease in patients undergoing coronary bypass surgery. $\quad 84$

6.4.1. Introduction. 84

6.4.2. Results.

6.4.3. Discussion.

6.5. Velocity pattern changes following carotid endar terectomy. 88

6.5.1. Introduction. $\quad 88$

6.5.2. Results. $\quad 89$

6.5.3. Discussion. 93

\section{Chapter 7}

General Discussion.

Summary

Samenvatting

References.

Acknowledgements 



\section{Preface}

Since transient ischemic attacks are recognized as precursors of stroke and cerebrovascular insufficiency is in large part related to arteriosclerotic disease located at the carotid bifurcation, carotid endarterectomy has become one of the most commonly employed vascular operations today.

Contrast arteriography remains the ultimate diagnostic procedure for patients being considered for carotid surgery. However, the patient disconfort and risk associated with contrast arteriography limits its use for routine screening and follow-up studies. Ideally, only patients who are candidates for carotid surgery should undergo contrast arteriography and accurate noninvasive tests should be used to select these patients.

Duplex scanning, as developed at the University of Washington, is a method with the potential to detect extracranial carotid artery disease at all stages of development. The Duplex concept, a combination of B-mode and pulsed Doppler ultrasound, provides advantages over either technique used independently. In addition to audible interpretation real-time spectral analysis can be used to evaluate the obtained Doppler signals from the common, internal and external carotid artery.

The main goal of the present study is the evaluation of the clinical value of Duplex scanning combined with spectral analysis. In chapter one the role of carotid artery disease in the pathogenesis of cerebral ischemia is reviewed. Chapter two provides a review of both invasive and noninvasive diagnostic approaches concerning extracranial carotid artery disease. Chapter three introduces basic hemodynamic principles of arterial stenosis. In chapter four the current status of ultrasonic techniques and the Duplex system are described.

Chapter five contains four clinical studies, concerning the accuracy of Duplex scanning combined with spectral analysis. In the first study the value of the velocity patterns from the common carotid artery as related to disease at the carotid bifurcation will be evaluated. In the second study the accuracy of Duplex scanning combined with spectral analysis as compared with contrast arteriography was evaluated. The third was a pilot study, performed to explore a means to improve the differentiation between normal and minimally diseased carotid arteries. In this study the velocity patterns of normal subjects breathing carbon dioxide were evaluated. The fourth study was carried out to evaluate a new algorithm, designed to estimate the degree of involvement in patients with high grade stenoses.

Chapter six contains four studies, concerning the role of Duplex scanning combined with spectral analysis in clinical practice. The first and second study evaluated the influence of the noninvasive test on the managment of patients with suspected symptomatic and asymptomatic carotid disease. The third study evaluated the incidence of extracramial carotid artery disease in patients, who were candidates for coronary bypass surgery. The fourth study documented velocity pattern changes after carotid endarterectomy.

The studies of chapter five and six were the basis of the evaluation of the Duplex. scanning combined with spectral analysis. The results of these studies were the consequence of the work done by many experts in the field of ultrasound at the University of Washington during the last ten years. 



\section{Chapter 1}

\section{The role of carotid artery disease in the pathogenesis of cerebral ischemia.}

\subsection{Anatomy}

Familiarity with the anatomy of the intracranial and extracramial vasculature is essential to understand both hemodynamic changes and clinical neurological manifestations produced by extracranial carotid artery disease. The anatomical structures involved begin at the level of the aortic arch and extend to the terminations of the carotid and basilar arteries along with their potential collateral systems.

The intracranial circulation is supplied by four major extracranial vessels, consisting of the carotid and vertebral arteries on each side of the neck. On the right side, the common carotid and subclavian arteries originate from the innominate artery, the first branch of the aortic arch. The right vertebral artery begins a few centimeters beyond the origin of the right subclavian artery. The left common carotid artery is the second branch of the aortic arch and continues up into the neck as a separate trunk. The third and last major branch of the aortic arch is the left subclavian artery, which gives rise to the left vertebral artery. The right subclavian and left vertebral arteries occasionally arise directly from the aortic arch.

The course of the common carotid and vertebral arteries are similar bilaterally from the lower part of the neck upward to the circle of Willis. The common carotid arteries course upward in the neck to a point where they bifurcate into an internal and external carotid artery. The location of the bifurcation is not constant and may occur at any point within $5 \mathrm{~cm}$ of the angle of the mandible. Al the bifurcation is a dilated, wiclened area called the carotid bulb. The bulb occurs at the terminal portion of the common carotid artery and the origin of the internal carotid artery. The dilatation of the bulb plays an important role in documenting extracranial carotid artery disease both by contrast arteriography and by direct noninvasive methods.

The external carotid artery soon divides into its superior thyroid, lingual, occipital, maxillary and temporal branches. The intermal carotid artery pursues its upward course, through the petrous process of the temporal bone and the cavernous sinus. As it emerges upward out of the sinus, it gives off the ophthalmic artery, and soon thereafter the posterior communicating artery, which runs posteriorly to the posterior cerebral artery of the same side. Almost immediately it divides into the anterior and middle cerebral artery. The anterior cerebral arteries on the two sides are connected by the anterior communicating artery, which completes the circle of Willis anteriorly. The two posterior cerebral arteries complete the circle of Willis posteriorly. The size of each of these component arteries is highly variable and determines the adequacy of collateral circulation 
from one side to the other, as well as between the vertebral and the carotid systems

The most frequently described pathways of collateral circulation as a response to arteriosclerotic disease of the internal carotid artery are via the several external carotid lo ophthalmic artery anastomoses ${ }^{102}$. Additional collaterals may develop intracranially between the anterior, middle and posterior cerebral arteries via their superficial branches.

\subsection{Atherosclerosis of the carotid bifurcation.}

Extracuanal carotid artery disease is most frequently located at the carotid bifurcation, particularly at the origin of the internal carotid artery ${ }^{75}$. Although the pathological findings in atherosclerotic lesions and the principles of collateralization in the carotid system are identical to those in other arteries, it is useful in the contex of their clinical significance to describe them briefly.

Ulceration. The most widely accepted hypothesis for the pathogenesis of transient ischemic attacks resulting from involvement of structures in the internal carotid artery territory is the embolic theory ${ }^{6,1 \mathrm{k} h}$.

The release of cholesterol, calcium, platelet aggregates and thrombi into the bloodstream from an ulcerated area within a plaque can lead to botth transient and permanent neurological changes. Embolization is often difficult to prove, the best evidence found on occasion in patients with transient monocular blindness where the emboli may be seen in the retinal arteries ${ }^{126}$. In an arteriographic study Eisenberg et al. ${ }^{57}$ found demonstrable lesions (ulceration, stenosis, occlusion) in $89 \%$ of arteries in patients with hemispheric transient ischemic attacks. Amaurosis fugax, like the hemispheric transient ischemic attacks, is a warning sign of impending stroke or permanent retinal damage and should alert the physician to look for a treatable carotid lesion.

Stenosils. In many palients with hemispheric transient ischemic attacks contrast arteriography will demonstrate narrowing of the lumen at the origin of the internal carotid artery. This marrowing has been the basis for the hemodynamic theory of transient ischemic allacks. In general, stenoses can be divided in clinical terms into those that reduce tolat blood llow and those that do not ${ }^{\text {s5y }}$. An increasing atterial stenosis does not reduce blood flow and distal pressure until a critical degree of stenosis is reached 22,177 . In the carotid system flow reduction is not thought to occur until there is an internal diameter reduction of more than $50 \%$ of the vessel ${ }^{51}$. Whenever a flow or pressure reducing lesion is considered to be the cause of transient ischemic attacks, the stenosis should reduce the diameter by at least $50 \%$ in combination with an inadequate collateral system.

Occlusion. A total occlusion of the internal carotid artery is the most advanced lesion pathologically. A slowly developing occlusion may occur in the absence of manifest symptoms or signs of cerebral insufficiency ${ }^{53}$. The explanation for asymptomatic occlusions is the development of available collateral pathways including the extracranial carotid arteries and intracranial communicating vessels ${ }^{2 *, 102}$. Knox et al. ${ }^{112}$ found in 45 patients with an unilateral occlusion of the internal carotid artery focal neurological symptoms in 26 , the remaining 19 palients did not have any symptoms of cerebral insufficiency. 


\subsection{Clinical Considerations}

The average age of patients with manifestations of cerebral ischemia is 60 years with twice as many men are affected as women ${ }^{62}$.

Hypertension is found in about half of these patients, approximately $15 \%$ have diabetes mellitus. The clinical manifestations of an ischemic episode usually appear suddenly, and the full clinical picture develops within a matter of minutes or hours. Focal dysfunction in a territory of a cerebral artery lasting from a few minutes to 24 hours, returning to the preattack status is referred to as transient ischemic attack (TIA). Episodes of TIA are recognized as precursors of stroke since approximately $40 \%$ of affected patients are likely to have a cerebral infarct within the first five years after the initial event. This is more than 10 times the rate of expected stroke incidence for persons of the same age and sex in a general population ${ }^{172}$. Furthermore, at least $75 \%$ of patients with completed stroke will give a history of previous transient ischemic attacks ${ }^{115.5}$.

Data from the Joint Study Project ${ }^{75}$ indicated that $74 \%$ of patients with ischemic stroke syndromes have at least one significant stenotic lesion in the extracranial vasculature at a surgically accessible site. Since Thompson et al. ${ }^{16 \% .169}$ presented their long-term results, carotid endarterectomy has become accepted therapy for selected patients with cerebrovascular insufficiency. In general most authors in the recent literature will agree that carotid endarterectomy in symptomatic patients can be performed with a mortality rate of $1 \%$ to $2 \%$ 7\%.171.17\%.

Medical treatment as a means to prevent stroke in patients with amaurosis fugax or focal transient ischemic attacks began with the introduction of effective antihypertensive therapy. The coincidence of the declining incidence of stroke ${ }^{10 *}$ with the introduction of effective antihypertensive therapy suggests that this treatment may be the most important cause of the decline is. Anticoagulants were introduced in the 1950's. Despite all the clinical trials performed, Barnett ${ }^{1 *}$ stated in the Robert Wartenberg Lecture: "From all those trials it cannot be concluded unequivocally that anticoagulants are helpful. Similarly it cannot be concluded that anticoagulants have no place in TIA management." Platelet inhibiting drug therapy (aspirin) seems to have a potential place in the prevention of stroke as hats been demonstrated in controlled trials ${ }^{\mid 7,61}$.

Although there is still disagreement as to what the treatment of choice should be in the prevention of stroke, the growing interest in this areat has created a demand for both accurate invasive and noninvasive methods to detect carotid disease and assess its potential as a cause of symptoms. 


\section{Chapter 2}

\section{Diagnostic approaches.}

\subsection{Arteriography.}

In 1937 Egar Moniz ${ }^{123}$ demonstrated the value of arteriography in the diagnosis of thrombosis of the internal carotid artery. This report, although primarily concerned with the arteriography of the intracerebral lesions, called attention to the association of hemiparesis and internal carotid thrombosis. Direct needle puncture of the common carotid artery and injection of contrast material became a standard technique in the diagnosis of carotid arterial disease.

In 1953 Seldinger introduced the arterial catherization over

a wire guide ${ }^{151}$, this method permitted selective injection of all vessels of the aortic arch. Seldinger"s technique gained wide acceptance and still is the preferred technicue in most centers. It is a relatively simple method and permits visualization of all components of the intra and extra-cranial arterial circulation.

With the establishment of contrast arteriography follow-up studies by repeat contrast arteriograms were reported to assess progression of arterial occlusive disease of the carotid arteries. Javid et al. ${ }^{91}$ reported a study of 93 patients who underwent repeated contrast arteriography with a mean follow-up period of 3 years. In $38 \%$ of these patients, there was no evidence of progression of arterial disease, in $62 \%$ marked progression was noted. The rate of progression in this study appeared to be related to hypertension, cerebral symptoms, and the degree of stenosis. Because contrast arteriography, like any invasive technique, presents a small but reall risk with respect to morbidity and mortality ${ }^{80}$ the method is generally considered to be impractical to utilize on a repetitive basis in follow-up studies for quantifying progression of disease.

Complications due to cerebral contrast arteriography can be divided in major and minor complications. Hass et al. ${ }^{75}$ reported a mortality rate of $0.7 \%$ in a series of 4748 patients with cere brovascular insufficiency, in which complete four vessel studies were made. The stroke rate in this study was $0.5 \%$, minor complications occurred in $5.3 \%$. In a multicenter retrospective study Swanson el al. "164 reported $0.6 \%$ permanent neurological complications and $13 \%$ transient complications in 1328 patients undergoing cerebral arteriography. Wylie and Ehrenfeld 14. reported a mortality rate of $0.4 \%$ and a stroke rate of $0.5 \%$ in a series of 800 patients undergoing four vessel studies using the Seldinger technique.

Neurological events related to extracranial carotid artery disease occur both in the presence of high grade lesions (hemodynamic theory) and minimal lesions (embolic theory). It is therefore important to review the reliability of arteriographic studies in detecting both minimal and severe lesions. In general most surgeons have found that arteriography underestimates the amount of disease found at the time of operation. For the coronary arteries this problem was 
highlighted by Staiger et all. 154

In 203 coronary arteries in which the cross-sectional area was measured directly, post mortem anteriograms underestimated the extent of involvement in $47 \%$. In a post-mortem study of 64 carotid bifurcations Croft et al ${ }^{47}$ found that the agreement between two observers on the $x$-ray appearances was $87.5 \%$ and between the agreed $x$-ray appearences and the macroscopic pathological findings $78.6 \%$. This study shows nicely that even in post-mortem studies the disagreement about the actual diameter reduction at the level of the carotid bulb is considerable.

Hass et al. ${ }^{75}$ concluded that in reading arteriograms, estimating the degree of stenosis of the internal carotid artery was more accurate when more than $50 \%$ of the diameter was involved. Edwardset al. "4 evaluated the accuracy of angiographic diagnosis of carotid artery ulcerations in 50 carotid arteries. In $34 \%$ of the cases there was distgreement between the surgical observations and the radiological findings. In a recent study by Chikos et al. "the intraobserver and interobserver agreement reading 128 multiplanar selective arteriograms of the carotid arteries were evaluated. The intrabserver agreement for the internal carotid artery was $75 \%$ for a vessel with $0 \%$ stenosis, $90 \%$ for a vessel with a 50 $99 \%$ diameter reduction, and $97 \%$ for a total occlusion when the same reader estimated the diameter reduction wice. The interobserver agreement the probability that a second reader would agree with the first reader) for the internal carotid artery was $57 \%$ for a vessel with $0 \%$ stenosis. $85 \%$ for a vessel with a 50 99\% diameter reduction, and $97 \%$ for a total occlusion. Overall it is obvious that there is considerable variability in reporting results of reading carotid arteriograms related to the number of radiologists involved and the number of times an individual radiologist evaluates the results.

The development of noninvasive methods of diagnosing extracranial arterial disease has highlighted the problem associated with reading arteriograms. Since contrast arteriography traditionally has been the method used for the diagnosis of extracranial arterial disease, it is generally accepted as the "gold standard" in evaluating the accuracy of the noninvasive tests. The previously described variability studies with regards to arteriography however have highlighted a serious problem, especially in patients with no or minimal disease. This variability is one of the reasons that total agreement between arteriography and noninvasive tests ramains a problem to be considered.

Recent innovations in computer technology have permitted the delopment of digital subtraction arteriography as a new method in the diagnosis and management of patients with arterial disease ${ }^{175}$. Preliminary results ${ }^{162}$ for the detection of extracranial carotid artery disease are promising.

Turnipweed ef al. ${ }^{176}$ reported that they found a close relation between intravenous digital subtraction and standard arteriography images of the carotid arteries.

Intravenous digital subtraction arteriography will clearly find a role in the overall management of patients with extracranial carotid artery disease. The method does not require hospitalization, may be used for repetitive testing, and has less risk than conventional contrast arteriography.

However digital subtraction arteriography must still be viewed as a potentially simpler method of obtaining views of the carotid artery. In that respect it will not 
be a better "gold standard" than conventional studies. Furthermore, since venous arteriography is invasive, it will still pose risks. It is also unlikely that the method will be suitable for follow-up studies in order to document progression of disease. These research oriented follow-up studies should be preceded by accurate noninvasive tests.

\subsection{Noninvasive methods.}

\subsubsection{Introduction.}

Since stroke is the second most common cause of death due to vascular disease, a variety of noninvasive tests have been developed

to evaluate arterial occlusive disease of the extracranial carotid arteries.

Furthermore, the extracranial carotid system is an attractive system for

noninvasive studies for the following reasons: (1) the disease is usually localized at the carotid bulb and the first few centimeters of the internal carotid artery; (2) the relatively superficial location of the extracranial carotid system makes it accessible to direct ultrasonic methods; and (3) more advanced lesions produce changes in pressure and flow, resulting in the development of collateral pathways which may be evaluated by indirect methods. The techniques used for noninvasive diagnosis are most readily separated into those which are termed indirect and those which are termed direct.

Techniques which are designated as indirect, test for hemodynamic changes at some point removed from the site of involvement. Since the ophthalmic artery is the first branch of the intracranial carotid artery, obstructive lesions at the carotid bifurcation may produce pressure and flow changes in the orbital branches of that artery and its periorbitall ramifications. Supraorbital directional Doppler examinations ${ }^{13,38,116}$, pulse-delay oculoplethysmography ${ }^{94,95}$, and pressure oculoplethysmography ${ }^{55,68,70}$ are considered to be indirect methods. Indirect tests are the most common noninvasive techniques employed today.

Direct tests provide anatomical or hemodynamic information concerning the carotid artery itself. Phonoangiographic analysis 52.94 .314 , velocily waveform analysis $122.134,135.148$, ultrasonic Doppler imaging ${ }^{87,84,138,153}$ and Duplex scanning 11.12 are considered to be direct methods.

\subsubsection{Indirect noninvasive test.}

\section{Supraorbital Doppler Examination.}

The supraorbital directional Doppler examination, as initially des cribed by Brockenbrough ${ }^{3.8}$ and modified by Barnes et al. ${ }^{13}$ uses a direction sensing Doppler system to evaluate flow direction in the medial frontal or supraorbital arteries. Reversed flow in the frontal artery indicates extracranial collateral flow in the presence of a significant stenosis (flow reducing lesion). In the technique described by Barnes, compression of an external carotid branch may produce diminution, obliteration or reversal of flow in the medial frontal artery if this artery is the source of collateral flow to the brain. Low common carotid compression is also utilized to identify contralateral carotid or intracranial collaterall flow. Although accurate when positive, this method alone is an 
unsutable diagnostic test for the patient with focal symptoms. since these are olten due to emboli from ulcerations in plaques which are not large enough to produce a pressure gradient, and thus do not result in the development of collateral circulation $15,32,114$.

\section{Pulse-delay Oculoplethysmography.}

The method takes adwantage of the fact that the opthalmic artery is the first branch of the internal catotid artery. The system, developed

by Kartchner and McRae ${ }^{64,45}$ utilizes plastic corneal cups filled with water applied to the eyes with a negalive pressure of $40-50 \mathrm{~mm} \mathrm{Hg}$. A pulse wave representing the volume change of ocular filling is generated for each eye and recorded on light sensilive paper. An electronic comparison is performed between the two wavelorms, yielding a differential tracing which allows the examiner to compare the rate of ocular filling between the two eyes. In addition,

photoplethysmographic clips are applied to each ear lobe to record the pulse arrival time from the external carotid artery in order to detect external carotid stenosis or occlusion. Bilateral internal carotid stenoses are detected by comparing the ocular with the ear pulse waveforms.

Kartchner and McRae ${ }^{\%}$ presented data from their experience of 1287 patients evaluated by OPG combined with carotid phonoangiography (CPA), 295 of whom underwent arteriography. Of these 295 patients, 147 were noted to have arteriographic flow reducing lesions and 148 non-flow reducing lesions. Of the 147 "positive" arteriograms, 16 had a negative OPG for a false negative rate of $11 \%$. Of the 148 "negative" anteriograms, 17 had a positive OPG for a false positive rate of $11 \%$. In a clinical report, Blackshear et al. ${ }^{25}$ presented data from 191 patients evaluated by OPG-CPA at the vascular laboratory of the University of Washington. There were 106 sides available for comparison with arteriography. Results of the examination were negative in 47 of the 58 vessels that were either normal or had a stenosis of less than 50 percent diameter reduction, resulting in a lalse positive rate of $19 \%$. All eight total occlusions of the internal carotid artery had positive tests. However, only 17 of 40 vessels with high grade lesions exhibited. an ocular pulse delay, so the false negative rate was $58 \%$ for this category. These results as obtained by Blackshear indicated that the method is inadequate to screen patients suspected of having carotid artery disease. Keagy et al. ${ }^{\text {g7 }}$ reviewing 291 carotid arteries that were subjected to the pulse-delay $O P G$ concluded, like Blackshear, that this method was inadequate to accurately screen patients.

\section{Pressure Oculoplethysmography.}

The technique of pressure oculoplethysmography, as developed by Gee et al. ${ }^{68,69.70}$, utilizes scleral eye cups placed dateral to the cornea and held in place with a negative pressure of $300 \mathrm{~mm}$ of mercury which obliterates ocular pulsations. There is a direct relationship between the degree of vacuum applied and the resulting introcular pressure. If the intraocular pressure exceeds the ophthalmic artery pressure, the blood flow to the eye will stop and no pulsations will be recorded. The onset of the pulsations occurs when the systolic arterial 
pressure exceeds the intraocular pressure, allowing the determination of the systolic arterial pressure in the ophthalmic artery.

Baker et al. "reviewed their results for detecting carotid stenosis using the pressure OPG in 227 patients who underwent biplanar artenography. Their overall accuracy in the correct identification of hemodynamically signilicant stenoses (more than $60 \%$ decrease in luminal diameter) was $93 \%$. Eikelboom io presented data from the experience of 1200 OPG tests, from which 390 could be compared with contrast arteriography. In this material 106 patients had hemodynamically significant stenoses (stenosis of $65 \%$ or more). The sensitivily of the pressure OPG tests was $89 \%$ with a specificity of $84 \%$.

Pressure oculoplethysmography seems to be an accurate method in detecting high grade lesions. There are basically two serious limitations of the method: (1) the method is not able to detect non-pressure/ flow reducing lesions which can be the cause of embolic focal neurological symptoms; and (2) the method is unable to differentiate between a high grade stenosis and a total occlusion, a distinction which is of clinical importance.

\subsubsection{Direct noninvasive test.}

\section{Velocity Waveform Analysis}

Most authors have used velocity waveform analysis as an indirect method, andyzing the tracings from the common carotid artery to estimate the involvement of disease in the internal carotid artery 134.148 . More recently welocity waveform analysis is used as a direct method, analyzing the tracings from the carotid bifurcation $^{94}$.

Velocity wave form information from the extracranial carotid arteries can be obtained both by continuous wave and pulsed Doppler systems. Where continuous wave systems provide an analog output of one tracing, multigated pulsed Doppler systems have the potential to display a number of velocity tracings from several points across the artery simultaneously ${ }^{\mathrm{ss}}$.

Planiol and Pourcelot ${ }^{134}$ using a continuous wave device introduced the resistivity index (RI) of the common catrotid artery as a means to predict extracranial carotid artery disease. This index is a ratio of peak systolic velocity and diastolic velocity, the RI is normally between 0.5 and 0.75 . A RI greater than 0.75 is considered to be pathological. For total occlusions and stenoses with a diameter reduction of more than $90 \%$ the method seems to be accurate. A more complex method was introduced by Rutherford et al. " ${ }^{\text {at }}$. Using a continuous wave device, five hand measured parameters were obtained from the analog output of the velocity tracing of the common carotid artery. They found a $100 \%$ accuracy of separating normal from diseased carotid arteries, the discrimination between stenoses greater than and less than $50 \%$ diameter reduction was $82 \%$. As will be discussed, this method has great potential particularly when a computer can be utilized to carry out the complex discriminant analysis which is required ${ }^{7 / 3}$.

The use of multigated pulsed Doppler devices in obtaining simultaneous velocity tracings over the cross-section of the blood vessel have been reported by Keller et 
al. ${ }^{3}$ and more recently by Hoeks ${ }^{14}$. Although the results from clinical studies are not yet avaibable, this method will find a place in screening of patients with suspected extracranial carotid artery disease.

\section{Carotid Phonoangiography (CPA)}

This technique developed by $K$ artchner and $\mathrm{McRae}{ }^{44}$ for use in conjunction with the oculoplethysmograph utilizes a handheld microphone which is placed over the carotid artery in low, middle and high locations. A photographic display of the bruit in relation to the first and second heart sounds is produced. In addition, the bruits may be analyzed audibly by the examiner during the course of the study. In the study presented by Blackshear et al. ${ }^{25}$ carotid phonoangiography either alone or in combination with pulse-delay oculoplethysmography was not of sufficient accuracy to recommend its use as part of a noninvasive evaluation of carotidartery occlusive disease.

\section{Phonoangiographic Specural Analysis (P.A.)}

Quantification of the frequency spectrum of phonoangiographically recorded carotid bruits has been reported by Duncan et al. ${ }^{52}$ By Fast Fourier Transform (FFT) spectral analysis, they identified a single "break frequency" beyond which the intensity of all higher frequencies sharply declined. The diameter of the residual lumen (dp) within the stenotic vessel can be calculated from the equation $d p=U / f_{i j}$, where $U$ is the peak systolic velocity in the unoccluded portion of the artery and $f_{0}$ is the observed break frequency derived from the spectral analysis. The peak systolic velocity ( $U$ ) is assumed to be a constant of $500 \mathrm{~mm} / \mathrm{sec}$. The commercial edition of the phonoangiograph was recently evaluated in the vascular laboratory at the University of Washington ${ }^{104}$. One hundred and sixteen carotid bruits were analyzed, with biplanar contrast arteriography available for comparison in 43 instances. The diameter of the vessel at the site of the stenosis estimated by phonoangiography and contrast arteriography were compared and found to agree within $1 \mathrm{~mm}$ of each other in $85 \%$ of the cases. Phonoangiography provides an accurate method to estimate the residual lumen of the carotid arteries whenever a bruit is present. It is important to realize however, that only $13 \%$ of the patients in the vascular laboratory at the University of Washington were referred because of an asymptomatic bruit ${ }^{60}$.

\section{Contimuous Wave Uhrasonic Arteriography}

The combination of a position sensing arm and a continuous wave Doppler device with a storage oscilloscope permits visualization and identification of the major arteries in the neck and allows the examiner to interrogate the characteristics of flow from precise points along the carotid arteries ${ }^{109,138,153}$. Clinical results using this method are reported by Weaver ef al. ${ }^{177}$. These authors noted that the method had no predictive value in detecting stenoses that fell in the $0-50 \%$ range. With $50-75 \%$ stenoses, the Doppler accuracy was $52 \%$, increasing to $71 \%$ when 76-99\% diameter reducing stenoses were present. The fact that the correlation between ateriography and ultrasonic imaging was poor in the group of patients with stenoses less than $50 \%$, is a serious disadvantage of the method. Curry and 
White ${ }^{48}$ introduced the color coded ultrasonic arterial scanner (Echoflow), using a continuous wave Doppler device. The Doppler shifted signals scattered by the blood at peak velocity are detected by filters with varying frequency ranges. signals from each filter activated are color-coded. The color selected at any point, corresponds to the highest frequency filter passing a Doppler signal. Although the display differs from the continuous wave ultrasonic arteriograph, the principles of the method are basically the same. White and Curry ${ }^{17 \%}$ presented their results with the Echoflow in 424 carotid bifurcations. There was no differentation between normal and minimally diseased internal carotid arteries. In the $75-99 \%$ diameter reduction range the Doppler accuracy was $80 \%$.

\section{Pulsed Doppler Ultrasonic Arteriograph}

The pulsed Doppler ultrasonic arteriograph as initially developed by Hokanson et al. ${ }^{87.88,124,125.157}$, consists of a transducer containing a $4 \mathrm{~mm}$ piezoelectric cystral mounted on a position sensing arm, a pulsed Doppler ultrasonic flow detector, and a storage oscilloscope. Whereas the continuous-wave Doppler velocity detector senses blood flow anywhere in the path of the ultrasound beam, the pulsed Doppler is able to sample from a small region in space at any desired distance from its transducer (see 4.1.3.).

Sumner et al. 163 reported the agreement between contrast arteriography and Hokanson's ultrasonic arteriography in 209 carotid arteries. Using a diameter reduction greater than $20 \%$ as criterion for a positive test, the sensitivity of the method was $81 \%$ and the specificity was $88 \%$. Blackshear et al. ${ }^{24}$ evaluated the results with this method in 66 carotid sides also studied by contrast arteriography. There were 8 arteriographic normal arteries, 9 with a diameter reduction less than $10 \%, 23$ with a $10-49 \%$ diameter reduction, 22 with $50-99 \%$ diameter reduction, and 4 with total occlusions. With the pulsed system there was one false positive $(12 \%)$. Audible changes in the Doppler signal compatible with turbulence were detected in 13 of the 22 vessels $(59 \%)$ with a $10-49 \%$ diameter reduction, and in 20 of the 22 vessels with a $50-99 \%$ dianeter reduction $(91 \%)$. Three out of the four total occlusions were diagnosed correctly. Pulsed Doppler imaging, although far from perfect, represents a promising development in the field of noninvasive technology. It has the ability to detect not only total occlusions and high gracle lesions but also, particularly in combination with spectrum analysis, lesser degrees of stenoses.

\section{B-mode linaging}

Olinger ${ }^{130}$ has made the first two-dimensional images of peripheral blood vessels using a pulsed echo system. Bernstein et al. ${ }^{123}$ used ultrasonic imaging for detecting and following abdominal aortic aneurysms. Cooperberg et al 4 reported the use of a $B$-mode scanner in detecting extracranial carotid arlery disease. Out of 52 carotid bifurcations evaluated, they confirmed the arteriographic diagnosis in $44(85 \%)$. They found false-negative results in the cases where the plaques were not calcified. Recently Hobson et al. ${ }^{83}$ reported the accuracy of a B-mode imaging system with and without the additional use of a Hokanson ultrasonic arteriograph. The most dramatic difference between the B- 
mode imaging system and the ultasonic arteriograph was the poor accuracy of the $\mathrm{B}$-mode echosystem to predict total occlusions. Out of 17 arteriographic proven occlusions only 3 were identified correctly by the B-mode imaging system.

An important limitation of pulsed-echo systems as has been shown in the results presented by Hobson, is that a totally occluded internal carotid artery can appear lo be patent from the B-mode ultrasound examination. This limitation was carly appreciated by Hartley et al. They showed a wide variation in the attenuation characteristics of plaques dependent on the presence or absence of calcium. In the case of soft, fatty plaques reflection is similar to that of blood, while calcificd plaques showed an increase in intensity by a factor of 1000 over that of a normal vessel wall. Whenever an internal carotid artery is occluded without the presence of calcium, the occluded artery will not differ from a patent vessel on the B-mode image, hence the occlusion will not be identified. These findings motivated the development of the Duplex scanner which combines real-time Bmode imaging with pulsed Doppler ultrasound.

\section{Ulranonic Duplex Scanning.}

The Duplex concept is based upon the ability to define the walls of the vessel within a two-dimensional image and uses the pulsed Doppler to evaluate the blood velocities with in the artery visualized. "This concept was integrated into the system described by Barber et al. 11,12. The first prototype available for clinical studies $1,32,137$ was used in the noninvasive laboratory at the University of Washington until Janury 1980 . The accuracy of this method combined with spectral analysis in detecting extracranial carotid artery disease as compared with contrast arteriography was reported by Fell et al. ${ }^{54}$. In this study 135 patients underwent contrast arteriography giving 270 carotid bifurcations available for comparison. The agreement between contrast arteriography and ultrasonic Duplex scanning combined with spectral analysis was: $35.5 \%$ (4/11) for a normal bifurcation; $45 \%$ $(9 / 20)$ for a diameter reduction less than $10 \% ; 58 \%(52 / 90)$ for a $10-49 \%$ diameter reduction; $81.5 \%(84 / 103)$ for a $50.99 \%$ diameter reduction; and $70 \%$ (32/46) for a total occhision. In January 1980 the prototype scanner was replaced by a commercially avalable Duplex scanner*. The improvements made were in large part the results of previous experience and projected needs (see 4.3.).

\section{: ATL Mark V Duplex Scanner.}




\section{Chapter 3}

\section{Hemodynamics of arterial stenosis.}

Utrasonic Doppler techniques wilize the Doppler effect to make an estimate of flow velocities ${ }^{149}$. The velocity profiles are reflections of hemodynamic characteristics of the ardiovascular system. Changes in the velocity profles will be more meaningtul with the knowledge of basic principles of fluid dynamics. Fon that reason some of these hemodynamic principles will be introduced in this chapter.

Fluid energy. The driving force for flow in the circulation is not the clifference in pressure but the difference in total fluid energy . The total fluid energy (E) is expressed as the sum of the pressure energy $(\mathbb{P})$, gravitational potential energy (pgh), and kinetic energy $\left(1 / 2 \mathrm{pV}^{2}\right)$.

$$
\mathrm{E}=\mathrm{P}+\mathrm{pgh}+\mathrm{t} / 2 \mathrm{p} \mathrm{V}^{2}
$$

where $p=$ the density of the fluid

$\mathrm{g}=$ the acceleration due 10 gravity

$\mathrm{h}=$ the depth

$V=$ the velocity of the fluid.

The difference in totalluid energy between two points in the same streamline are expressed in the Bernouilli equation:

$$
\mathrm{P}_{1}+\mathrm{pgh}+1 / 2 \mathrm{pV}_{1}^{2}=\mathrm{P}_{2}+\mathrm{pgh}_{2}+1 / 2 \mathrm{pV}_{2}^{2}
$$

providing that the flow is steady and the system frictionless.

Energy losses. Because of friction in the human circulation, with resultant energy dissipation as heat, the Berr ouilli equation requires modification with the addition to heat. There are two forms of energy losses due fo blood flow: (1) inertial losses, due to changes in blood flow velocities or direction, and (2) viscous losses, as a resull of blood viscosily.

Viscous energy losses are expressed by Poisetille's law, which states that the pressure gradient along a tube $(\triangle P)$ is proportional to the mean velocity $(V)$, the tube length (L) and the fluid viscosity ( $\pi$ ) and inversely proportional to the second power of the tube radius ( $R$ ):

$$
\Delta P=V\left(8 L \pi / R^{2}\right)
$$

From this equation has come the familiar relationship: pressure = flow $\mathrm{x}$ resistance, which is analogue $100 \mathrm{hm} \mathrm{s}^{\mathrm{s}} \mathrm{aw} \mathrm{w}^{15 \mathrm{j}}$. It is important to reallize that 
Pousouilles's law applies only to the idealized conditions of steady (non pulsatile). laminar (non turbulent) flow of a Newtonian fluid (viscosity unaffected by velocity).

Laminar and turbulent fow. Reynolds ${ }^{142}$ was the first to precisely describe the conditions under which the transition from laminar to turbulent flow occurred. $\mathrm{He}$ demonstrated that at higher flow rates the fluid became more sensitive to disturbances, until a critical point was reached where laminar flow could not be maintained. It was observed that the point at which this transformation occurred was dependent upon the vessel diameter, the mean velocity, the density and viscosity of the fluid. These factors can be expressed as the dimensionless quantity called the Reynolds number:

$$
\operatorname{Re}=\frac{\bar{V} \operatorname{di} \rho}{\eta}
$$

where $\mathrm{Re}=$ Reynolds number

$\mathrm{V}=$ the mean velocity of the flow

$\mathrm{di}=$ the internal diameter of the artery

$\rho=$ the density of blood

$\eta=$ the viscosity of blood

AI Reynolds numbers approaching 2000 , a disturbance is necessary to institute turbulence in a smooth pipe. The larger the Reynolds number, the less disturbance is necessary to cause this turbulence ${ }^{180}$. Although the use of Reynolds number helps to understand the circumstances in which turbulence can occur, it must be emphasized that the calculation of Reynolds number should be strictly applied only to conditions of steady llow.

Velocity profiles. Newtonian fluicls under conditions of steady laminar flow develop parabolic velocity profilles. In the mammalian arteries however the profiles are seldom true parabolic. The fact that blood flow is pulsatile prevents the formation of at truly parabolic profile. The explanation for this is provided by McDonald ${ }^{i x}$. Near the wall, blood moves more slowly than that of the center stream. Because of this the direction of flow near the wall is more sensitive to variations in the pressure gradient than the flow in the more central laminae. Consequently when flow reversal begins, it starts at the wall. During periods of the pulse cycle blood can reverse close to the wall, whilst it continues to flow in a forward direction at the center of the stream. As the pulse rate increases, there is no time for the development of a parabolic profile and the profile becomes flattened (plug llow).

Pulsatile flow. In spite of the fact that pulsatile flow seems less efficient than steady flow, the matmmalian organism seems to function best under these circumstances ${ }^{1019}$. Although phenomena like energy losses and turbulence are much more complex in pulsatile flow than in the situation of steady flow, the basic principles stay the same. 
Energy losses due to pulsatile flow has been estimated $10 \%$ in the descending aorta, $16 \%$ in the abdominal aorta, and $24 \%$ in the carotid artery as compared with steady flow". These losses are largely a result of the acceleration of blood at certain points, the occurrence of disturbed flow patterns and the non-Newtonian character of blood ${ }^{112}$.

Does turbulence occur in the normal pulsatile arterial system? The pulsatile nature of flow should encourage flow disturbances since at peak flow rates the critical Reynolds number $(2000)$ is often exceeded. Attinger at al. "were able to demonstrate that eddies, vortices, and turbulence may occur in the cardiovascular system, particularly during flow deceleration and at branch points. The energy losses associated with these flow instabilities appear to be insignificant with respect to average fllow rates. For that reason Altinger preferred to refer to the appearance of eddies and vortices as "disturbed llow". "The term disturbed flow. describes the intermediate state between laminar flow and fully developed turbulence.

In normal pulsatile flow the deceleration phase after peak systole is the most unstable phase of the pulse cycle. Whenever flow disturbances develop, they are first observed during the deceleration phase of systole ${ }^{\prime \prime !}$.

Stenosis. As fluid passes from a tube of larger diameter through one of smatler diameter, it must accelerate to a higher velocity in the narrow segment to maintain the same total flow. With this acceleration offluid, potential energy (pgh) is converted into kinetic energy $\left(1 / 2 \mathrm{pV}^{2}\right)$ and a pressure drop occurs.

$$
\Delta \mathrm{P}=1 / 2 \mathrm{p}\left(\mathrm{V}_{1}^{2}-\mathrm{V}_{2}^{2}\right)
$$

At the distal end of the narrow segment there is a deceleration of velocities, where kinetic energy is converted into potential energy. This last conversion is not complete however, with a total pressure drop over the marrow segment as the result. The situation described above can only be calculated accurately for steady non-pulsatile flow, although the same principle applies to pulsatile llow.

Both experimental and clincal investigations indicated that it is necessary to bave a marked stenosis of an artery before the blood llow is reduced. May et al. ${ }^{17}$ investigated the effect of short graded stenoses on the flow and pressure through ilac arteries of anaesthetized dogs. In their study il was necessary to constrict the luminal area by $80 \%$ before a significant decrease in arterial flow and pressure occurred. May pointed out that each organ system has its own characteristic critical stenosis.

Deweese el al. measured pressures proximal and distall to anteriosclerolic plaques in the internal carotid artery. They found that in the carotid system a stenosis of greater than $63 \%$ minimal lumen diameter, leaving a channel less than $1.0 \mathrm{~mm}$ in diameter, always produced a drop in pressure and flow. As a general rule a critical or "hemodynamically significant" stenosis of the internall carotid artery is considered to have a diameter reduction greater than $50 \%$.

When blood flows through a stenosis the velocities of the bloodcells will increase in the stenosis. In the post-stenotic segment the movement of the red cells 
adjacent to the wall are retarded as compared with those in centerstream and vortices will be present. With the increase of midstream velocities due to an increase of the stenosis, the movement of red cells adjacent to the wall are more retarded as compared with those in the centerstream and flow disturbance will be present.

Both changes in peak systolic velocities and eddies caused by flow disturbance, which occur with increasing stenoses, can be identified by pulsed Doppler techniques in combination with spectral analysis ${ }^{20165}$.

Bruits. It is presently considered that bruits can be created by periodic and unfformly oriented vortical eddies produced when a rapidly moving fluid stream encounters an obstacle. Given a source of vortices, a rise in velocity of blood stream will increase the loudness of the murmur and raise its pitch. The spectra of the turbulent pressure fluctuations have been shown to be similar to those in vitro pipe turbulence ${ }^{117}$. A method of arterial bruit analysis based on the analysis of turbulent flow allows estimation of the extent of arterial stenosis at the site of origin of the bruit ${ }^{52}$.

The characteristics of turbulent flow within a vessel are such, that the intensity of the sound produced declines as frequency increases beyond a single identifiable frequency (the break frequency).

This break lrequency allows the evaluation of the residual lumen diameter from the equation:

$$
\begin{aligned}
\mathrm{dp} & =\mathrm{U} / \mathrm{to} \\
\text { where } \mathrm{U} & =\text { peak systolic velocity } \\
\mathrm{fo} & =\text { break frequency } \\
\mathrm{d} p & =\text { residual artery lumen }
\end{aligned}
$$

Kistler et al. "101 found in 31 out of 33 arteriographic proven stenoses a residual diameter estimated by phomoangiography agreement within $0.5 \mathrm{~mm}$. A study pertormed in the vasculat laboratory of the University of Washington ${ }^{104}$ has shown, that this form of bruit analysis first reported by Duncan el al. ${ }^{52}$, provides an aceurate method to estimate the residual lumen of carotid arteries. It is important to recognize that $10 \%$ of the bruits heard with a stethoscope in the study by Knox et al. "1hit could not be analyzed using this device. 


\section{Chapter 4}

\section{Current status of ultrasonic techniques}

\subsection{Basic Doppler and Echo systems}

\subsubsection{Introduction}

The first clinically orientated application of Doppler ultrasound was by Satomura ${ }^{149}$. Although red blood cells are weak reflectors (only $0.02 \%$ of the transmitted ultrasonic energy is reflected), they can be recognized by their motion. Many subsequent investigations have confirmed the utility of this method in providing useful data concerning flow patterns in the arterial and venous system.

The mechanism is based on the principle of the Doppler effect, which is a change in frequency of an acoustic or ultrasonic wave that results when the total path length between the transmitting and receiving transducer is changing. For convenience, the change in ultrasound frequency is referred to as "Doppter frequency", which can be expressed as in the following equation:

$$
\Delta f=\frac{2 f o v \cos \varnothing}{c}
$$

where:

$$
\begin{aligned}
& \Delta f=\text { Doppler frequency }(\mathrm{KHz}) \\
& \mathrm{fo}=\text { transmitting frequency }(\mathrm{MH} / \mathrm{z}) \\
& \mathrm{v}=\text { velocity of red blood cells }(\mathrm{cm} / \mathrm{sec}) \\
& \varnothing=\text { angle between the Doppler beam and the axis of the vessel. } \\
& c=\text { velocity of sound in soft tissue }(\mathrm{cm} / \mathrm{sec})
\end{aligned}
$$

The equation shows that the angle between the Doppler beam and the axis of the vessel must be known to calculate the velocity of moving red blood cells. (see figure 1).

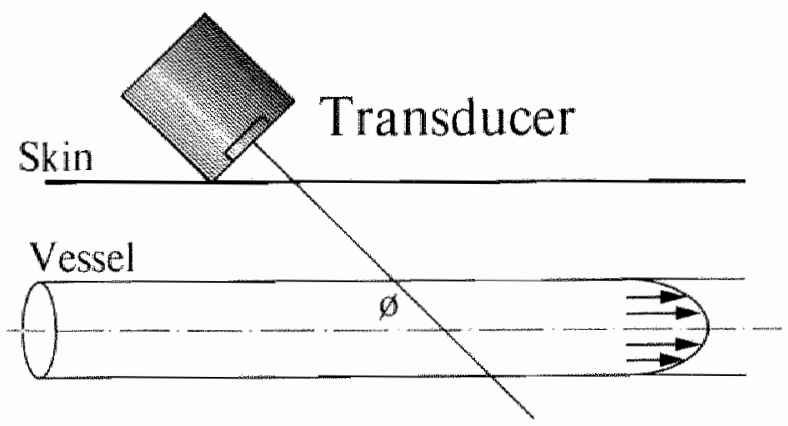

Figure 1.

Schematic cross section of ult rasound iransduce whose sound beam intercepts a blood vessel at the angle $\emptyset$. The different values of velocity across the vessel are shown by the length of the velocily vectors. 
When an ultrasonic Doppler instrument is used to detect the Doppler shift due to moving red blood cells, not just one frequency but many different frequencies are produced. The Doppler frequencies are spread in a "Doppler spectrum". The most important cause of different frequencies in the Doppler spectrum is the variation in velocities of red blood cells within the vessel ${ }^{137}$. For example red blood cells close to the wall move, due to friction, more slowly than those centerstream. This is shown in figure 1 , where each arrow represents a velocity vector whose length is proportional to the velocity.

Changes in the vessel wall will produce changes in the Doppler spectrum, which can be used to detect arteriosclerotic lesions ${ }^{2 t, 27}$. This will be discussed in detail later (see 4.1.3.).

The equipment used for the detection of velocity operates with either a continuous wave or pulsed Doppler system. Both are described in the following paragraphs.

\subsubsection{Continuous wave Doppler device.}

A continuous wave instrument usually has two piezoelectric crystals, one for transmission of ultrasound and one for reception of the backscaitered signals. The velocities of all red blood cells within the ultrasonic range of the instrument will be defected (figure 2). The Doppler spectrum of the continuous wave instrument will for that reason be relatively broad.

Figure 2.

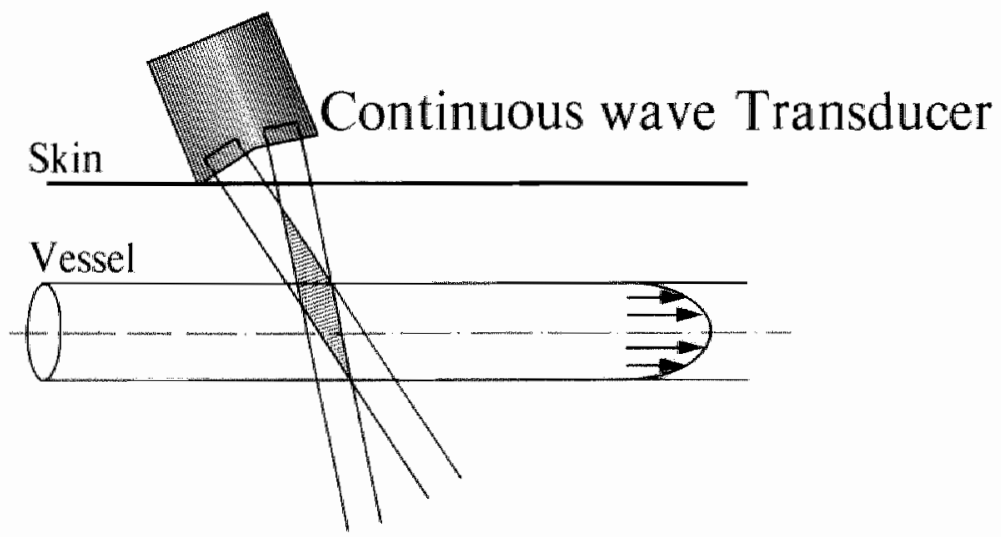

Schematic cross scetion of transtucer and sound beany shape for a continuous wave Doppler system. All velocities across the vessel are deteeted.

Early Doppler systems and many in use today are "non-directional", in other words there is no distinction made between targets moving away from the probe and those moving towards the probe. These systems can only be used to detect the presence or absence of flow and are frequently used in pressure measurements.

The ability to detect the direction of flow was a marked improvement in the. Doppler instruments. At the University of Washington Nippa et al. ${ }^{127}$ developed a phase rotated quadrature mode for bidirectional information. This system is based upon the introduction of a $90^{\circ}$ phase shift in the detection process of the Doppler signals. Two channels are generated each having forward and reverse flow information. The phase relation between the two channels contains the directional 
information. The quadrature method is applicable both for continuous wave and pulsed Doppler systems.

\section{1.3. Pulsed Doppler device.}

A pulsed Doppler instrument contains one piezoelectric crystal used both for transmission and reception of ultrasound ${ }^{20}$. Short pulses of ultrasound are repeatedly transmitted into the tissue by the piezoelectric crystal. The pulses are spaced in order to permit one pulse to retturn before the next transmission.

At a selected time following transmission the signal from the piezoelectric crystal is gated for further processing into the Doppler signal ${ }^{7.8 .9}$. By selecting the time between transmission and gating one can choose the range or depth at which velocities are detected. The velocities detected by the device are an average of all velocities within a small region called "sample volume" ${ }^{0,3}$. The size and shape of the sample volume depends on the duration of the transmitted burst, transducer bandwidth and receiver bandwidth ${ }^{84}$. The depth at which the velocity is detected is controlled by the amount of delay in the nange gate.

Since the pulsed Doppler device only measures velocities within its sample volume the Doppler spectrum will contain only partial information of velocities within a vessel (figure 3 ). With this system it is possible to place the sample volume in the centerstream of the vessel where most of the red blood cells have the same velocity, resulting in a narrow band of frequencies. When turbulence occurs the spectrum of frequencies will be broad. Moving the sample volume from centerstream closer to the wall will, even in normal vessels create broadening of the Doppler spectrum due to velocity gradients which are present near the wall.

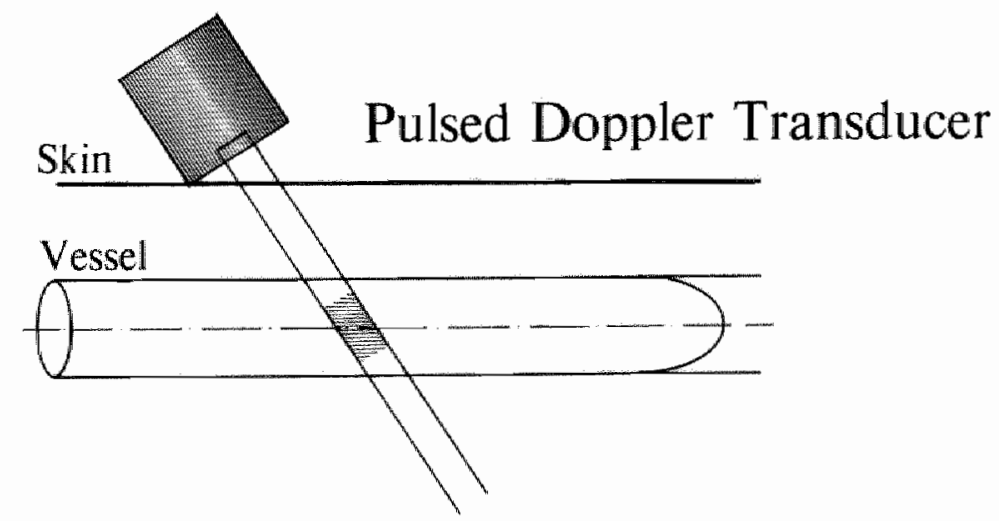

Figure 3.

Schematic cross section of transducer and sample volume for a pulsed Doppler system. Only the velocilies within the sample volume are detected.

The dual capacity (transmission and receiving) of the instrument forces a compromise in determining the best pulse repetition rate (PRF). Doppler shift frequencies above one-half of the pulse repetition rate in the forward direction appear in the reversed direction. For example with a PRF of $4 \mathrm{KHz}$, the maximum forward Doppler shift detected is $2 \mathrm{KHz}$. With Doppler shifts covering $4 \mathrm{KHz}$, tho 
first $2 \mathrm{KHz}$ will appear in forward direction, the other $2 \mathrm{KHz}$ will appear in the reverse direction. This phenomona is referred to as "aliasing" of the signal (figure 4).

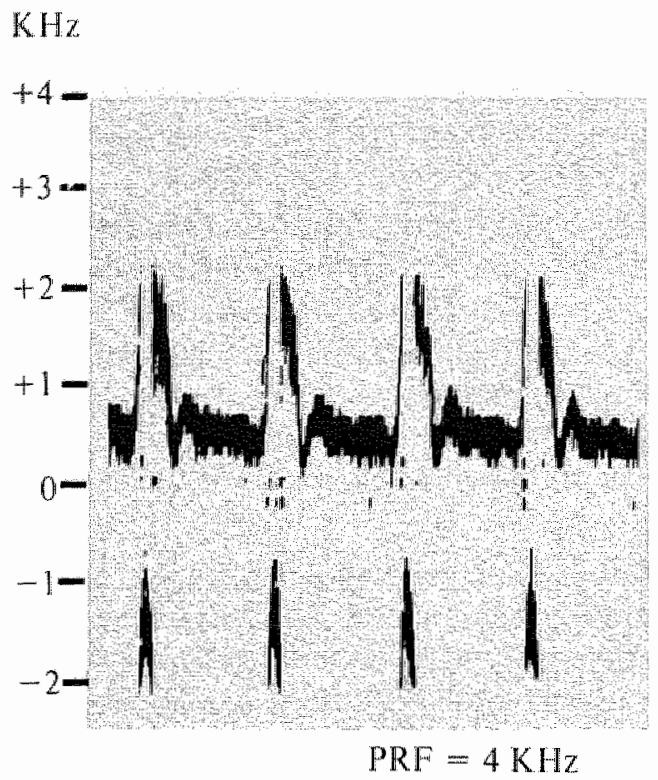

Vugure 4

Directional aliasing at a PRF of $4 \mathrm{KHz}$.

Frequencies abowe $2 \mathrm{KHz}$ appoar in the reverse diraction.

Detection of disturbed flow in the arterial system has great potential as a means of identifying changes due to early atheromatous lesions. The hot film anemometer hats proven a useful instrument in demonstrating disturbed and turbulent flow both in animals and model studies ${ }^{7 !}$. A sensitive noninvasive method which could be used to detect and possibly quantitate flow disturbances would be of great value.

The comparison of a pulsed Doppler ultrasonic velocimeter with a hot film anemometer in measuring disturbed flow in models has shown a good correlation between the two techniques ${ }^{165}$. The visuat manifestation of disturbed flow, as detected by a Doppler velocimeter, is an increase in the width of the spectrum of the back scattered signal. This is due to greater variation in velocities of blood cells within the "sample volume" of the pulsed Doppler device. Hutchinson et at. ${ }^{\text {(h) }}$ demonstrated that disturbances downstream of graded stenoses caused a pattern of increased spectral width in the heart-cycle. This was found in the phase of flow deceleration with mild stenoses but occurred earlier in the pulse cycle as the degree of narrowing was increased. Thiefe's and Hutchinson's studies have shown that pulsed Doppler instruments have the potential to detect early flow disturbances, they are the basis for estimating the degree of diameter reduction in extracranial carotid artery disease.

A major problem in pulsed Doppler operations is placing the sample volume at a desired anatomical site in a three dimensional arterial system. This problem can be solved by using multiple receiving gates set at different depths ${ }^{39.85}$. or by using an imaging system to position the sample volume within the vessel of interest ${ }^{87.138}$. 


\subsubsection{Pulse Echo Imaging.}

Before the development of the Doppler method, ultrasonic imaging systems were introduced. These systems are based, like radar, on the time of flight of sound and the strength of the reflector. In the early systems, a stationary collimating transducer was used to send an ultrasound pulse into tissue. The reflections in time, from successively deeper tissue planes, were plotted on an oscilloscope with depth plotted horizontally and amplitude of the reflected wave plotted vertically. This amplitude versus depth plot was called amplitude or "A" mode.

An A-mode display can be converted to an intensity mode display by retaining the depth coordinate as dimension atong a lime and plotting the strength of reflection as intensity. The intensity can be either two level or providing a full range of scale. When properly adjusted, the level of gray can be used to identify tissue structures.

Moving structures can be followed in real time with both A-mode and intensity mode, which motion mode is referred to as "M" mode $\mathrm{M}^{74}$. A two dimensional image can be made by a similar system. The transducer is moved over the skin while the intensity mode line is moved over a display screen, painting a 2 dimensional image. Such a display is called a "B" mode image. In early systems. the B-mode probe was moved over the skin by hand. Now the ultrasonic transducers are moved mechanically or electronically over the image field producing a full field display in at fraction of a second, thus creating "real-time" ima$\operatorname{ging}^{20}$.

\subsection{Analysis of the Doppler spectrum.}

The analysis of the audible Doppler signalls by an experienced technician hats been successful in the detection of pathology in the arterial system ${ }^{15}$. In order to document the velocity information, a hardcopy of the audible Doppler signals should be available. In principle two melhods of display are used to obtain a hardcopy of the audible signal: (1) zero-crossing frequency analysis; and (2) spectral analysis.

\subsubsection{Zero-crossing frequency analysis.}

The method is based on the electronic detection and marking of zero-crossing: events of the Doppler signal. The electronic device may only count positive, negative, or both positive and negative crossings of the Doppler signal. The zerocrossing principle is traditionally used to obtain an analogue velocity tracing. displaying only the "mean" of the whole Doppler spectrum. The analog tracing gives the "avaraged" velocity as an instantaneous function of time. The advantages of the method are its simplicity and the fact that the hardcopy can be obtained using a simple strip chart recorder. However several disadvantages should be known by the user of this method: (1) signals with a poor signal-tomoise ratio cannot be adequately recorded"13 ${ }^{1 / 2}$ since only the "mean" of the Doppler spectrum is displayed primarily artefacts maly also be recorded and interpreted as pathological finding ${ }^{22}$ and; (3) since all information of the Doppler spectrum is reduced to one frequency, a certain amount of potential important information is 
lost for further analysis ${ }^{12}$. More information of the Doppler spectrum can be oblained when spectral analysis is used ${ }^{19,72}$.

\subsubsection{Spectrall analysis.}

Spectral analysis the method of choice for processing the Doppler signals from peripheral arteries, since it retains all the Doppler information and allows the examiner to determine if potentional artefacts are present. These artefacts can be the result of: (1) background noise; (2) high amplitude, low frequency signals arising from the arterial wall or probe movement; and (3) simultaneous recordings made from arteries or veins.

Spectral andysis may be accomplished by an off-line method. The Kay sonograph was originaly such a method, it was reliable but time-consuming and directional Doppler information could not be displayed. Real-time spectral analysis is preferable and may be performed by multiple bandpass filter analysis ${ }^{16.144}$, time compression analysis $^{42}$, or fast Fourier transform analysis ${ }^{77.145}$. Real-time spectral analysis is easily avaliable and has found its place in many noninvasive laboratories $20,4,7,72,134$.

Spectral analysis of continuous-wave Doppler signals can be used to determine a maximum frequency envelope, but an assessment of disturbed flow based on "spectral broadening" is not possible because the continuous-wave signal contains velocities from the entire flow profile. The wide range of velocities sampled with the system produces a broad frequency spectrum from both normal and diseased vessels. A pulsed Doppler system, as built in the Duplex scanner, samples flow from specific siles and avoids the disadvantages of the continuous wave instruments.

\subsection{The Duplex concept.}

\subsubsection{Introduction.}

Olinger made the first two dimensional images of peripheral blood vessels, using a pulsed-echo system. An important limitation noted with such a system was, lhat a lotal occlusion of the internal carotid artery appeared to be patent from the B-node images. A tissue study of atheromatous deposits, found within and along diseased arteries, by Hartly et al. ${ }^{76}$ showed a wide variation in the absorption characteristics of plaques dependent on the absence or presence of calcification. These lindings motivated the development of the Duplex scanner which combines real-time B-mode imaging with pulsed Doppler ultrasound. The Duplex concept is based upon the ability to define the walls of a vessel with a two dimensional image and utilizing pullsed Doppler ultrasound to evaluate the velocity chatracteristics of the flow within the visualized artery. These concepts were integrated into the Duplex Scanner II system described by Barber et al. "1.12, the first prototype atvalable for clinical studies 132,133 was used until January 1980. This device amploys three $5 \mathrm{MHz}$ ultrasonic tranducers mounted on a rotating drum encased within a water-filled silastic boot used for generating a real-time Bmode image. Within the silastic boot, a single gate $5 \mathrm{MHz}$ pulsed Doppler with a 
quadrature output is mounted in the plane of the B-mode image. The beam path af the pulsed Doppler is represented on the image by an enhanced white line. while the point from which the flow is sampled is represented by a white dot. The clinical evaluation of this scanner was reported by Blackshear et al. ${ }^{24}$ and Fell ex al. ${ }^{59}$.

Since the introduction of the method, the system had undergone continuous modification to improve not only on the ease of the study but the accuracy of the technique in predicting the presence or absence of carotid artery disease. In January 1980 the prototype system was replaced by a commercially avalable device. The improvements made then were in large part the results of previous experience and projected needs.

\subsubsection{Instrumentation requirements.}

A block diagram of the Mark V Duplex scanner system is shown in figure 5 . The basic elements include: the scan head assembly, pulse echo electronics, scanhead controller, pulsed Doppler electronics, and associated display and reconding instrumentation. Three fixed focus $5 \mathrm{MHz}$ transducers imbedded in a rotating wheel generate a two dimensional image of soft tissue interfaces with 16 levels of grey. The sector angle of the B-mode image can be choosen at $45^{\circ}, 60^{\circ}$ or $90^{\circ}$. Routinely a $60^{\circ}$ sector angle is used. The image rate is 30 frames per second. Since the image information is digitized the B-mode image can be "frozen", in which mode one of the three crystals is used as a pulsed Doppler. This permits the use of a pulse repetition frequency of $₫ 9.2 \mathrm{KHz}$ which, given a certain depth, allows at Doppler frequency response up to $9.6 \mathrm{KHz}$ before aliasing occurs (see 4.1.3.).

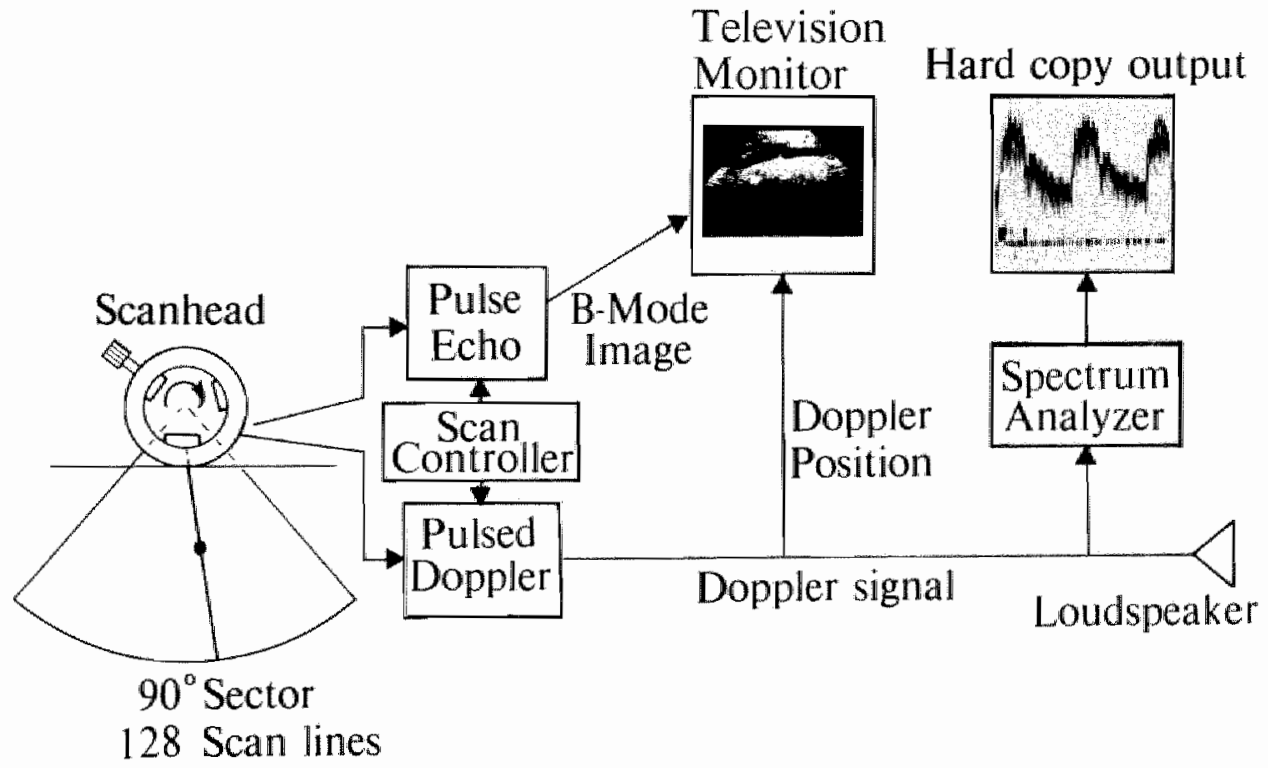

Figure 5.

Block diagram of Mark $V$ Duplex scanner. The basic elements includes: scanhoal assembly, pulse echo electronics. scanhead controller, pulsed Doppler electronics, and associated display and recording instrumentation. 
The B-mode image is displayed on an oscilloscope screen. The position of the Doppler beam is indicated by a white line superimposed on the $\mathrm{B}$-mode images in order to be able to measure the angle between the axis of the vessel of interest and the Doppler beam. A small dot represents the position of the sample volume along the Doppler beam. Both Doppler angle and sample volume depth are adjustable by controls on the scan head. Hardcopy of the Doppler signals from at normal common carotid artery from different angles demonstrates nicely that whenever comparison is made between studies, the angle between the Doppler beam and the axis of the vessel should be known (see 5.2.1.). The differences between the signals at $45^{\circ}-60^{\circ}-90^{\circ}$ can be explained by the Doppler equation, where the Doppler frequency changes with the cosine of the angle between the Doppler beam and the axis of the vessel (see 4.1.1.).

Two different scan heads are commercially available, the medium focus scan head and the short focus scan head. The medium focus scan head has a designation CCC, operating $5 \mathrm{MHz}, 9.5 \mathrm{~mm}$ crystal diameter and a $40 \mathrm{~mm}$ focal point. The short focus scan head has a designation $\mathrm{CBB}$, operating at $5 \mathrm{MHz}, 6 \mathrm{~mm}$ crystal diameter and a $20 \mathrm{~mm}$ focal point ${ }^{3}$.

The pulsed Doppler sample volume can be defined as the region in space in which the detection of the movement of the blood cells occurs ${ }^{5,93}$. The use of a pulsed system allows this sample volume to be positioned at any desired depth from the transducer.

Since the width of the ultrasound beam (W) varies as a function of range, the sample volume is likewise range dependent. The beam widths at different ranges from the two scan heads are shown in figure 6 and 7 . These plots were obtained using a hemispherical point reflector ${ }^{103}$. Results from such studies have been shown to provide beamwidths similar to those obtained with moving scatterers in water tank studies ${ }^{6,1,3}$. The pulse echo techniques were employed as they are easier to perform. The beamwidth of the medium focal scan head is narrowest from $351045 \mathrm{~mm}$ in its focal range, whereas that of the short focus scan head is narrowest from $201030 \mathrm{~mm}$. Therefore the short focus scan head is appropriate Cor studies of vessels which lie less than $30 \mathrm{~mm}$ from the transducer, and the medium focus scan head is more appropriate for vessels lying further than $30 \mathrm{~mm}$ from the scan head. The arteries in the neck lie within $30 \mathrm{~mm}$ of the skin surface in the majority of human subjects. Therefore, the short focus scan head should on theoretical basis, be used for all subjects of normal build. Since this study was performed ${ }^{4 / 3}$, the short locus scan head has been used routinely in the vascular laboratory at the University of Washington.

Additionally, the size of the sample volume, relative to the size of the vessel being studied, will have a considerable effect on the velocities which are recorded If the sample volume is large relative to the vessel diameter, than a greater distribution of velocities will be detected. This results in an increase of the spectral width. The use of pulse echo techniques have allowed the calculation of the relative sizes of the sample volumes of the 2 scan heads. The beam widths for the medium and short focus scan heads are $5.5 \mathrm{~mm}$. and $2.0 \mathrm{~mm}$ respectively at the $20 \mathrm{~dB}$ level at a range of $25 \mathrm{~mm}$ (figure 6 and 7 ). The corresponding range measurements for the five cycle gated burst is $2.3 \mathrm{~mm}$ for both scan heads. The sample volumes for the 
short and medium focus scan heads are calculated to be 3 mm and $24 \mathrm{~mm}^{3}$ respectively. This calculation is based upon the $20 \mathrm{~dB}$ beam width measurements. a sample and hold window of one micro second and an oblate spheroid used as the geometrical model. It is apparent that the medium focus scan head will sample a much greater volume and potentially a greater range of velocities than the short focus scan head. This will be demonstrated by the results of the clinical studies (chapter 5).

\section{Medium Focus}

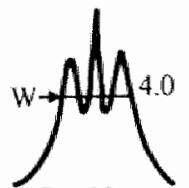

$\mathrm{R}=20 \mathrm{~mm}$

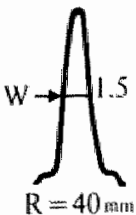

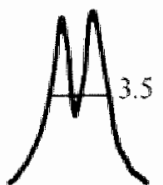

$25 \mathrm{~mm}$

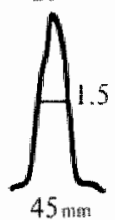

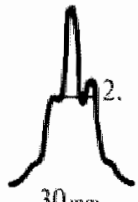

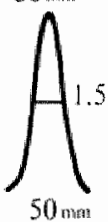

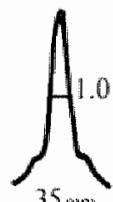

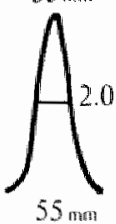

Figure 6.

The beam plots for the medium focus scanhead. These were generated from a 2 .4 dianneter stainless stecl hemisphere. The width of the the beam $W(\mathrm{~mm})$ is shown for the ranges $R(\mathrm{~cm})$.

\section{Short Focus}
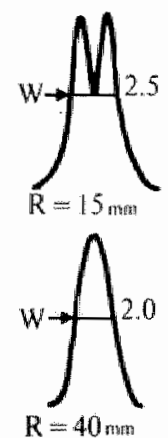
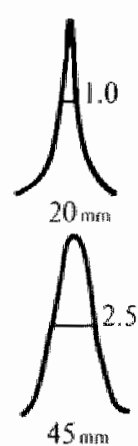
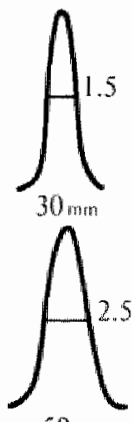

50 mon
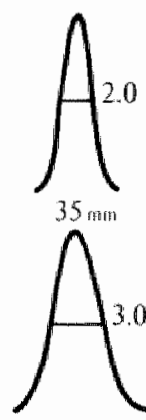

$55_{\text {, }}$ :11

Figure 7 .

The beam plots for the short focus scanhead. These we re generated from a 2.4 diameter stecl homphere. The width of the beam $W(m m)$ is shown for the ranges $R$ (cm).

\subsubsection{Output and recording systems.}

The quadrature outputs of the pulsed Dopplen signal are sent to a Fast Fourier Transform spectrum analyzer. The initial work with the Duplex scanner utilized a time compressing method (Honeyell model SAI-51C spectrum analyzer). More recently a Fast Fourier Transform (FFT) has been used (Honeywell research prototype FFT Spectral Analyzer). This digital device operates on-line and provides a full scale frequency display of $10 \mathrm{KHz}$ (amplitude versus frequency). 
The maximum frequency range of the spectrum analyzer is $12.8 \mathrm{KHz}$, constructed Irom 128 frequency bins spaced at $100 \mathrm{~Hz}$. Of the $12.8 \mathrm{KHz}$ only $10 \mathrm{KHz}$ is displayed, $7 \mathrm{KHz}$ reserved for forward flow and $3 \mathrm{KHz}$ reserved for reversed flow. The FFI andyzer samples the (wo quadrature channels at a $12.8 \mathrm{KHz}$ range and provides spectra at a rate of 400 per second. Since each spectrum is composed of $25 \%$ new data and $75 \%$ old data, every fourth spectrum is independent from a statistical viewpoint. This method is appropriate, where the input signal is not stationary but continually varying with time. A hardcopy of the output of the spectral analysis is obtained on light-sensitive paper, where frequency is displayed versus time with the amplitudes as levels of grey.

Before the displaty of the signal, the signal is "normallized" in order to improve the signal-to-noise ratio. The principle of normalizing is based on increasing the highest amplitude of each analysis to a reference level with the same scaling factor applied to all other amplitudes (Cigure 8).

\section{Normalizing}

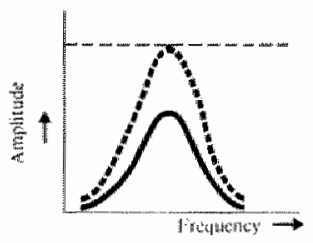

Figure 8 .

The principle of "wormalizing" is based on the increase of the highest amplitude to a reference level. Amplitude is displayed on the vertical, ind frequency on the horizontall axis.

Following normalizing only a certain amount of the signal is displayed, according to the "dynamic range" of the Doppler signal. A dynamic range of only $15 \mathrm{~dB}$ is chosen (figure 9), whenever a large dynamic range is chosen $(60 \mathrm{~dB})$ the chance exists that not only the desired Doppler signal but also noise will be displayed.

Dynamic Ringe

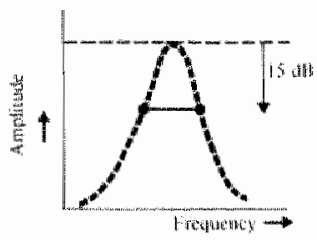

Figureg.

The "dynamic range" is measured from the relerence level. For cencestream information a dynamic range of $15 \mathrm{~dB}$ was chosen. Amplitude is displayed on the vertical, and trequency on the horizontal axis.

Although disturbed flow associated with high grade stenosis can be readily distinguished from normal flow by audible means ${ }^{139}$, flow disturbances resulting from lesser degrees of narrowing require more sensitive methods such as spectral analysis. The real-time FFT spectrum analyzer helps the technician performing the study to determine the frequency, direction and characteristics of the obtained signals. The quadrature outputs from the pulsed Doppler together with the corresponding B-mode images are fed to a Sony V02600 video tape recorder for permanent recording and analysis. 


\subsubsection{Patient examination method.}

The study is performed with the patient supine and the head supported to eliminate lateral movement. A water-soluble acoustic gel is used to couple the sound from the scan head to the skin surface. Blood vessels are identified by their characteristic B-mode images with prominent wall echos separated by a dark. sonolucent lumen (see figure 10). Calcified plaques are recognized as bright

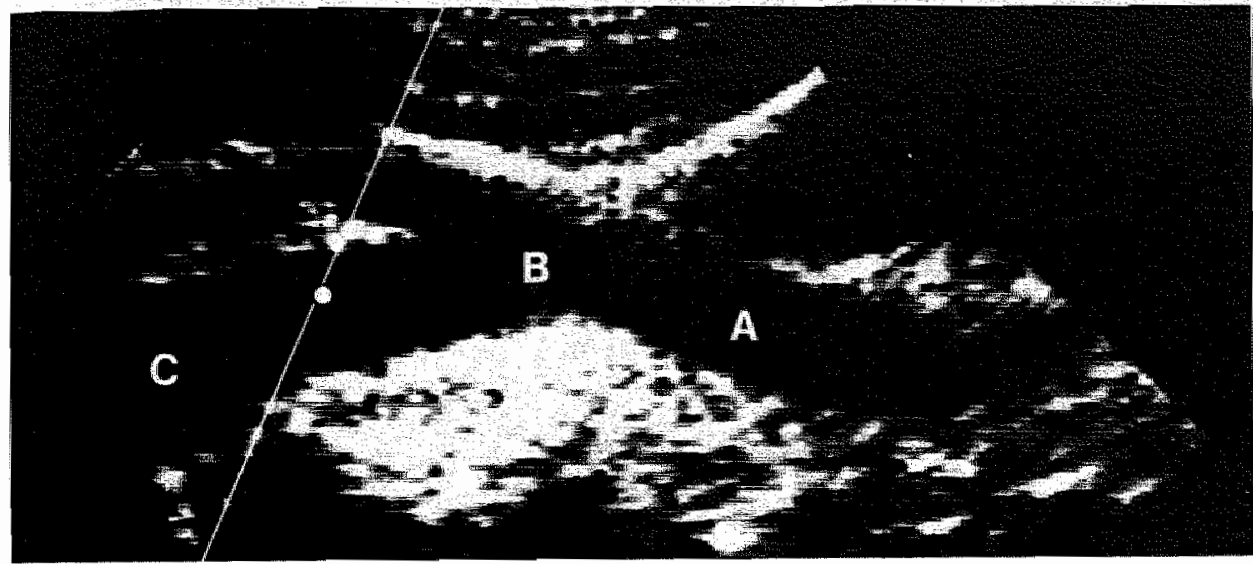

Figune 10,

B-mode image of a normal common carotid $(A)$, carotid bub (B), and internal carotid artery (C). The white line represents the direction of the pubed Doppler system. The white dot represents the postion of the Doppler sample wolume.

intraluminal echoes and regions of posterior acoustic shadowing (see figure II). The operator places the sample volume of the pulsed Doppler in the centersiream

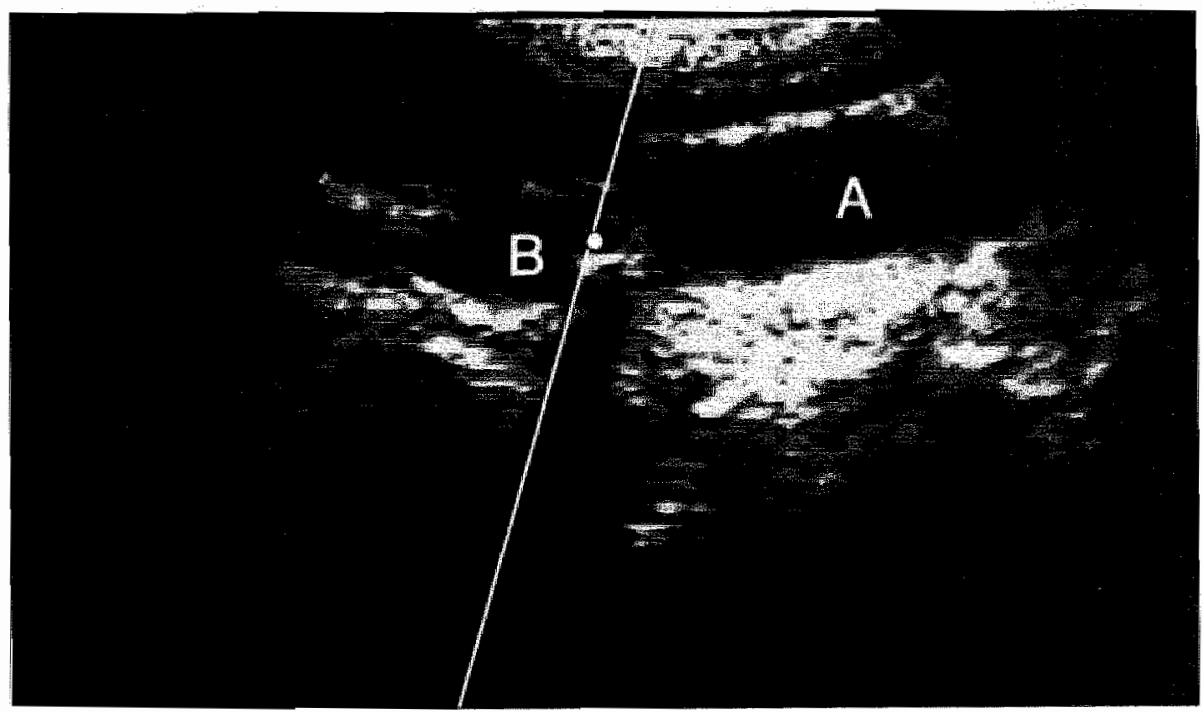

Figure 11 .

B-mode image of a stenotic proximal inemal carotsd artery. A is the common carotid. $B$ the internat carotid artery. Note the calcified plaque with the posterior acoustic shadowing in the proximal internal carotidartery. 
of the vessel and measures the angle of the Doppler beam relative to the axis of the vessel of interest. A Doppler angle of approximately 60 degrees provides a favorable return signal for further processing.

The Doppler sample volume is placed near the centerstream of the vessel in order to avoid detection of velocities and eddies close to the wall. These eddies would cause an increase of the spectal width even in normal arterial vessels. In order to obtain the most representative signal the operator monitors the amplitude versus frequency output from the FFT on an oscilloscope, the audible signal from the speaker and the screen of the real-time spectrum analyzer.

The examination begins low in the neck with the identification of the common carotid artery. Moving distally, the carotid bulb can be recognized by a typical audible signal. By scanning in various planes the bifurcation can often be visualized with the external carotid artery usually anteromedial to the internal carotid artery (see figure 12). The differentiation between the internal and external carotid artery is made by characteristic Doppler signals, noted as the sample volume moved through the scan plane. Flow in the common and internal carotid artery is normally quasi-steady and always well above zero during the entire pulse cycle due to the low resistance of the cerebrowascular bed. Flow in the external carotid artery, supplying a vascular bed with a relative high peripheral resistance (the face), either goes to zero or reverses in diastole. This difference in the characteristics of the velocity signals makes it possible to differentiate accurately between the intermall and external carotid artery.

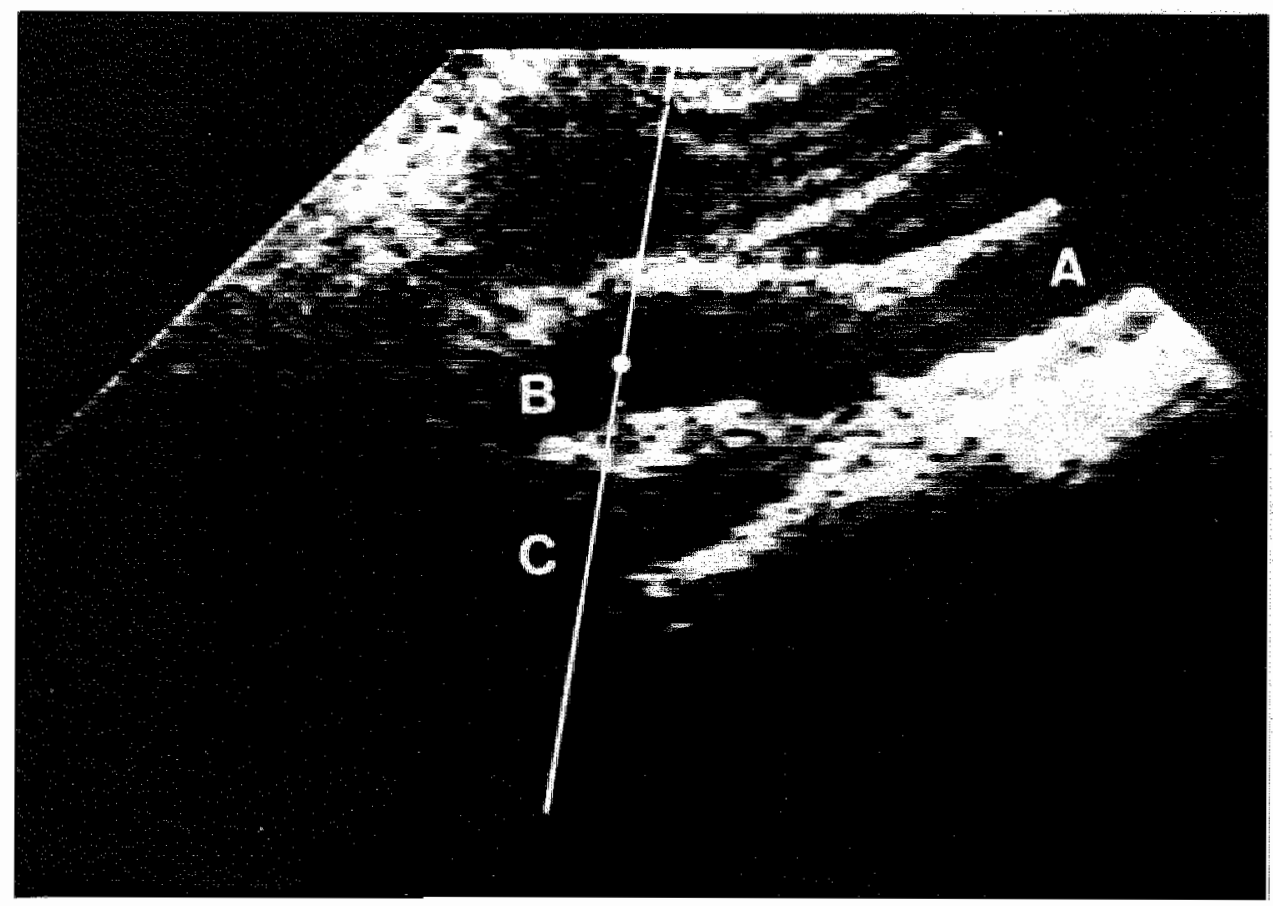

Figure 12.

B-mode image of anomal carotid bifurcation. A is the common carotid. B the external carotict, and $C$ the intermal carcotid artery. Note the jugtar wein above the common carond artery. 
When the common, internal and external carotid artery are localized, signals from the common carotid artery, the proximal internal carotid artery, the distal internal carotid artery and the external carotid artery are recorded on videotape for documentation and further analysis. The whole study of both carotid sides takes approximately 30 minutes in the hands of an experienced technician.

After the study is completed the quadrature information from the pulsed Doppler is analyzed using a Fast Fourier Transform spectrum analyzer and recorded on light sensitive paper. The hard copy displays frequency on the vertical axis versus time on the horizontal axis with the amplitudes expressed as levels of grey. Eight levels of grey are chosen for displaying the different amplitudes for routine studies. The frequency range is $10 \mathrm{KHz}$, where $7 \mathrm{KHz}$ is reserved for forward flow and $3 \mathrm{KHz}$ for reversed flow.

A normal Doppler spectrum consists of systolic peak frequency up to $4 \mathrm{KHz}$ and a narrow band of frequencies during systole, resulting in a clear region beneath the systolic peak (window). With minimal stenosis spectral changes occur as spectral broadening during the deceleration phase of systole. Further stenosis generally results in increased spectral broadening until the entire window beneath the systolic peak is filled, followed by an increase in peak systolic and diastolic frequencies ${ }^{90}$.

For the validation studies five categories of diameter reduction were used (see figure 13). The radiologists classified the biplanar contrast arteriograms with regard to the degree of involvement at the level of the bulb. They expressed the diameter reduction as a percentage of the estimated normal wall diameter to the nearest five percent (see figure 13). The unsubtracted arteriograms were used because the presence of calcification in the outer layers of the vessel wall in the region of the stenosis facilitated the estimation of the normal wall diameter. The radiologists reporting the diameter reductions were unaware of the results of the noninvasive tests, the technicians performing the noninvasive studies were unaware of the results of the contrast arteriograms.

\section{Diameter Reduction}

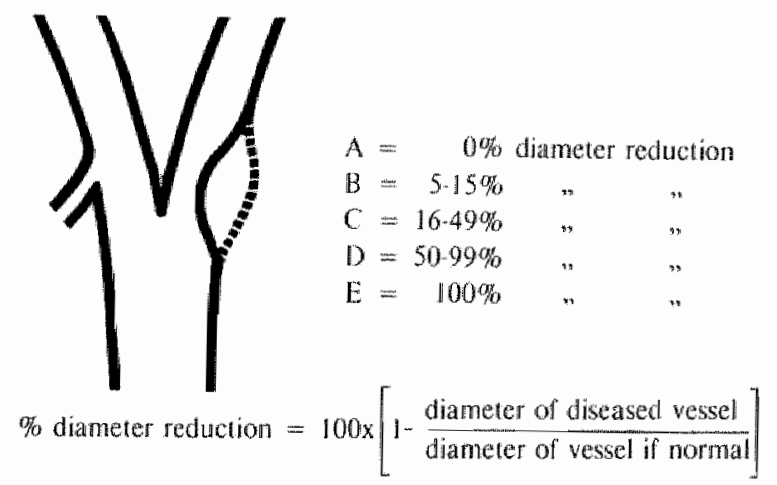

Figure 13 .

Arteriographic classification of the proximal internal carotid artery. 
The spectral changes used to define each category include:

(A) normal: systolic peak frequency up to $4 \mathrm{KHz}$ without spectral broadening; (B) 5-15\% diameter reduction: systolic peak frequency up to $4 \mathrm{KHz}$, spectral broadening in the deceleration phase of systole; (C) 16-49\% diameter reduction: systolic peak frequency up to $4 \mathrm{KHz}$, spectral broadening throughout systole; (D) $50-99 \%$ diameter reduction: systolic peak frequency above $4 \mathrm{KHz}$, spectral broadening throughout systole, increased frequencies in diastole; and (E) occlusion: no signal in the internal carotid artery, flow to zero during diastole in the common carotid artery.

It should be noted that in contrast to previous publications from our laboratory ${ }^{5,9,(6)}$, the arteriographic classification has been changed. In the new classification the "B" category has changed from diameter reduction less than $10 \%$ to 5-15\% diameter reduction. The " $\mathrm{C}$ " category changed from $10-49 \%$ to 16 $49 \%$ diameter reduction. There are two reasons for the new classification. In the first place from a clinical point of view a cutpoint at $15 \%$ diameter reduction seems to be more practical ${ }^{\text {tot }}$. Secondly, experience with the computer controlled pattern recognition program, as developed at the University of Washington, also demonstrates a cutpoint at $15 \%$ diameter reduction. Introducing the new classitication makes it possible to compare the spectral analysis hardcopy results with the pattern recognition computer results. 


\section{Chapter 5}

\section{Accuracy of Duplex scanning.}

\subsection{Introduction.}

The accuracy of the Mark V Duplex scanner combined with spectral analysis as compared with contrast arteriography will be presented and discussed in this chapter. Four separate studies form the basis of the data for this evaluation. "The first study was performed after the results with the Periperal Vascular Scanner III were analyzed". It became obvious that the accuracy of predicting a total occlusion of the internal carotid artery by the noninvasive test could be improved by paying more attention to the velocity pattern of the common carotid artery. In order to assess in an objective fashion the value of the velocily pattern of the common carotid artery as related to disease at the catrotid bifurcation, the velocity patterns of 156 patients who had undergone contrast arteriography were reviewed.

In the second study the accuracy of the Mark V Duplex scanner in combination with spectral analysis was evaluated. Multiplanar contrast arteriograns of 240 carotid sides were available for comparison. The purpose of this study was to review the accuracy of the noninvasive method and its potential applications for clinical research studies.

The spectral changes observed with early disease are often minimal and theretion difficult to separate from velocity patterns in normal carotid arteries. In order to "unmask" lesser degrees of stenoses, we considered to increase the blood flow to the brain by giving patients with minimal disease carbon dioxide. However before applying this method to patients, it was necessary to evaluate the changes in rormal subjects. The third study of this chapter was designed to evaluate the velocity pattern changes that occur when there is an increase of total cerchiat blood flow in normal subjects.

The fourth study concerned the evalluation of a parameter for accurate classification of severe carotid disease. The study was performed in order to estimate the degree of involvement in patients with stenoses within the $50.99 \%$ diameter reduction category.

\subsection{The role of common carotid artery velocity patterns in the} evaluation of carotid bifurcation disease.

\subsubsection{Introduction.}

Over the past three years the application of a prototype Duplex scanner has heen evaluated 24.5\%. 164 . With the development of the Fast Fourier Transform spectrum analyzer, which could be used to accept the directional signals from the pulsed Doppler, it became apparent that the velocity patterns recorded from lhe 
common carotid artery could be of additional use in detecting high grade stenoses or total occlusions of the internal catrotid artery.

Fell et al. ${ }^{50}$ found in their study, using the periperal vascular Duplex scanner III, an agreement between contrast arteriography and the noninvasive test for the total acclusion category of $70 \%$. Of the 46 arteriographic occluded internal carotid arteries only 32 were predicted correctly by the Duplex scanner. Since the differentiation between a high grade stenosis and a total occlusion of the internal carotid artery is of great clinical importance, inaccurately classifying total occlusions was of great concern.

A study of the velocity patterns of the common carotid artery using the Duplex scanner was performed by Bodily et al. ${ }^{31}$. They observed flow to zero during late systole or diastole in 33 of the 34 sides with internal carotid occlusion. It should be stressed however that, whenever flow to zero is used to characterize the velocity pattern of the common carotid artery, the angle between the Doppler beam and the axis of the vessel should be optimal. In order to demonstrate the changes in the velocity patterns that occur with different Doppler angles, hardcopy of the velocity pattern from the common carotid artery were recordered over a range of 45 degrees to 90 degrees in steps of 15 degrees. (see figure 14-17). In our studies we routinely establish a Doppler angle of 60 degrees to obtain a favorable return signal for further processing.

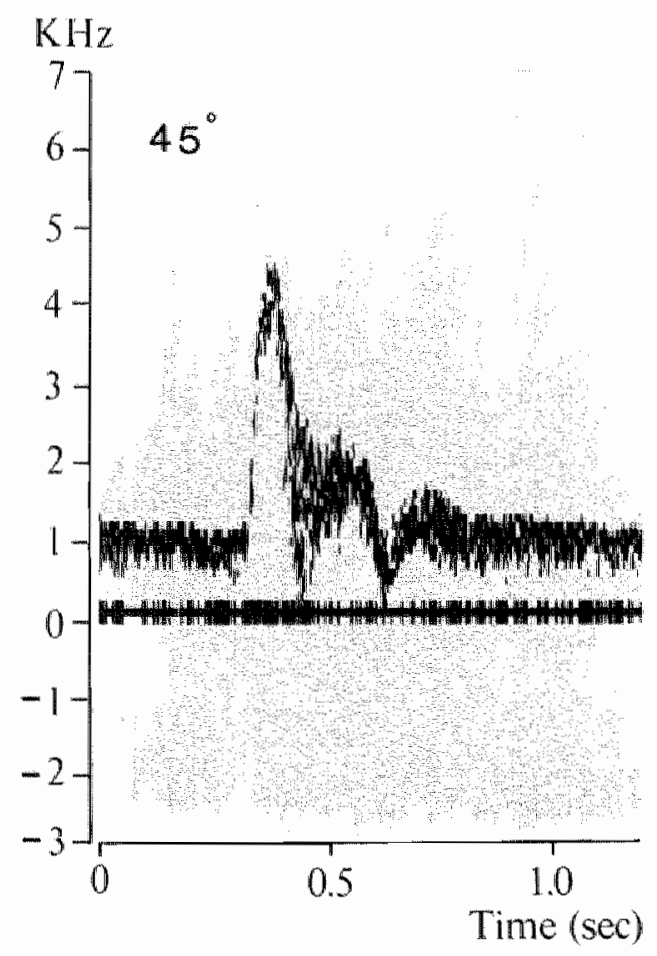

Figure 14.

Hardcopy of the velocity pattern of a normal common carotid artery. The angle between the Doppler beam and axis of the artery was $45^{\circ}$. Note the frequencies above zero during diasto. le. 

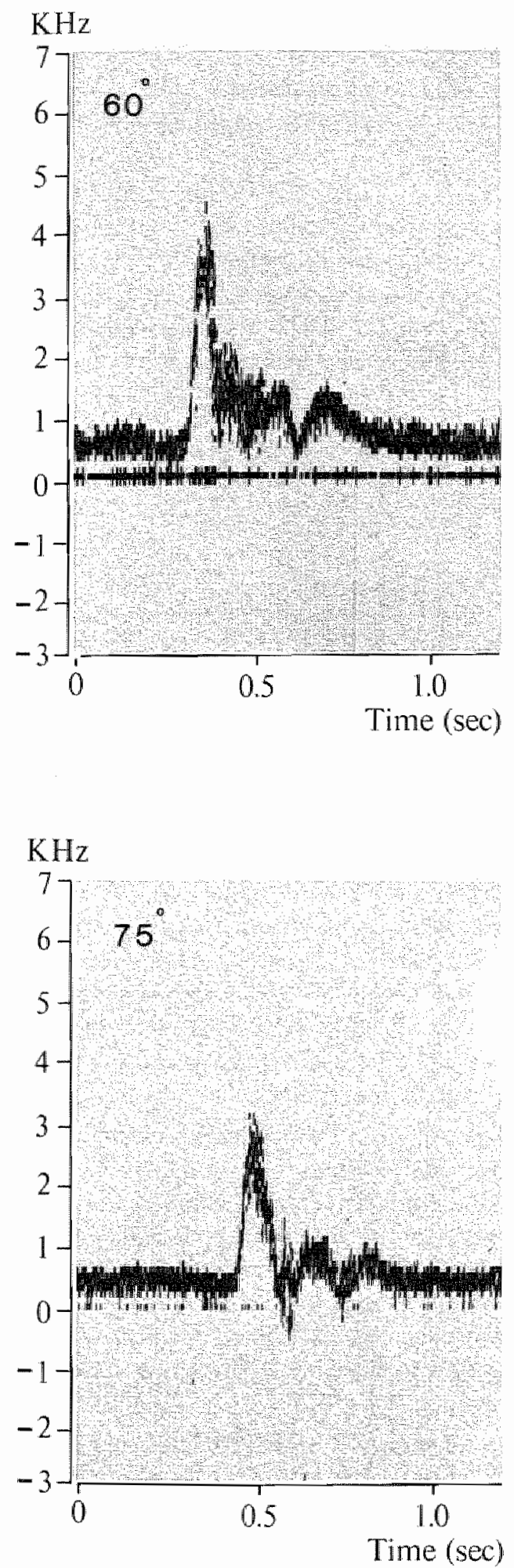

Figure 15.

Hardeopy of the wotocity paldern of a mormat common carotid artery. The angle beween the Doppler beam and the axis of the artery was $60^{\circ}$. The frequencies during dastole remain above 20 . 10 .
Fingute 16

Hardcopy of the velocity pattern of a normal common canotid atcry. The angle between the Doppler beam and the axis of the antery was 75. Peak systolic frequencies are decreased. the frequencies dering diastole reach the zero linen. 


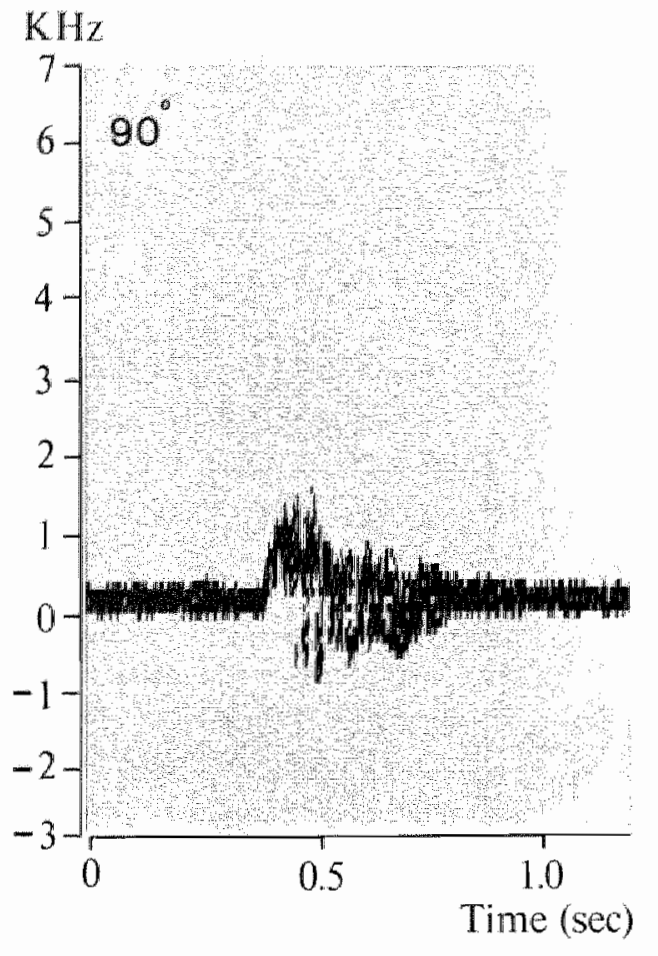

Figure 17

Hardicopy of the velocity pattern of a normal common carotid artery. The angle between the Doppler beam and the axis of the vessel was $90^{\circ}$. The velocity pattem has lost all characteristics of a common carotid antery.

Flow in the common carotid artery is normally quasi-steady and always well above zero during the entire pulse cycle due to the low resistance of the cerebrovascular bed ${ }^{120.122,148 .}$. At the bifurcation, flow is separated to supply two vascular beds each with different peripherall resistance. The internal carotid artery supplies a low resistance vascular bed, allowing marked forward flow during diastole resulting in a flow above zero at all times in the pulse cycle. The velocity pattern of the common carotid artery is dominated by the low resistance of the ipsilateral hemisphere giving a llow pattern which is similar to that observed in the internal carotid artery. "This latter velocity pattern can be influenced by changes in peripheral resistance ${ }^{147,130}$, and central hemodynamic changes ${ }^{\text {las. }}$.

Significant occlusive disease of the internal carotid artery which increases the resistance to llow, may be detected by changes in the common carotid artery velocities patterns 3.134 . A total occlusion of the internal carotid artery produces a flow pattern in the common carotid attery resembling that in the external carotid artery; i.e. the flow will go to zero during diastole and even reverse in some instances. Thus, when no velocity signal is found in the region of the internal carotid artery and a total occlusion of the internal carotid artery is considered, the diagnosis can be conlimmed by the finding of flow to zero in the common carotid artery.

Flow to zero in diastole with reversal of flow during late systole can also occur in the velocity pattern of a common carotid artery of patients with aortic valve disease 146 . In this case, it is expected to be found bilaterally.

To assess in an objective fashion the frequency of flow to zero, as related to 
disease in the carotid bifurcation, the velocity patterns of the common carotid artery of 156 patients who had undergone biplanar contrast arteriography were reviewed. Data was obtained with both the prototype PV III Duplex scanner and the Mark V Duplex scanner.

\subsubsection{Results.}

The velocity waveform from a common carotid artery without disease in the internal carotid artery is shown in figure 18 . Flow is well above zero al all points in the pulse cycle. The velocity waveform from a common carotid artery with a total occlusion of the internal carotid artery is shown in figure 19. Flow goes to zero during diastole with flow reversal during late systole.

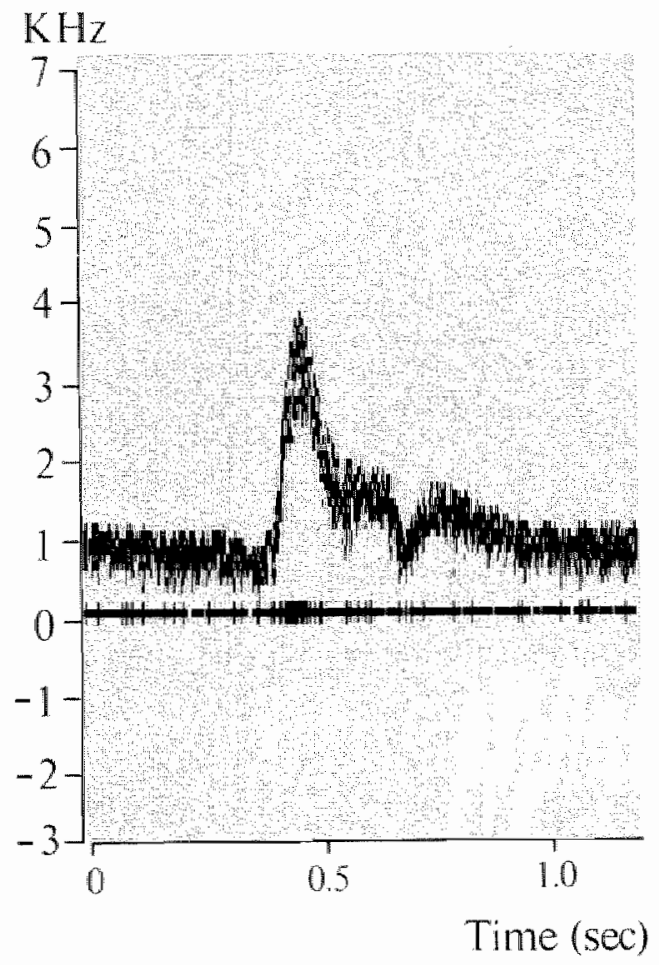

Figure 18.

Hardcopy of the velocity patern of a common carotid artery whoul disedse in the intemal carotid artery. Note the freguencies abowe zero during diastole. 


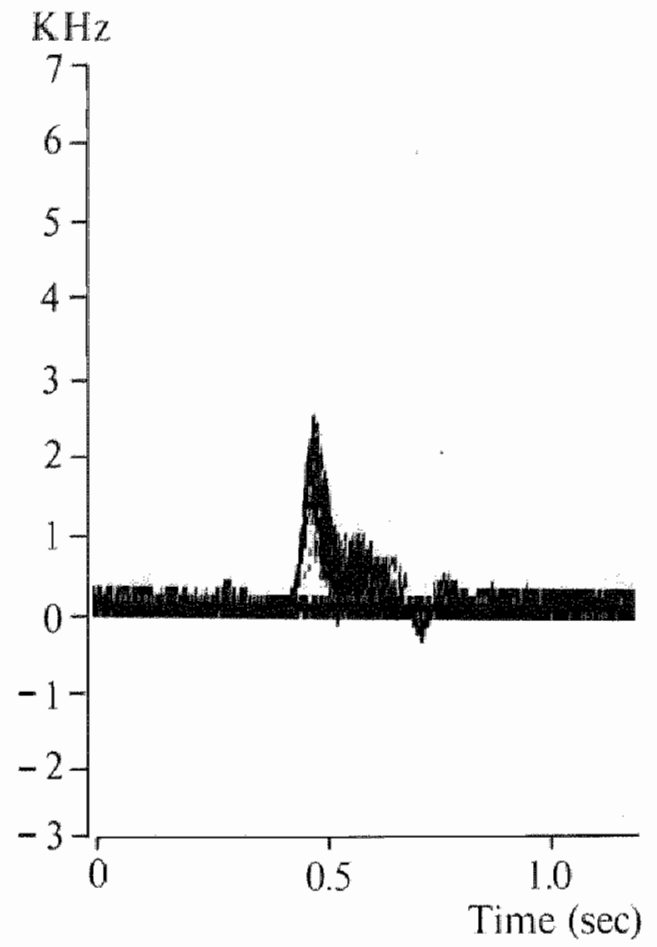

Figure 19.

Hardoopy of the velocity pattern of a common carotid artery with an occlusion of the internal carotid artery. Note the frequencies reaching zero during diastolc.

The distribution of the degree of involvement of the internal carotid artery as classilied by the velocily patterns and confirmed by contrast arteriography is shown in Table 1. A total occlusion of the internal carotid artery was found in $18 \%$ $(56 / 312)$, a $50-69 \%$ diameter reduction in $37 \%(116 / 312)$, a $16-49 \%$ diameter reduction in $34 \%(107 / 312)$, a $5-15 \%$ diameter reduction in $8 \%(23 / 312)$, and a normal internal carotid antery in $3 \%$ (10/312).

Tiable I.

Arteriographically estimatled pereent diameter reduction of the intemal carotid artery and flow to zero in the common carblid artery.

\begin{tabular}{lc|ccc}
$\begin{array}{l}\text { Internal Carotid Artery } \\
\text { Category }\end{array}$ & Sides & $\begin{array}{c}\text { Common Carotid Artery } \\
\text { Unilateral Flow } \\
\text { to Zero(Sides) }\end{array}$ & $\begin{array}{c}\text { Bilateral Flow } \\
\text { to Zero (Sides) }\end{array}$ & $\begin{array}{c}\text { Total Sides With } \\
\text { Flow to Zero }\end{array}$ \\
\hline A. Normal & 10 & 0 & 0 & 0 \\
B. $5-15 \%$ & 23 & 0 & 3 & 3 \\
C. $16-49 \%$ & 107 & 0 & 9 & 9 \\
D. $50-99 \%$ & 116 & 15 & 11 & 26 \\
E. Occlusion & 56 & 39 & 13 & 52 \\
\hline Total & 312 & 44 & 36 & 90 \\
\hline
\end{tabular}


As noted in Table $I$, flow to zero was found in the common carotid artery in 90 of the 312 sides ( $29 \%$ ). The highest percentage was found in cases of total occlusion of the internal carotid artery, 52 of $56(93 \%)$. Of these 52 sides, flow to zero was found unilaterally in 39 and bilaterally in 13 sides. The 13 sides with bilateral flow to zero were found in eight patients; five patients had bilateral occlusions of the internal carotid artery ( 10 sides). and three patients had a contralateral $50-99 \%$ diameter reduction ( 3 sides).

With a $50-99 \%$ diameter reduction of the proximat internal carotid antery (D category), flow went to zero in $22 \%(26 / 116)$. In those cases with stenoses of less than $50 \%$ diameter reduction ( $\mathrm{B}$ and $\mathrm{C}$ category) flow went to zero on twelve sides, in each instance flow to zero was found bilaterally.

Further breakdown of the 50-99\% diameter reduction group is shown in Table II. The Table shows three groups of internal carotid arteries: (1) total occlusions. (2) tight stenoses, with a diameter reduction of $90-99 \%$, and (3) stenoses with diameter reductions less than $90 \%$ and normal internal carotid arteries. In the tight stenosis group (90-99\% diameter reduction) flow to zero was found in $52 \%$ (11/21). In nine catses the finding was unilateral, in two cases it was a bilateral finding. In one of these two cases the contralateral carotid artery was occluded, in the second case, there was a diameter reduction of less than $90 \%$ in the contralateral internal carotid artery.

Table II.

Arteriographically estimated percent diameter reduction of the internal carotid artery and flow to ze ro in the common carotid artery.

\begin{tabular}{|c|c|c|c|c|}
\hline \multicolumn{2}{|c|}{ Internal Carotid Artery } & \multicolumn{3}{|c|}{ Common Carotid Artery } \\
\hline Category & Sides & $\begin{array}{l}\text { Unilateral Flow } \\
\text { To Zero(Sides) }\end{array}$ & $\begin{array}{l}\text { Bilateral Flow } \\
\text { ToZero(Sides) }\end{array}$ & $\begin{array}{l}\text { Total Sides With } \\
\text { Flow ToZZero }\end{array}$ \\
\hline 1. Occlusion & 56 & 39 & 13 & 52 \\
\hline $\begin{array}{l}\text { 2. Tight Stenosis ( } 90 \\
-99 \% \text { Diameter } \\
\text { Reduction) }\end{array}$ & 21 & 9 & 2 & 11 \\
\hline $\begin{array}{l}\text { 3. Stenosis Less than } \\
90 \% \text { Diameter } \\
\text { Reduction }\end{array}$ & 235 & 6 & 21 & 27 \\
\hline Total & 312 & 44 & 36 & 90 \\
\hline
\end{tabular}

If the total occlusion and tight stenosis groups (Table II) are excluded from the analysis, flow to zero in the common carotid artery was found in $11 \%$ ( 27 sides). Of these 27 sides it were noted to be bilateral in 21 instances $(78 \%)$. 


\subsubsection{Discussion.}

Flow to zero in late systole or during diastole in the common carotid artery can occur as a reflection of central hemodynamic changes the or a a reflection of increased peripherall resistance due to atherosclerotic changes in the internal catrotidartery ${ }^{3 !}$.

In the present data the finding of flow to zero was observed in $93 \%$ (52/56) of the cases of an internall carotid artery occlusion. In these instances the phenomenon can be explained as a result of increased peripheral resistance. An increased resistance however can also occur when there is a tight stenosis of the proximal internal carotid artery. In the present study, flow to zero in the common carotid artery with a light stenosis in the internal carotid artery was found in $52 \%(11 / 21)$. In this situation, in contrast to a total occlusion, a high velocity signal should be found in the region of the stenosis. which is usually at the level of the proximal inconal carotid artery.

The finding of this high velocity signal is very important, since the clinical consequences of the diagnosis of a tight stenosis are different from that of a total occlusion. A very tight stenosis of the proximal internal carotid artery in a patient with focal neurological symptoms is considered amenable to carotid endaterectomy, whereas the patient with a total occlusion is not considered to be candidate for this procedure. "This stresses the importance of searching for velocity signals of the internal carotid despite the finding of unilateral flow to zero in the common carotid artery.

Whenever flow to zero is found bilaterally in the common carotid artery during the study, the technician should realize that this may be secondary to bilateral peripheral resistance changes or to central hemodynamic changes. For example. aortic valve insulficiency will cause bilateral flow to zero in diastole with reversal of flow during late systole. Under these circumstances, the characteristics of the velocity signals from the internal carotid artery are used to distinguish a central catuse from bilateral internal carotid artery pathology. In the present data this is demonstrated by the fact that the finding of flow to zero in the common carotid arlery with minimal disease in the internal carolid artery (category $B$ and $C$ ) was bilateral in all instances, suggesting that the finding was due to central pathology.

Flow to zero in the common carotid artery was not found in combination with a total occlusion of the proximal internal carotid artery in four sides. The reason for this linding is not clear, an explanation could be that due to the excellent collateral system viat the external carotid artery the peripheral resistance was not markedly incrersed.

Billateral flow to zero was found in the common carotid artery with an occlusion of the internal carotid antery in 13 sides, the billateral finding was explained in ten sides by ablateral occlusion of the internal carotid artery and in one case the contralateral internal carotid artery was found to have a tight stenosis (90-99\% diameter reduction). In the remaining two sides there was no peripherat resistance change to explain the finding.

In conclusion, there is evidence from the data in this study that there are three groups of patients with flow to zero in the common carotid artery: (1) patients 
with billateral flow to zero in the common carotid artery not related to occlusive disease of the internal carotid artery; (2) patients with flow to zero in the common carotid artery with tight stenoses ( $90-99 \%$ diameter reduction) of the internal carotid artery; and (3) patients with flow to zero in the common carotid artery with total occlusions of the internal carotid artery.

Obtaining the velocity patterns of the common carotid artery should be a part of the total moninvasive study. The technician, performing the study, should understand the reasons for changes occuring in the velocity pattern of the common carotid artery. As will be demonstratted in section 5.3., the appreciation of the characteristics of the velocity pattern of the common carotid artery has considerably improved the accuracy of the Duplex scanner combined with spectral analysis in predicting high grade lesions and total occlusions of the internal carotid artery.

\subsection{The accuracy of ultrasonic Duplex scanning as compared with contrast arteriography}

\subsubsection{Introduction.}

During the past decade. a variety of indirect noninvasive tesis have been developed to assess carotid bifurcation arteriosclerosis. The most commonly used included periorbital Doppler 13.32. 11 and plethysmographic methods 50, ow, 9.4 which are theoretically capable of detecting those lesions which produce a reduction in pressure and flow. However, these methods cannot distinguish between a high grade stenosis and at total occlusion of the internal carotid antery. Furthermore they cannot differentiate between normal and minimal disease (non pressure or flow reducing lesions). It is now known that disease of the carotid bifurcation which may produce transient ischemic attacks and strokes can vary from minimal lesions to total occlusion ${ }^{160}$. It is, therefore, necessary to utilize methods of noninvasive lesting which can identify both normal vessels and hose whth the entire spectrum of involvement.

The approach taken at the University of Washington has been to develop an ultrasonic system which not only permits imaging of the bifureation, but also altempts to identify flow changes at that level which occur secondary to the presence of atherosclerosis. This has led to the development of the ultrasonic Duplex scanner which permits such studies to be made ${ }^{132.133}$. Most recently the results of the prototype PV III Duplex scanner were reported by Fell et al. ${ }^{5}$. In this study 135 patients underwent contrast arteriography, giving 270 carotid bifurcations available for comparison. The agreement between contrast arteriography and ultrasonic Duplex scanning combined with spectral analysis of this study is shown in Table III. For a normall bifurcation the agreement was $35.5 \%(4 / 11)$, for a diameter reduction less than $10 \%$ the agreement was $45 \%$ $(9 / 20)$, for a $10-49 \%$ diameter reduction the agreement was $58 \%(52 / 90)$, for a 50 $99 \%$ diameter reduction the agreement was $81.5 \%(84 / 103)$, and for a total occlusion $70 \%(32 / 46)$. This study demonstrates that, although far from perfect, the method of Duplex scanning combined with spectral analysis has the potential 
10 predict the extent of involvement present, warying from plaques not producing diameter reductions to those with a total occlusion of the internal carotid artery. In January 1980, the PV III Duplex scanner was replaced by the commercially available Mark V Duplex scanner. The improvements made were in large part the results of experience gained with the prototype PV III scanner. The accuracy of the Mark V Duplex scanner is the subject of this section.

Table ll.

Correlation of PV III Duplex results and arteriographic results for the internal carotid artery.

Duplex (PV IIT)

\begin{tabular}{r|c|c|c|c|c|c} 
Arteriography & $0 \%$ & $<10 \%$ & $10-49 \%$ & $50-99 \%$ & $100 \%$ & \\
\hline $0 \%$ & 4 & 3 & 4 & & & 11 \\
\hline$<10 \%$ & 3 & 9 & 8 & & & 20 \\
\hline $10-49 \%$ & 4 & 12 & 52 & 20 & 2 & 90 \\
\hline $50-99 \%$ & & 3 & 10 & 84 & 6 & 103 \\
\hline $100 \%$ & & & 2 & 12 & 32 & 46 \\
\hline & 11 & 27 & 76 & 116 & 40 & 270
\end{tabular}

\subsubsection{Methods and Materials.}

The Mark V Duplex scanner used for this study is described in section 4.3. The system combines real-lime B-mode imaging (see 4.1.4.) with a pulsed Doppler device (see 4.1.3.). The B-mode images are used to identify the carotid arteries and to visualize callified plaques. When the B-mode image is "frozen" (seefigure 20 ), one of the crystals is used as a pulsed Doppler. This permits the use of a pulse repetition frequency of $19.2 \mathrm{KHz}$, which allows a Doppler frequency response up to $9.6 \mathrm{KHz}$ belore aliasing occurs (see 4.1.3.).

In addition to the audible interpretation, the quadrature outputs of the Doppler are sent to an on-line spectrum analyzer employing a digital fast Fourier transform analysis. The spectrum analyzer (Honeywell prototype FFT Spectrum Analyzer) provides 400 spectra per second with a frequency display range of $10 \mathrm{KHz}, 7 \mathrm{KHz}$ are reserved for forward and $3 \mathrm{KHz}$ for reverse velocity components (see 4.3.3.). The characteristics of the Doppler spectrum have been described previously (see 4.3.4.1. Hardcopy output ol the spectral analysis is obtained on light sensitive paper. where frequencies are displayed vertically as a function of time with the amplitudes of the backscattered signal expressed in levels of gray (see 4.3.4.). 


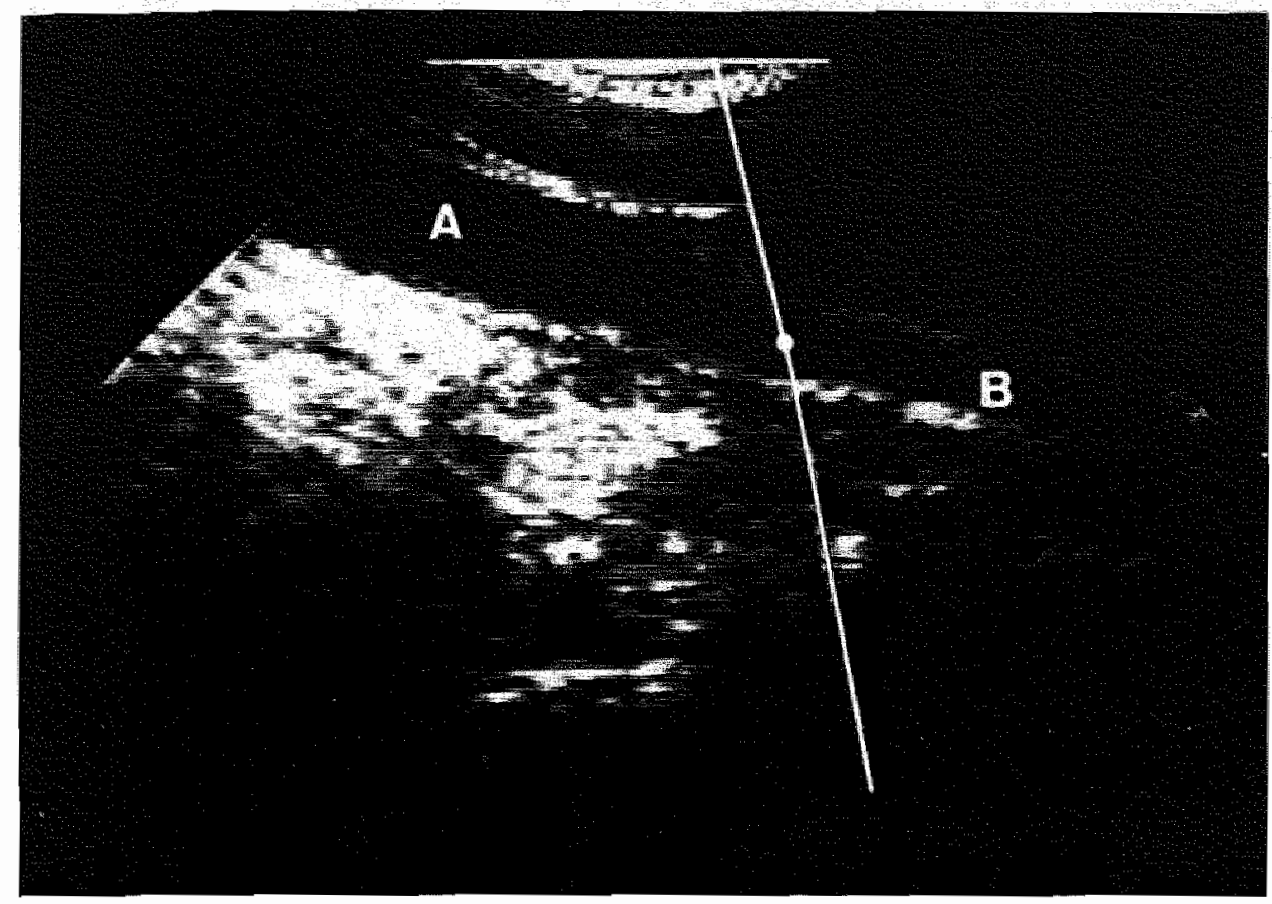

Figure 20.

B-mode image of a normal common carotid (A) and internal carotid artery (B). With the B-mode image "frozen", one of the crystals is used as a pulsed Doppler. The white dot is the location oll the sample volume of the Doppler system.

From January 1980 till November 1980 the medium focus scanthead was used, in this period 500 patients were referted to the laboratory for a noninvasive evaluation of their carotid arteries. Of these 500 patients 85 (169 sides) underwent multiplanar contrast arteriography. Beween November 1980 and May 1981250 patients were evaluated using the short focus scan head. Of these 250 patients 39 (77 sides) underwent multiplanar contrast arteriography.

The main difference between the two scan heads is, that the medium focus scan head employs $9.5 \mathrm{~mm}$. diameter transducers focused at $4 \mathrm{~cm}$ while the short focus scan head employs $6 \mathrm{~mm}$. diameter transducers locused at $2 \mathrm{~cm}$. (see 4.3.2.). Calculations of the sample volume size for the medium focus scan head using 20 $\mathrm{dB}$ measurements and assuming an oblate spheroid model at a range of $2 \mathrm{~cm}$. is 24 num ${ }^{3}$. the sample volume size for the short focus scan head using the same measurements at a range of $2 \mathrm{~cm}$ is $3 \mathrm{~mm}^{2}$ ( $\sec 4.3 .2$ ),

The radiologists reading the multiplanar contrast arteriograms were unaware of the results of the noninvasive study. The extent of disease of the proximal internal carotid was expressed as a percent diameter reduction of the normal wall diameter at the level of the stenosis (see 4.3.4.).

The arteriographic criteriu and velocity pattern characteristics used to categorize the extent of internal carotid artery disease are shown in table IV. It should be noted that in contrast to previous reports from the same institution, the B category 
Tablè IV.

Arteriographic and spectral analysis criteria for the classification of the internal carotid antery.

\section{Categories of Internal}

Carotid Artery

\begin{tabular}{|c|c|c|}
\hline Category & Arteriographic Criteria & Velocity Pattern Criteria \\
\hline A. & $\begin{array}{l}\text { Normal } \\
(0 \% \text { diameter reduction })\end{array}$ & $\begin{array}{l}\text { Systolic peak frequency up to } \\
4 \mathrm{KHz} \text {, no spectral broadening. }\end{array}$ \\
\hline B. & $5-15 \%$ diameter reduction & $\begin{array}{l}\text { Systolic peak frequency up to } \\
4 \mathrm{KHz} \text {, spectral broadening in } \\
\text { deceleration phase of systole. }\end{array}$ \\
\hline C. & $16-49 \%$ diameter reduction & $\begin{array}{l}\text { Systolic peak frequency up to } \\
4 \mathrm{KHz} \text {, spectral broadening } \\
\text { throughout systole. }\end{array}$ \\
\hline D. & $50-99 \%$ diameter reduction & $\begin{array}{l}\text { Systolic peak frequency above } \\
4 \mathrm{KHz} \text {, spectral broadening } \\
\text { throughout systole, increased } \\
\text { frequencies in diastole. }\end{array}$ \\
\hline E. & $\begin{array}{l}\text { Occlusion } \\
\text { (100\% diameter reduction) }\end{array}$ & $\begin{array}{l}\text { No signal, flow to zero } \\
\text { during diastole in common } \\
\text { carotid artery. }\end{array}$ \\
\hline
\end{tabular}

contains 5-15\% diameter reductions. One of the reasons for the new classification. as has been described previously (see 4.3.4.), was that the computer pattern recognition program employs a $5-15 \%$ diameter reduction group ${ }^{73}$.

Although the main emphasis in predicting extracranial carotid artery disease has been focused on the internal carotid artery, there are situations in which predicting the extent of external carotid artery disease can be of clinical importance. Thus it is important to have a classification scheme for the external carotid artery as well. For clinical purposes it is only necessary to differentiate between a normal external carotid artery, a non hemodynamic significant stenosis, a hemodynamic signilicant stenosis and a total occlusion. The arteriographic criteria and velocity pattern characteristics used to categorize the extent of external carotid artery disease are shown in table $\mathrm{V}$. 
Table $V$.

Arteriographic and spectral analysis criteria for the classification of the external carotid artery.

\section{Categories of External}

Carotid Artery

\begin{tabular}{|c|c|c|}
\hline Category & Arteriographic Criteria & Velocity Pattern Criteria \\
\hline 1. & Normal. & $\begin{array}{l}\text { Systolic peak frequency up } \\
\text { to } 4 \mathrm{KHz} \text {, no spectral } \\
\text { broadening. }\end{array}$ \\
\hline 2. & $5-49 \%$ reduction. & $\begin{array}{l}\text { Systolic peak frequency up to } \\
4 \mathrm{KHz}, \text { spectral broade- } \\
\text { ning in systole. }\end{array}$ \\
\hline 3. & $50-99 \%$ diameter reduction. & $\begin{array}{l}\text { Systolic peak frequency above } \\
4 \mathrm{KHz} \text {, marked spectral } \\
\text { broadening. }\end{array}$ \\
\hline 4. & Occlusion. & No signal. \\
\hline
\end{tabular}

\subsubsection{Results.}

Internal carotid artery. Hardcopy output of the spectrum analyzer from the 5 categories of the internal carotid artery are shown in figure 21-25. Note that the velocity waveform displayed in the total occlusion category is a common carotid artery waveform with flow to zero during diastole (see 5.2.3.). 

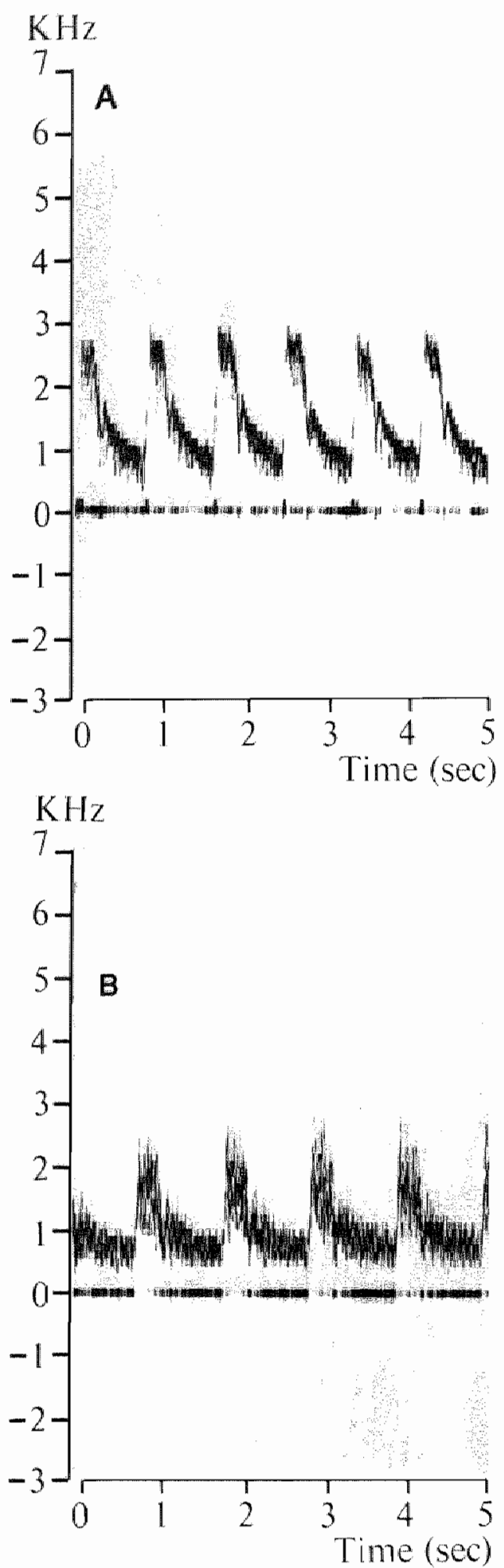

Figure 21

Hardcopy of a normal internal carotid artery signal. Frequency is displayed on the vertical and time on the horizontal axis, with the amplitude as level of grey. There is no spectral broadening during systolc. (clean area beneat th the systolic peak).
Figure 22.

Hardeopy of a $5-15 \%$ dianeter reduction of the internal carotid artery. There is specen ral broadening in the deceleration phase of systoin. 


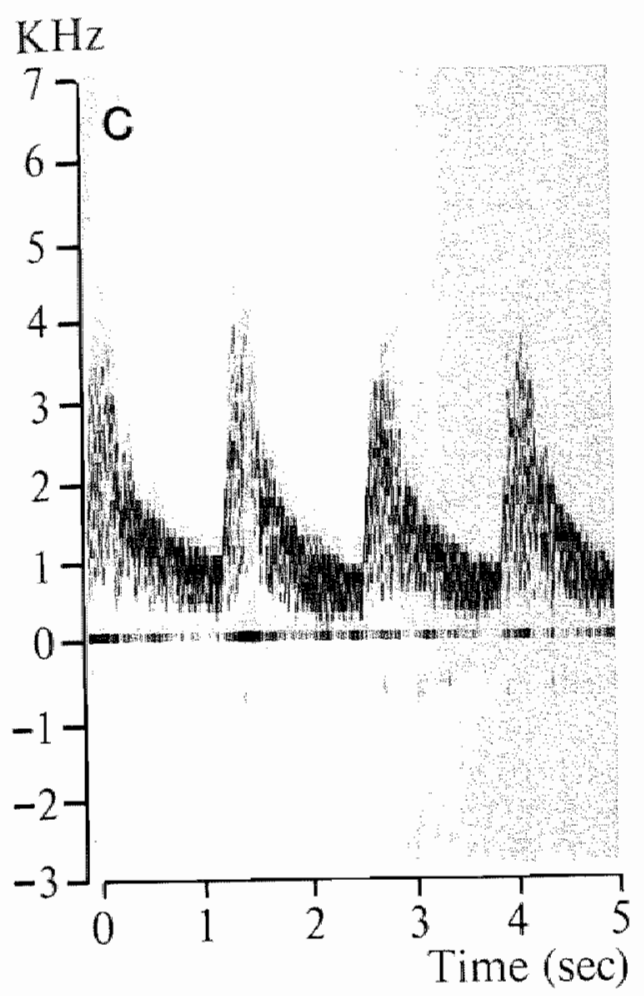

$\mathrm{KHz}$

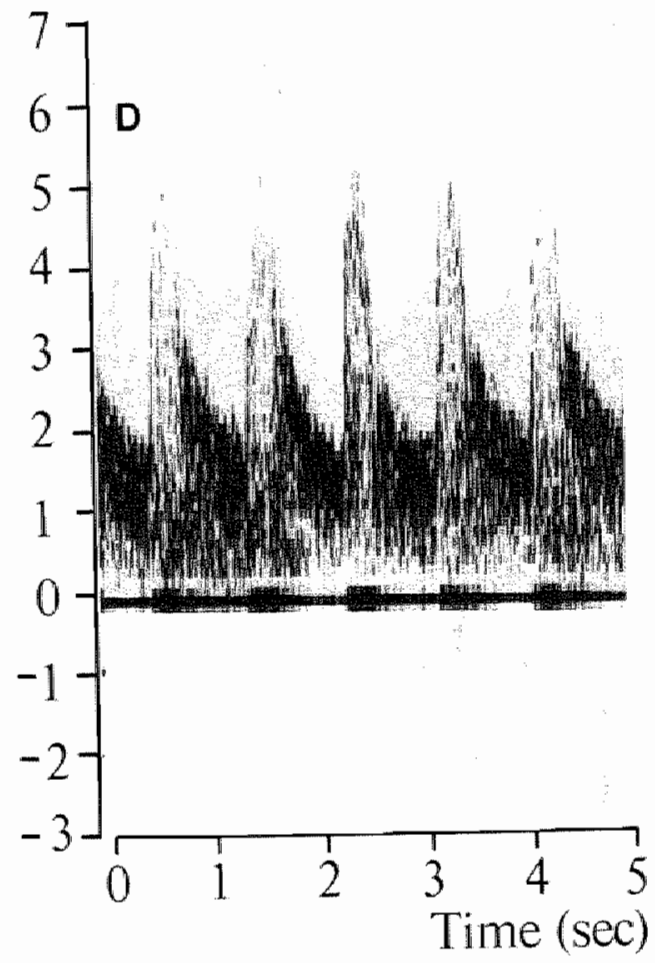

Figure 23.

Hardcopy of a $16-49 \%$ diameter reduction of the internal carotid artery. There is spectrat broadening throughout the entire pulse cycte.

\section{Figure 24.}

Hardcopy of a $50-90 \%$ diameler reduction of the internal canotid areery. Thare is marked increase in peak systolic and diastolic frequencies, associated with spectral broadening. 


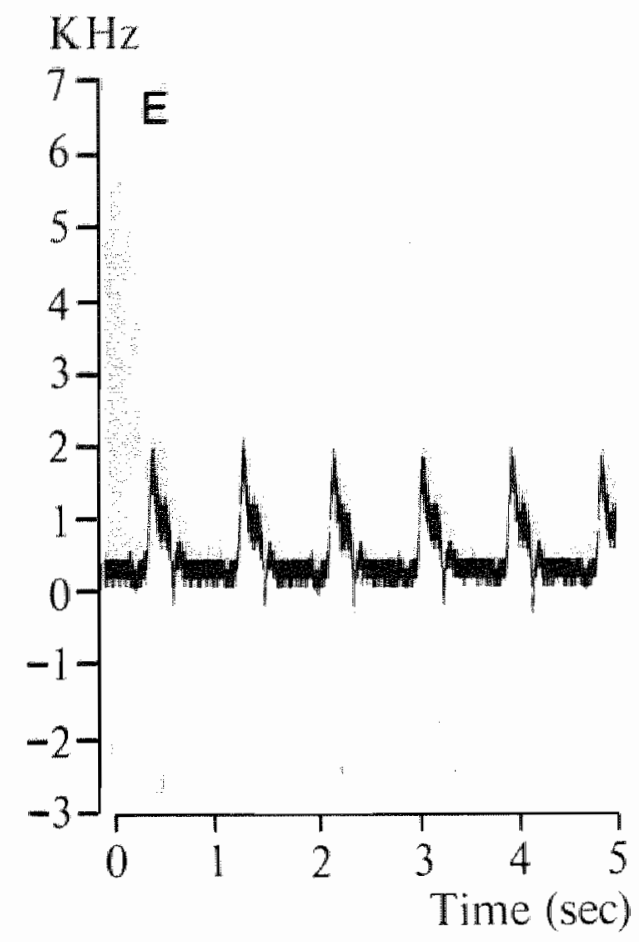

Figure 25 .

Hardoopy of a common carotid artery signal with a total occlusion of the internal carotid artery. Note the fllow to zero during diastole.

The resulis of the ultasonic Duplex scanner compared to the results of multiplanar contrast arteriography for the internal carotid artery using the medium focus scan head are shown in Table VI. Regarding the contrast arteriographic data as "gold standard", 7\% (12/169) of the internal carotid arteries

Table VI.

Correlation of Duplex results and arteriographic results for the internal carotid antery, using the medium focus scanhioad.

\section{Medium Focus Scanhead \\ Duplex}

\begin{tabular}{c|c|c|c|c|c|c} 
Arteriography & $0 \%$ & $5-15 \%$ & $16-49 \%$ & $50-99 \%$ & $100 \%$ & \\
\hline $0 \%$ & 1 & 6 & 4 & 1 & & 12 \\
\hline $5-15 \%$ & 1 & 19 & 15 & 2 & & 37 \\
\hline $16-49 \%$ & 2 & 10 & 24 & 5 & & 41 \\
\hline $50-99 \%$ & & 1 & 2 & 61 & 1 & 65 \\
\hline $100 \%$ & & & & 1 & 13 & 14 \\
\hline 4 & 36 & 45 & 70 & 14 & 169
\end{tabular}


were classified as normal ( $0 \%$ diameter reduction), $22 \%(37 / 169)$ had at $5-15 \%$ diameter reduction, $24 \%(41 / 169)$ had a $16-49 \%$ diameter reduction, $39 \%(65 /$ $169)$ had a $50-99 \%$ diameter reduction. and $8 \%(14 / 169)$ had a total occlusion (100\% diameter reduction).

Out of the twelve arteriographic normal carotid arteries, there was only agreement with the Duplex data in one, hence eleven sides were called diseased. In the 5-15\% diameter reduction category there was agreement in $51 \%(19 / 37)$. one side was undercalled and 17 sides were overcalled. In the 16-49\% diameter reduction category the agreement was $59 \%$ (24/41), twelve sides were undercalled and five sides were overcalled by the Duplex method. In the $50-99 \%$ diameter reduction category the agreement between the arteriographic data and Duplex data was $94 \%$ (61/65), three sides were undercalled and one side was overcalled by the Duplex method. In the total occlusion category ( $100 \%$ diameter reduction) the agreement with the Duplex data was $93 \%$ (13/14), one side was undercalled. The distribution of internal carotid artery disease as classified by contrast arteriography and studied with the short focus scan head is shown in table VII. Regarding the contrast arteriographic data as "gold standard", 10\% (8/77) were classified as normal ( $0 \%$ diameter reduction), 17\% (13/77) were classified as a 5 $15 \%$ diameter reduction, $30 \%(23 / 77)$ were classified as a $16-49 \%$ diameter reduction, $36 \%(28 / 77)$ were classified as a $50-99 \%$ diameter reduction, and $7 \%$ $(5 / 77)$ were classified as total occlusion ( $100 \%$ diameter reduction).

Table VII.

Correlation of Duplex results and arteriographic results for the internal carotid artery, using the shon focus scanhead.

Short Focus Scanhead

Duplex

\begin{tabular}{|c|c|c|c|c|c|c|}
\hline \multicolumn{7}{|l|}{ Arteriography } \\
\hline & $0 \%$ & $5-15 \%$ & $16-49 \%$ & $50-99 \%$ & $100 \%$ & \\
\hline $0 \%$ & 4 & 4 & & & & 8 \\
\hline $5-15 \%$ & 1 & 9 & 3 & & & $\mathbb{1 3}$ \\
\hline $16-49 \%$ & & 4 & 16 & 3 & & 23 \\
\hline $50-99 \%$ & & 1 & 2 & 25 & & 28 \\
\hline \multirow[t]{2}{*}{$100 \%$} & & & & & 5 & 5 \\
\hline & 5 & 18 & 21 & 28 & 5 & 77 \\
\hline
\end{tabular}

Out of the eight arteriographic normal internal carotid arteries, four were classified as normal by the Duplex method, hence four were overcalled. In the 5$15 \%$ diameter group the agreement between arteriographic and Duplex data was $69 \%$ (9/13). one side was undercalled and three sides were overealled. In the $16-$ $49 \%$ diameter reduction group the agreement was $70 \%$ (16/23), four sides were 
undercalled and three sides were overcalled by the Duplex method. In the $50-99 \%$ diameter reduction group the agreement was $89 \%$ (25/28), three sides were undercalled. In the total occlusion category (100\% diameter reduction) the agrement was $100 \%(5 / 5)$ between the arteriographic and Duplex data.

Externall Carotid Artery. Hardcopy output of a normal external carotid artery is shown in figure 26 . Note the flow to zero in late systole reflecting the relatively high resistance peripheral vascular bed supplied by this artery.

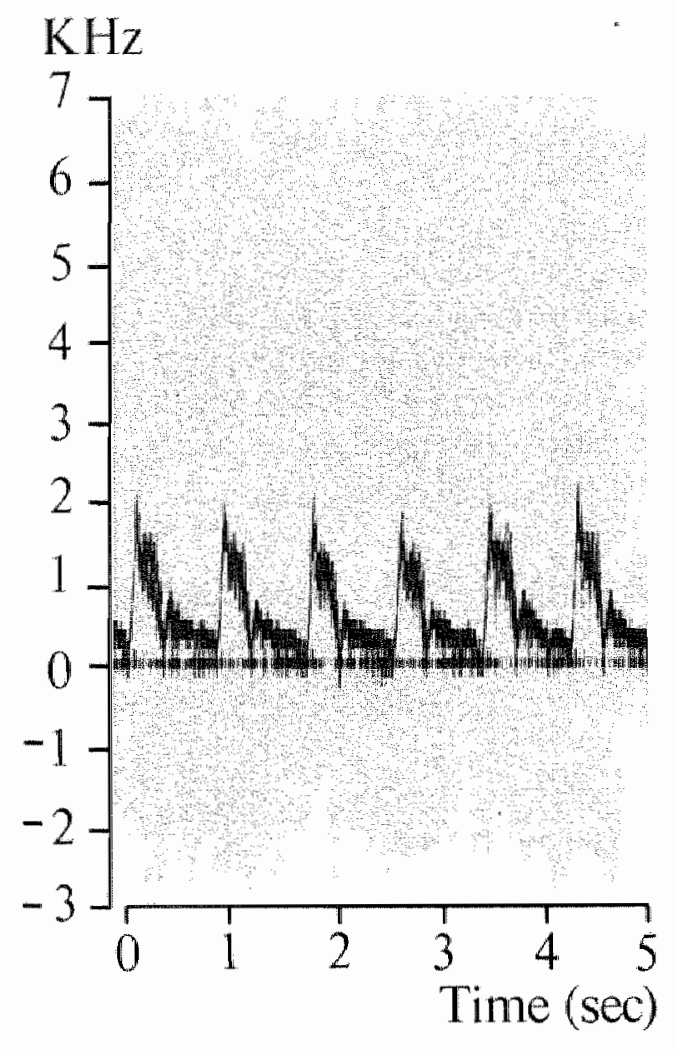

Figure 20.

Hardeopy of a normal cxemal warotid arlery signal. Note the thow to zero during dastole.

The results of the ultrasonic Duplex study compared to the results of multiplanar contrast arteriography for the external carotid artery using the medium focus scan head are shown in table VIII. Regarding the contrast arteriographic data as "gold standard", $48 \%(81 / 68)$ of the external carotid arteries were classilied as normal (0\% diameter reduction). $38 \%$ (64/168) were classified having a $5-49 \%$ diameter reduction, $12 \%(19 / 168)$ were classified having a $50-99 \%$ diameter reduction and $2 \%(4 / 168)$ were classified having a total occlusion (100\% diameter reduction).

Out of the 81 arteriographic normal external carotid arteries, agreement with the Duplex data was found in $25 \%$ (20/81). In the $5-49 \%$ diameter reduction category the agreement with the Duplex data was found in $67 \%$ (42/64). In the 50-99\% diameter reduction category the agreement was $84 \%(16 / 19)$, and in the total occlusion category (100\% diameter reduction) the agreement was $50 \%(2 / 4)$. 
Table Will.

Correlation of Duplex resuls and arteriographic results for the extemal carotid antery, using the medium focus scanhead.

Medium Focus Scanhead

Duplex

\begin{tabular}{c|c|c|c|c|c} 
Arteriography & $0 \%$ & $5-49 \%$ & $50-99 \%$ & $100 \%$ & \\
\hline $0 \%$ & 20 & 57 & 4 & & 81 \\
\hline $5-49 \%$ & 9 & 42 & 13 & & 64 \\
\hline $50-99 \%$ & & 3 & 16 & & 19 \\
\hline $100 \%$ & & 1 & 1 & 2 & 24 \\
\hline & 29 & 103 & 34 & 2 & 168
\end{tabular}

The results of the ultrasonic Duplex Scanner compared to the results of multiplanar contrast arteriography for the external carotid artery using the short focus scan head are shown in table IX. Regarding the contrast arteriographic data as "gold standard", $47 \%(36 / 76)$ of the external carotid arteries were classified as normal ( $0 \%$ diameter reduction), $42 \%$ (32/76) were classified having a $5-49 \%$ diameter reduction, $8 \%(6 / 76)$ were classified have a $50-99 \%$ diameter reduction, and $3 \%(2 / 76)$ were classified having a total occlusion (100\% diameler reduction).

Table IX.

Correlation of Duplex resules and arteriographic results for the external catotid ancery, using the short focus scanhead.

Short Focus Scanhead

Duplex

\begin{tabular}{c|c|c|c|c|c} 
Arteriography & $0 \%$ & $5-49 \%$ & $50-99 \%$ & $100 \%$ & \\
\hline $0 \%$ & 6 & 29 & 1 & & $\mathbf{3 6}$ \\
\hline $5-49 \%$ & 3 & 21 & 8 & & $\mathbf{3 2}$ \\
\hline $50-99 \%$ & & 1 & 4 & 1 & $\mathbf{6}$ \\
\hline $100 \%$ & & & 1 & $\mathbf{1}$ & 2 \\
\hline & $\mathbf{9}$ & $\mathbf{5 1}$ & $\mathbf{1 4}$ & $\mathbf{2}$ & $\mathbf{7 6}$
\end{tabular}


Out of the 36 arteriographic normal axternal carotid arteries, agreement with the Duplex data was found in $17 \%(6 / 36)$. In the $5-49 \%$ diameter reduction category the agreement between the arteriographic and Duplex data was $66 \%$ (21/32). In the $50-99 \%$ diameter reduction category the agreement was $67 \%$ (4/6), in the total occlusion category (100\% diameter reduction) one out of two was classified correctly.

\subsubsection{Discussion.}

Noninvasive methods such as pulsed Doppler and spectral analysis allow the use of parameters such as peak frequency and spectral width to categorize carotid artery disease. It is essential, however, to realize the potential and limitation of the methods in order to properly interpret the acquired data. For instances information about the angle between the Doppler beam and axis of the vessell is important, since it affects the recorded frequency shift which is used in classifying the degree of stenosis (see 5.2 .1 .). Given the availability of the B-mode image, it is used to measure the angle of the sound beam to the long axis of the vessel. With the present scan head, it is relatively easy to utilize an angle close to 60 degrees cven in the distal internal carotid artery.

Since spectral width is used as one of the parameters to classify severity of disease, it is essential to place the sample volume in the centerstream of the vessel.

Approaching the wall there will be velocity gradients even in normal vessels. The increase in the range of frequencies, produced by these gradients, will be recorded as spectral broadening. The short locus scan head provides a sample volume at its focus range which is a factor of 8 smaller than with the medium focus transducer (see 4.3.2.). The smaller sample volume has a theoretical advantage in these patients where spectral broadening is used to differentiate between normal arteries and those with minimall disease.

Internal carotid artery. There were 169 internal carotid arteries evaluated using the medium locus scan head. Twelve of these were called normal by contrast anteriography. Of these welve normal sides only one was classified normal by Duplex scanning. One of the explanations for this poor agreement could be the lact that arteriography is nol an ideal "gold standand" for classifying normal carotid arterics.

Because the arleriograms in this study were reported independenlly by two radiologists, we had the opportunily of evaluating the agreement between two radtiologists in reporting varying degrees of stenoses as defined by the categories previously mentioned for the internal carotid artery ${ }^{41}$. The likelihood of two radiologists agreeing that a carotid biturcation is normal, was only $56.8 \%$. When the sume observer reviewed the films on two separate occasions, agreement occurred $74.8 \%$ of the time. This rases the question of what is the optimum method for identifying normal carotid arteries as the standard for comparison against noninvasive methods.

There are several possible methods available for defining the extent of stenosis from the arteriogram. In this study, the method used the unsubstracted arteriogram becaluse the presence of calcification in the outer layers of the vessel 
wall in the region of the stenosis greatly facilitates the estimation of the normal vessel diameter. We have expressed the degree of stenosis as the percent diameter reduction of the normal wall diameter at the level of the stenosis. Othet authors have measured the minimal transvere diameter and have compared this to the diameter of the internal carotid artery beyond the region involved in the disease process ${ }^{57}, 12 \%$. This measurement technique is mainly used by instifutions where indirect noninwasive studies are performed to estimate the extent of extracraniat carotid artery disease. For a direct noninvasive test this measurement technique maty well miss or underestimate lesions of the carotid bub. Obviously the difficulties involved in the interpretation of carotid arteriograms 41 . 47 . st present a serious problem in the evaluation of the accuracy of noninvasive methods.

The second feature of note is the fact that there is a relatively small number of nomal internal carotid arteries available for evaluation. It should be noted that with few exceptions, the decision to perform arteriography was not the responsibility of the personnel performing the noninvasive study. The results of the Duplex scan were simply reported to the referring physician without any recommendation as to the subsequent management of the patient. This issue will be addressed in chapter 6.

With the medium focus scan head only one of the welve arteriographically normal carotid sides was called normal by the Duplex method. Identification of spectrat broadening (six out of the twelve normal internal carotid arteries were classified as a $5-15 \%$ diameter reduction) in normals might be caused by wariations in the position and size of the sample volume. With the smaller sample volume of the short focus scan head the agreement between arteriography and Duplex method for normal carotid arteries was $50 \%(4 / 8)$, which is an indication that with a smaller sample volume nomals may be identilied more accurately.

The disagreement in the four arteriographically normal internal arteries which were classified as $16-49 \%$ diameter reduction, using the medium focus scanhead, was of some concern. With the short focus scan head no nomals were classified as having a 16-49\% diameter reduction. This again suggests that a smallor sample volume provides a "cleaner" signal (less spectral broadening) from normal carotid arteries.

The most serious disagrement in the group of 12 arteriographically nomal internal carotid arteries, using the medium focus scanhead, was found in a pationt where a $50-99 \%$ diameter reduction was predicted. The arteriograms of this patient demonstrated a kink of the internal carotid artery, which was not recognized during the study (figure 27). The hardcopy of the spectrum of the internal carotid artery demonstrated systolic peak frequencies above $4 \mathrm{KH}$ with spectral broadening during systole. The high peak frequencies were caused by the steep (not noticed) angle between the Doppler beam and the axis of the vessel. The spectral broadening was presumably caused by an incorrect position of the sample volume. Since this arror occurred more attention has been devoled to the identification of kinked arteries.

The agreement between arteriographic and Duplex data in the $5-15 \%$ diatroter reduction category using the medium focus scanhead was $51 \%(19 / 37), 17$ sides 
were owercalled by the Duplex data. Of these 17 sides, 15 were classified as $16-$ $49 \%$ diameter reduction. The sample volume size of medium focus scanhead can be a reasonable explanation for the increased spectral broadening in these cases. More serious are the two sides which were classified as 50-99\% diameter reduction. An explanation for these two errors was not found when both the spectra and the arteriograms were reviewed.

The agreement between arteriographic and Duplex data in the 5-15\% diameter reduction category using the short focus scanhead was $69 \%(9 / 13)$, which is better than the agreement using the medium focus scan head $(51 \%)$. Only three sides were overalled, all were found in the 16-49\% diameter reduction category.

Combining the normals with the $5-15 \%$ diameter reduction category the agrement between atteriographic and Duplex data is $55 \%(27 / 49)$ for the medium focus and $86 \%(1821)$ for the short focus scanhead. This demonstrates that the short focus scanhead, providing a smaller volume, appears to be better for defining those palients with normal or minimally diseased carotid arteries (calegory $A$ and $B$ ).

In the $16-49 \%$ diameter reduction category the agreement between arteriographic and Duplex data using the medium locus scanhead was $59 \%$ (24/41). For the short focus scanhead this agreement was $70 \%(16 / 23)$, again higher than for the medium focus scanhead. Using the short focus scanhead three carotid sides were overcalled by the Duplex method. A review of the spectra demonstrated systolic peak frequencies above $4 \mathrm{KHz}$ in each instance. The results in all groups with a diameter reduction less than $50 \%$ (category $\mathrm{A}, \mathrm{B}$ and $\mathrm{C}$ ) were better using the short focus scanhead, making this scanhead more applicable for detecting and differentiating normal and minimally diseased internal carotid arteries.

In the $50-99 \%$ diameter reduction category, the agreement between the two methods was $94 \%(61 / 65)$ using the medium focus scanhead. In one case an arteriographic $50-99 \%$ diameter reduction was called a total occlusion. Compared with early results of the Duplex method (table I) the accuracy in predicting a 50$99 \%$ diameter reduction has improved, which in part is a reflection of the appreciation of the characteristics of the common carotid artery (see 5.2.). Using the short focus scanhead the agreement between the arteriographic and Duplex data was $89 \%$, in no instance overcalled the Duplex method the arteriographic diameter reduction. This is reassuring since differentiating between a high-grade stenosis and a total occlusion is of great clinical importance.

In the total occlusion category the agreement between the two methods was $93 \%$ (13/14) using the medium focus scanhead and $100 \%(5 / 5)$ using the short focus scanhead. Only on one occasion was a arteriographic proven total occlusion undercalled by the Duplex method. The overall agreement in predicting a total occlusion combining the data from the medium and short focus scanhead was $95 \%$ (18/19). demonstrating that the Duplex concept is able to identify a total occlusion accurately. The fact that Duplex scanning in combination with spectral analysis is able to differentiate accurately between high grade lesions and total occlusions of the internal carotid artery makes this method superior to all indirect noninvasive methods currently in use. 
External carotid artery. The distribution of disease in the extemal carotid artery is different from that found in the internal carotid artery. Forty eight percent of the external carotid arteries (117/224) were normal by arteriography, this was only $8 \%$ (20/246) for the internal carotid arteries. An arteriographic $50-99 \%$ diameter reduction was lound in $10 \%(25 / 224)$ of the external carotid arteries and in $38 \%$ (93/246) of the internall carotid arteries.

The clinical information clesired for the extemal carotid artery is to differentiate between low-grade and high-grade lesions. For the latter it is important to differentiate between high grade stenoses and total occlusions. Combining the normal and $5-49 \%$ diameter reduction category the agreement between the arteriographic and Duplex data using the medium focus scanhead was $88 \%$ (128/ 145). Using the short focus scanhead the agreement was $86 \%$ (59/68), demonstrating that there is no difference using the two different scanheads. For the $50-99 \%$ reduction category the agreement between the two diagnostic methods using the medium focus scanhead was $84 \%$ (16/19). Using the shorf focus scanhead the agreement was $67 \%$ (4/6), combining the medium and short focus scanthead data the agreement was $80 \%(20 / 25)$. These percentages demonstrate that the Duplex method does differentiate between non-hemodynamic and hemodynamic diameter reduction reasonably well.

For the occlusion category the numbers are small, the overall agreement between the arteriographic and Duplex data combining the medium and short focus scanheads studies was $50 \%(3 / 6)$.

The reason that there is no marked difference between the medium and short focus scanhead is in the fact that the sample volume size of both systems is relatively large compared with the diameter of the external carotid artery.

\section{Difficult studies.}

Most problems are found in those cases where the anatomy of the extracranial carotid system is difficult to delineate. Kinking of the internal carotid artery, when not identified, creates a situation in which the technician can be misled due to a steep angle between the Doppler beam and the axis of the vessel (figure 27).

Another problem can arise when the first branch of the external carotid artery (superior thyroid artery) is incorrectly identified as the proximal internal carotid artery. The Doppler spectrum from this small artery will show high systolic peak frequencies with marked spectral broadening. This problem can be anticipated by consequently identifying the proximal internal carotid artery just distal to the carotid bulb. 


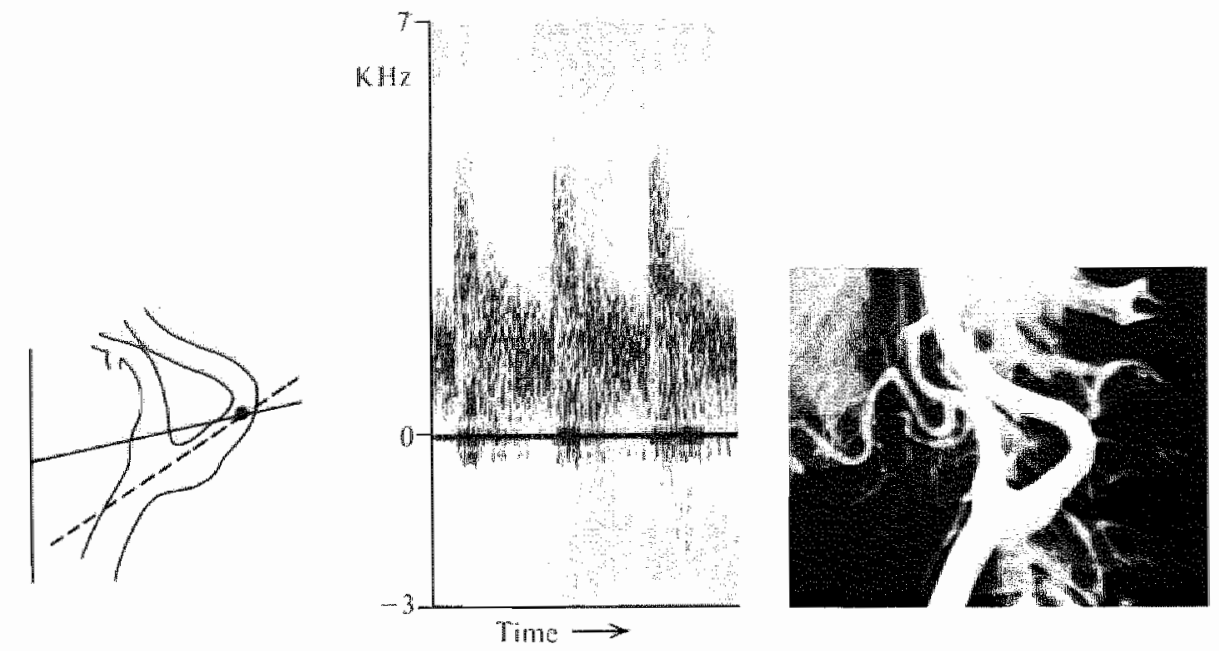

Hegne 27

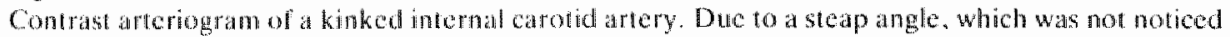
during the norinvasive study, the velocity pallern was classified as 50-99o diametrer reduction.

There are situations in which the external carotid artery velocity patterns are difficult to differentiate from the internal carotid artery velocity signals. This can occur with a total occlusion of the internal carotid artery where the external carolid artery functions as a collateral for the ipsilateral hemisphere. Due to the

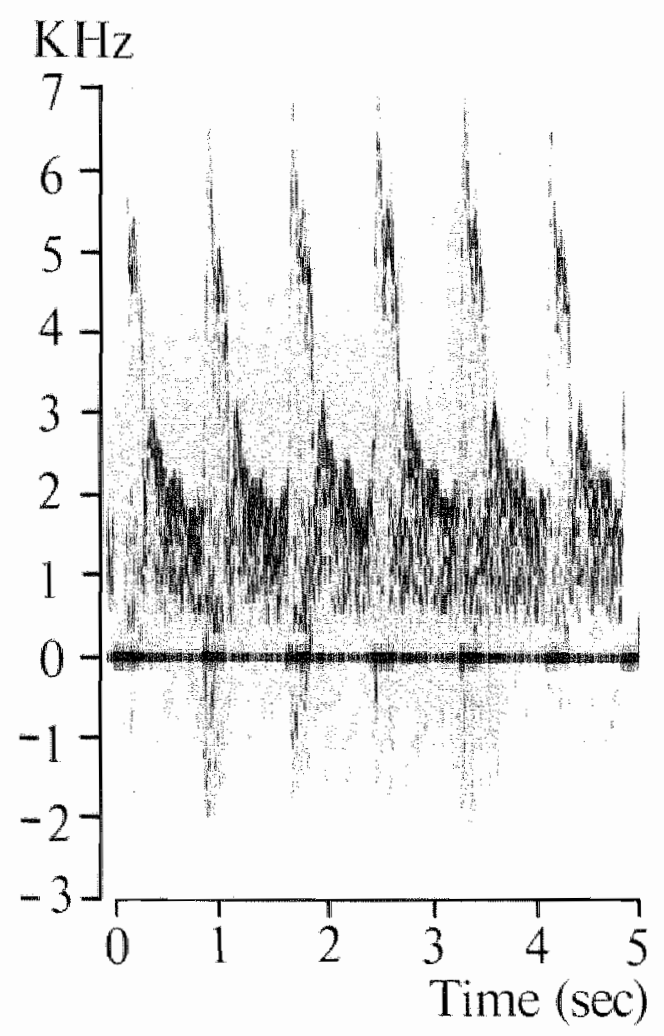

Figure 28.

Hardcopy of an external carotid artery signal in a patient with a tala ocelusion of the internat carotidartery. Note the high frequencies during diastole. 
low resistance of the intracerebral vascular bed, flow remains well above zero during diastole in the external carotid artery (see figure 28 ). One should be aware of this situation when velocity signals from only one artery can be identified above the carotid bifurcation.

A typical velocity signal can be obtained when the sample volume of the Doppler system is placed within the stenosis. Turbulence normally occurs just distal to the stenosis. In the stenosis itself high systolic frequencies will be found due to a velocity increase. The signal from the stenosis itself will be without spectral broadening (see figure 29). Whenever this type of signal is noted, the sample volume should be moved more distally to obtain the expected marked spectral broadening which is typical for a high grade stenosis.

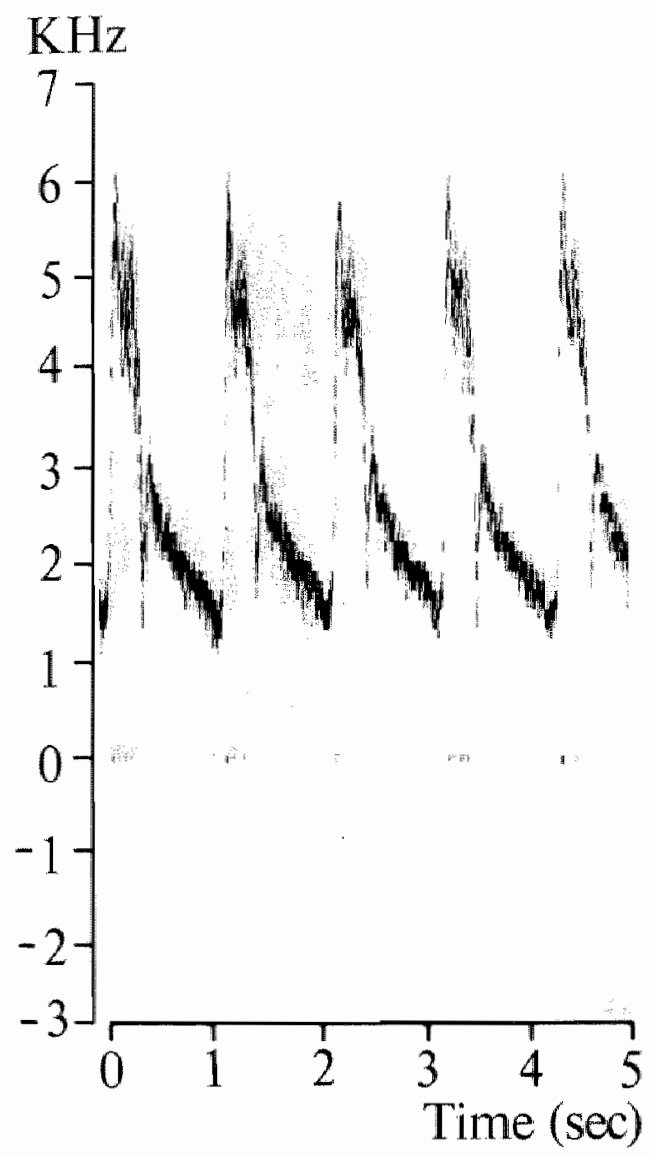

Figure 29

Hardoppy of an internal carodid artery signal. The sample volume is localized within a highgrade stconosis. Note the high systolic and diastolic frequencics and the absence of spectral broadening.

A difficult situation can occur when a patient with an occlusion of the common carotid artery is studied. The occlusion is diagnosed whenever no velocity signal can be obtained in the region of the common carotid artery. One should be aware of the fact, that in this situation the homolateral external carotid artery can by reversal of flow, supply blood to the internal carotid artery ${ }^{2 \%}$. Figures $30-32$ show how important the directional flow information is in such a study. Figure 30 shows the Doppler spectrum from the bulb, with velocities both in the forward and 
reverse direction. Blood flow to the external carotid artery is due to collateral filling in the reversed direction (figure 31 ), with flow in the internal carotid artery in the forward direction (figure 32). This study shows that common carotid artery occlusion is not invariably associated with trombosis of the internal carotid artery. Furthermore, the study demonstrates that whenever an occlusion of the common carotid artery is found, velocity signals in the internal and external carotid artery can be detected.

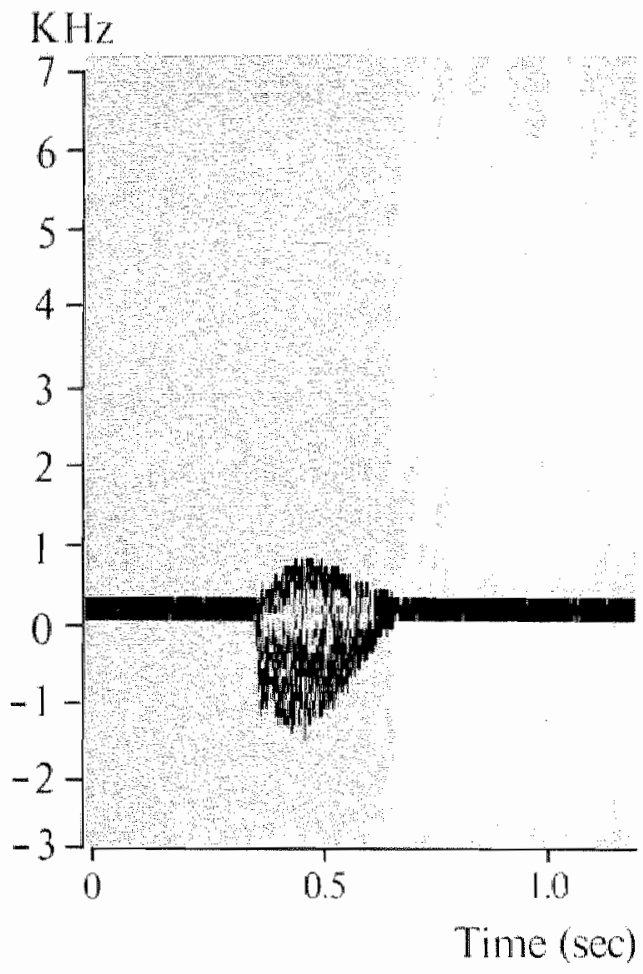

Figure 30 .

Hardcopy of a signat from the carotid bulb in a paticn with an occlusion of the common carotid artery "There ane frequencies present both in the foward and reverse direction. 


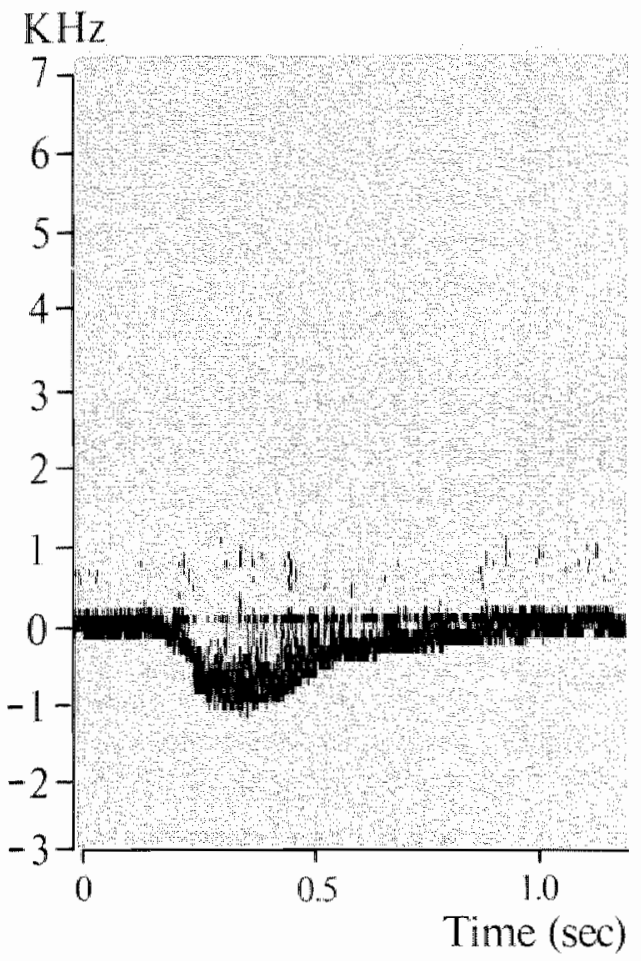

$\mathrm{KHz}$

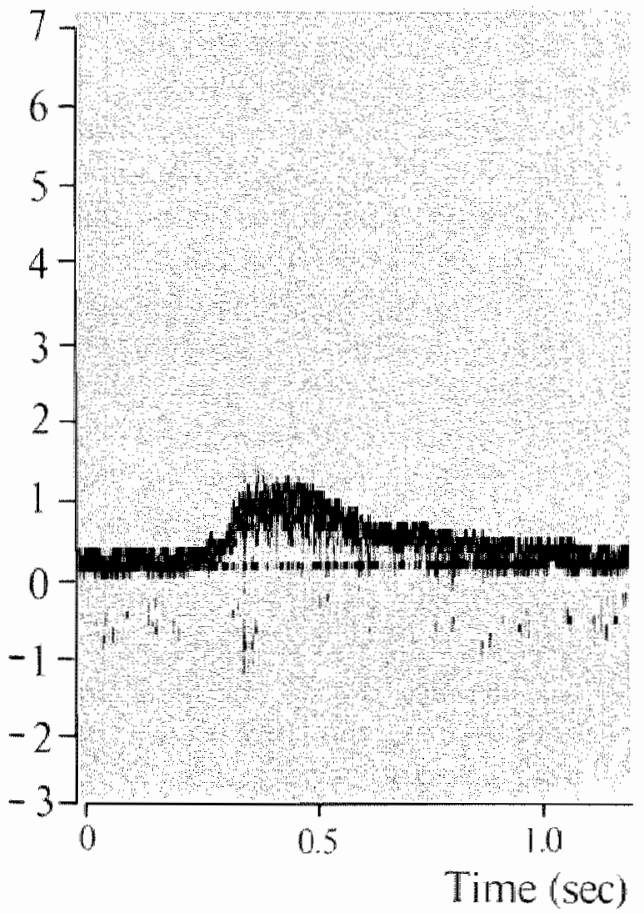

Figute 31.

Harcopy of a signal from the external carotid artery of the same pationt. The flow in this artery is in the reverse direction the to collaterai blood supply.
Tigure 32.

Hardcopy of a signal from the internal canotid artery of the same paticnt. The flow in this artery is if the florward direction. 


\section{Conclusions.}

The presented data has demonstrated that in the hands of experienced technicians, following a standard protocol, Duplex scanning combined with spectral analysis is an accurate method in predicting noninvasively the extent of internal carotid artery disease. For identifying normal and minimal diseased internal carotid anteries (5-15\% diameter reduction and 16-49\% diameter reduction callegory) the short focus scanhead improves the accuracy of the method significantly. For identifying hemodynamically significant diameter reductions $(50-99 \%$ diameter reduction and $100 \%$ diameter veduction category) of the internal carotid artery, there is no significant difference in the accuracy using the medium or short focus scanhead. The capability to differentiate between a high grade stenosis and a total occlusion makes the method superior to any indirect noninvasive test.

The accuracy of the Duplex concept justifies the use of this method as a screening device for patients with suspected carotid artery disease. Furthermore, the method has the potential to document progression of arterial disease when it is used in follow-upstudies.

\subsection{Effect of carbon dioxide on flow patterns in normal extracranial arteries.}

\subsubsection{Introduction.}

The results of the Duplex scanner studies in identifying normal carotid arteries and differentiating them from minimal diseased carotid arteries should still improve. The problems we encounter concerning this issue are complex. In the first place the number of normal arteriogrammed carotid arteries remains small as a result of the influence of our test on the management of referring physicians (see 6.2.3.). Secondly, we have to realize that arteriography, especially in identifying normals, is not an ideal gold standard "II.

'The change in equipment, i.e. the short focus scan head improved the accuracy of normal and minimal diseased internal carotid arteries considerably (see 5.3.). With this short focus scan head still $50 \%$ of the normal internal carotid arteries were classified as a $5-15 \%$ diameter reduction. Since it is well known that tesser degrees of stenoses may be "unmasked" by increasing flow rates across the lesion, we considered to increase the blood flow to the brain in patients with minimal carotid disease. However, before applying this method to patients it was necessary to evaluate the velocity pattern changes due to an increase in total blood flow of normal subjects. As a pilot study we gave five normal subjects carbon dioxide. For the purpose of the present study velocity pattern recordings were made from the centerstream of three sites in each carotid system using the Duplex scanner: (1) low common carotid artery; (2) proximal internal carotid artery; and (3) proximal external carotid artery. Since welocity changes in diastole are closely related to changes in total blood flow ${ }^{144}$, the following hand measured parameters were used to document changes in flow in the carotid arteries: 
(1) velocity $(\mathrm{cm} / \mathrm{sec})$ in diastole of the common carotid artery $(500$ mseconds after peak systole)

(2) velocity $(\mathrm{cm} / \mathrm{sec})$ in diastole of the internal carotid artery $(500$ mseconds after peak systole)

(3) velocity $(\mathrm{cm} / \mathrm{sec})$ in diastole of the external carotid artery $(500$ mseconds after peak systole)

The velocity signal, the incident angle of the sound beam and ECG signal were simultaneously recorded on a four channel audiotape recorder. "The output of the audiotape was first analyzed with the fast Fourier Transform spectrum analyzer configured to output 100 bins of frequency information every $2.5 \mathrm{msecond}$ in digital format which together with the ECG timing signal was read to disk. The information recorded on disk was then subjected to a computer program designed to measure specified parameters of the velocity waveform ${ }^{73}$. The spectra of twenty heart beats were averaged to form an ensemble waveform. The frequency with the highest amplitude (termed "the mode") of this averaged spectrum was computed. Contour lines calculated $9 \mathrm{~dB}$ above and below the mode were plotted thus allowing measurements of the spectral width to be made at any desired point of the pulse cycle. The following waveform parameters were used for the analysis:

(1) velocity in peak systole at upper $9 \mathrm{~dB}$ line in the common carotid artery

(2) velocity in peak systole at upper $9 \mathrm{~dB}$ line in the internal carotid artery

(3) spectral width between $9 \mathrm{~dB}$ lines in the deceleration phase of systole in the common carotid artery.

(4) spectral width between $9 \mathrm{~dB}$ lines in the deceleration phase of systole in the internal carotid artery.

Five healthy volunteers were used for this study. After 15 minutes of rest in the supine position, baseline heart rate and blood pressure were recorded. The carotid arteries were studied with the Duplex scanner and the subject breathing room air. The volunteer then started to breathe a mixture of $6.8 \%$ carbon dioxide and $93.2 \%$ oxygen. This mixture was chosen as it has been shown to increase the total cerebral blood flow by approximately $75 \%{ }^{99,152}$. After two minutes and while continuing to breathe the gas mixture, heart rate and blood pressure were again recorded. The two minute interval was chosen, as it has been shown that the response to carbon dioxide is maximal after twenty seconds and after two minutes remains stable ${ }^{99}$. Ten sides in five subjects were available for evaluation both prior to and while breathing carbon dioxide.

\subsubsection{Results.}

There were no significant changes found in the pulse rate or blood pressure during breathing 6.8 percent carbon dioxide. Examples of the hardcopy of the spectral analysis prior to and during breathing carbon dioxide are shown in figures 33 to 

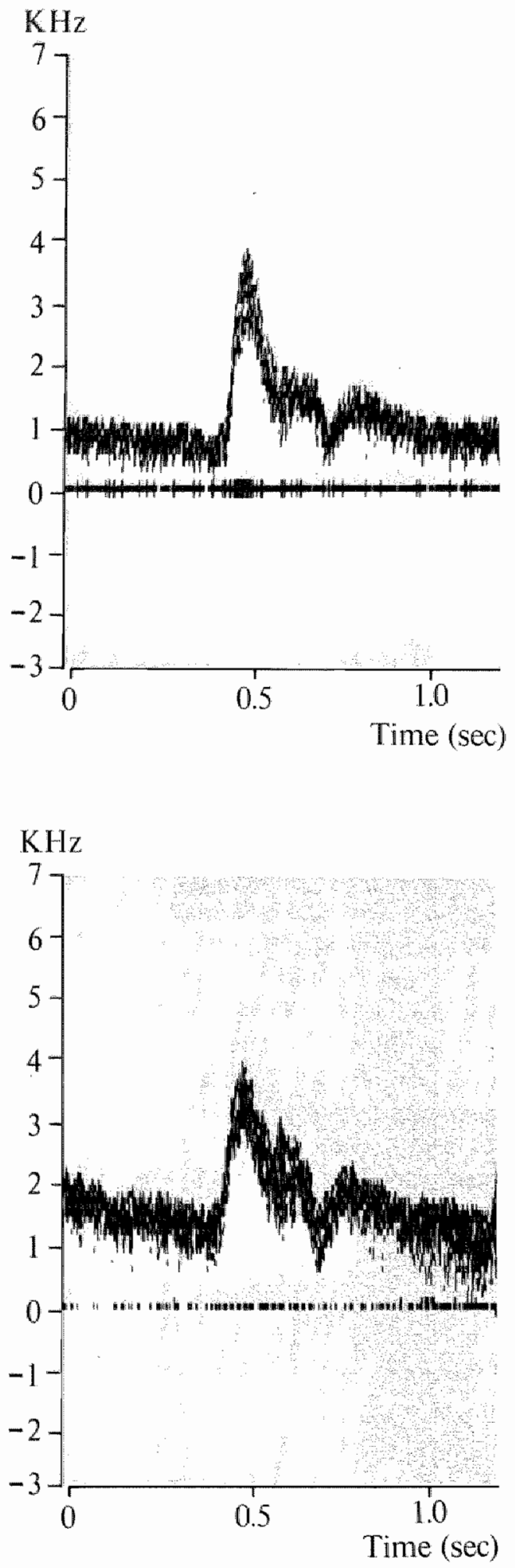

Figure 33.

Pulsed Doppler spectrum from a normal common carotid artery prior to breathing carbon dioxide.
Figure 34.

Pulsed Doppler spectrum from the same common carotid artery during breathing arbon dioxide. 

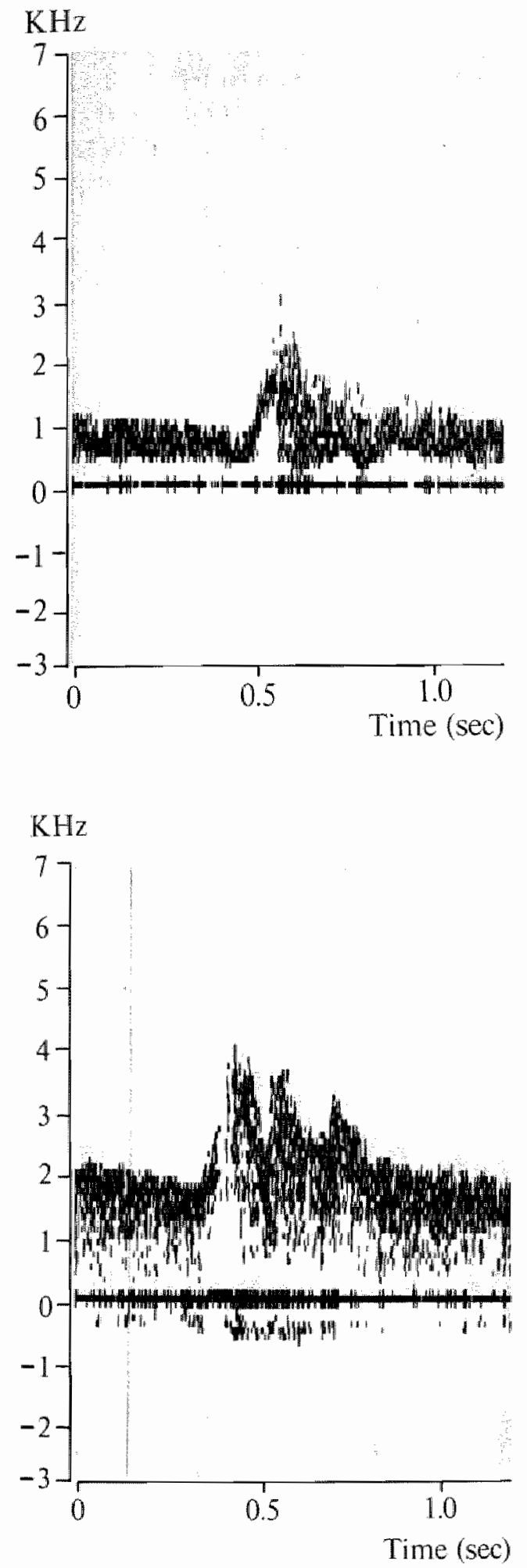

Figure 35

Pulsed Doppler spectrum from a normal internal carotid artery prior to breathing carbon dioxide.
Figure 36.

Pulsed Doppler spectrum from the same internal caroniditery during breathing carbon dioxide. 
36. There was an increase in diastolic frequencies both in the common carotid artery (figure 34) and the internal carotid artery (figure 36) under the influence of breathing carbon dioxide. The velocity in diastole ( $500 \mathrm{mseconds}$ after peak systole) prior to and during breathing carbon dioxide for the common, internal and external carotid artery are shown in table $\mathrm{X}$. The mean velocity in diastole of both sides of the five subjects increased by $106 \%$ in the common carotid artery. The difference from side to side in one subject and the difference between subjects however should be noted. The mean velocity in diastole of both sides in the internal carotid artery of the five subjects increased by $96 \%$. Again a marked spread was noted between the sides within one subject and between the subjects. The mean velocity in diastole of both sides of the five subjects increased by $21 \%$ in the external carotid artery. On one side we were unable to obtain a satisfactory hardcopy of the velocity pattern of the external carotid artery.

Table X.

Velocity (cm/sec) prior to and during breathing carbon dioxide in diastole ( $500 \mathrm{msec}$ after peak systole) from the common, internal and external carotid artery.

\begin{tabular}{|c|c|c|c|c|c|c|c|c|c|}
\hline & \multicolumn{3}{|c|}{ Common Carotid Artery } & \multicolumn{3}{|c|}{ Internal Carotid Artery } & \multicolumn{3}{|c|}{ External Carotid Artery } \\
\hline & $\begin{array}{c}\text { Prior } \\
(\mathrm{cm} / \mathrm{sec})\end{array}$ & $\begin{array}{c}\text { During } \\
(\mathrm{cm} / \mathrm{sec})\end{array}$ & \% Change & $\begin{array}{c}\text { Prior } \\
\text { (crm/sec) }\end{array}$ & $\begin{array}{c}\text { During } \\
(\mathrm{cm} / \mathrm{sec})\end{array}$ & $\%$ Change & $\begin{array}{c}\text { Prior } \\
(\mathrm{cm} / \mathrm{sec})\end{array}$ & $\begin{array}{c}\text { During } \\
(\mathrm{cm} / \mathrm{sec})\end{array}$ & \% Change \\
\hline No. I: $\mathbb{R}$ & 15 & 33 & 120 & 30 & 57 & 90 & 24 & 35 & 45 \\
\hline L & 25 & 46 & 84 & 23 & 46 & 100 & 24 & 24 & $-0-$ \\
\hline No. II: $\mathrm{R}$ & 24 & 49 & 104 & 30 & 46 & 53 & 16 & 17 & 6 \\
\hline & 32 & 50 & 56 & 21 & 42 & 100 & 27 & 38 & 40 \\
\hline No III: $\mathrm{R}$ & 17 & 39 & 120 & 17 & 41 & 141 & 16 & 23 & 43 \\
\hline $\mathbb{L}$ & 17 & 43 & 153 & 27 & 49 & 81 & 8 & 7 & -12 \\
\hline No. IV: R & 24 & 55 & 129 & 21 & 48 & 128 & 18 & - & - \\
\hline $\mathbb{L}$ & 36 & 63 & 75 & 29 & 61 & 110 & 20 & 22 & 10 \\
\hline No. $\mathrm{V}: \mathrm{R}$ & 15 & 38 & 153 & 23 & 41 & 78 & 20 & 20 & -0 \\
\hline $\mathrm{L}$ & 23 & 37 & 60 & 23 & 42 & 82 & 17 & 27 & 58 \\
\hline $\begin{array}{l}\text { Mean } \\
( \pm S D)\end{array}$ & $\begin{array}{c}22 \\
( \pm 7)\end{array}$ & $\begin{array}{c}45 \\
( \pm 9)\end{array}$ & $106 \%$ & $\begin{array}{c}24 \\
( \pm 4)\end{array}$ & $\begin{array}{l}47 \\
( \pm 6)\end{array}$ & $96 \%$ & $\begin{array}{c}19 \\
( \pm 5)\end{array}$ & $\begin{array}{c}23 \\
( \pm 9)\end{array}$ & $21 \%$ \\
\hline
\end{tabular}


Examples of the computer hardcopy of the averaged spectrum prior to and during breathing carbon dioxide are shown in figure 37 to 40 . Under the influence of carbon dioxide the frequencies in diastole in the common carotid artery (figure 38)

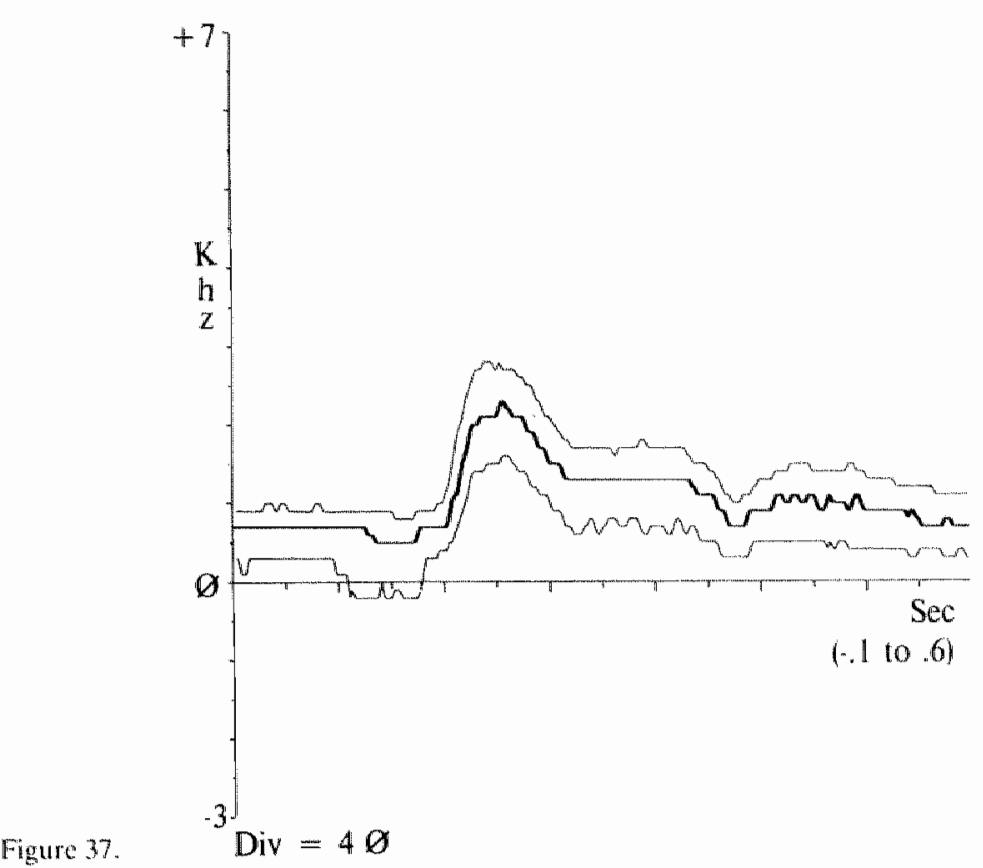

Computer hardcopy output of an averaged spectrum from a nomal common carotid artery prior to breathing arbon dioxide.

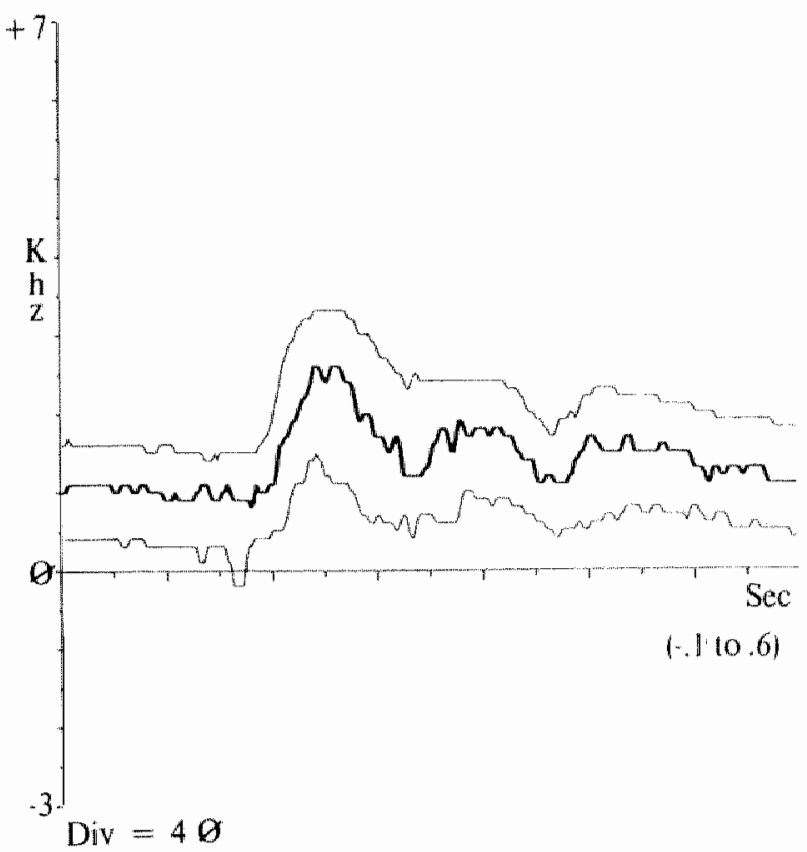

Fighre 38 .

Div $=4 \varnothing$

Computer hardcopy output of the same common carotid artery during breathing carbon dioxide. 


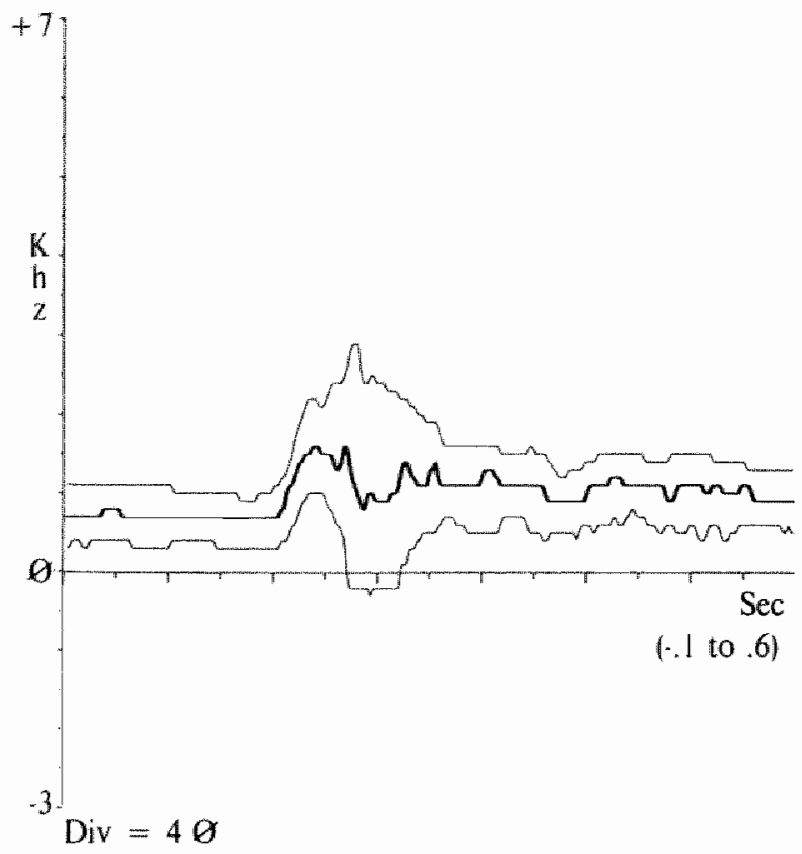

Figure 39. $\quad$ Div $=4 \theta$

Computer hardcopy output of an averaged spectrum from a normal internal carotid artery prior to breathing carbon dioxide.

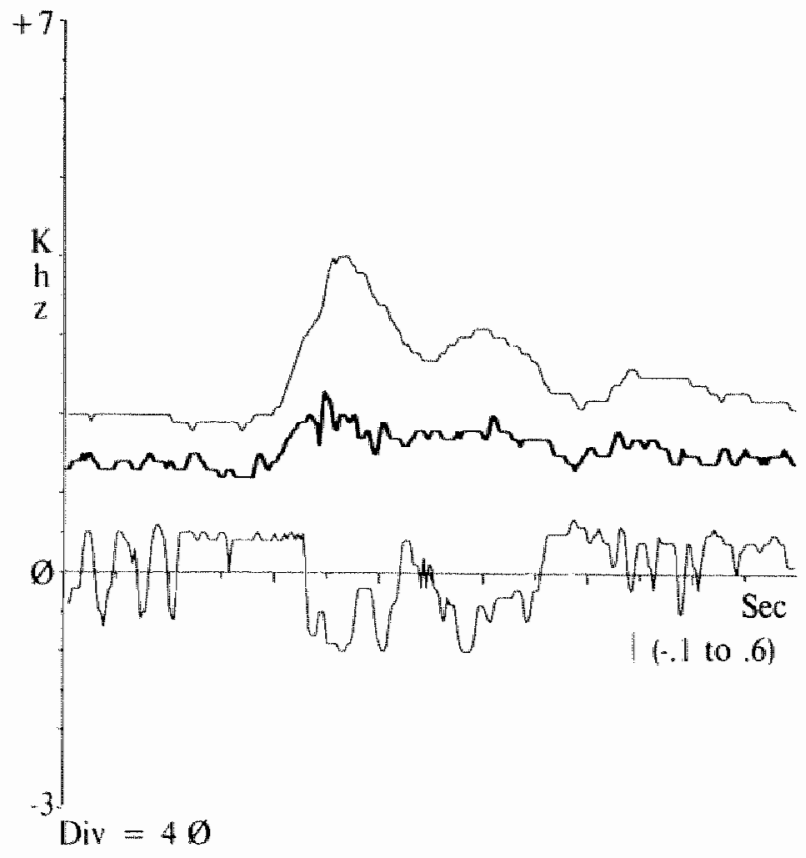

Figure 4 io.

Computer harteopy ontput of the same intermal carotid artery during breathing carbon dioxide. 
and the internal carotid artery (figure 40) are markedly increased. The velocity at peak systole measured at the upper $9 \mathrm{~dB}$ line of the common carotid artery and internal carotid artery are shown in table XI. The mean common carotid peak

\section{Table XI.}

Velocity $(\mathrm{cm} / \mathrm{sec})$ prior to and during breathing carbon dioxide at peak systole from the comnon and internal carotid artery.

\begin{tabular}{|c|c|c|c|c|c|c|c|}
\hline \multicolumn{2}{|l|}{ Subject } & \multicolumn{3}{|c|}{ Common Carotid Artery } & \multicolumn{3}{|c|}{ Internal Carotid Artery } \\
\hline & & Prior & During & $\%$ Change & prior & During & change \\
\hline \multirow[t]{2}{*}{ No. I: } & $\mathrm{R}$ & 84 & 110 & 30 & 84 & 131 & 55 \\
\hline & $\mathrm{L}$ & 118 & 123 & 4 & 84 & 138 & 64 \\
\hline \multirow[t]{2}{*}{ No. II: } & $\mathrm{R}$ & 139 & 155 & 11 & 69 & 123 & 78 \\
\hline & $\mathrm{L}$ & 139 & 152 & 9 & 84 & 113 & 33 \\
\hline \multirow[t]{2}{*}{ No. III: } & $\mathrm{R}$ & 144 & 138 & -4 & 76 & 110 & 44 \\
\hline & L & 144 & 130 & -9 & 109 & 113 & 3 \\
\hline \multirow[t]{2}{*}{ No. IV: } & $\mathrm{R}$ & 164 & 166 & $\mathbb{1}$ & 91 & 110 & 20 \\
\hline & $\mathrm{L}$ & 191 & 210 & 10 & 82 & 130 & 59 \\
\hline \multirow[t]{2}{*}{ No. V: } & $\mathrm{R}$ & 69 & 69 & -0 & 53 & 90 & 67 \\
\hline & L. & 84 & 104 & 23 & 46 & 70 & 53 \\
\hline $\begin{array}{l}\text { Mean } \\
( \pm \text { SD })\end{array}$ & & $\begin{array}{c}127 \\
( \pm 38)\end{array}$ & $\begin{array}{c}135 \\
( \pm 38)\end{array}$ & $7 \%$ & $\begin{array}{r}77 \\
( \pm 18)\end{array}$ & $\begin{array}{c}112 \\
( \pm 20)\end{array}$ & $47 \%$ \\
\hline
\end{tabular}

systolic velocity of both side of the five subjects increased by $7 \%$ during breathing carbon dioxide. The mean internal carotid peak systolic velocity of both sides of the five subjects increased by $47 \%$ during breathing carbon dioxide. The spectral widths between the $9 \mathrm{~dB}$ lines in the deceleration phase of systole in the common carotid artery are shown in table XII. The mean spectral width of the both sides of the five subjects increased by $51 \%$. The spectral widths between the $9 \mathrm{~dB}$ lines in 
Table Xll.

Spectrat with (KHz) betwen the $9 \mathrm{~dB}$ lines in the deceleran phase of systole from the common corotidariery.

\begin{tabular}{|c|c|c|c|c|}
\hline \multicolumn{2}{|l|}{ Subject } & \multicolumn{3}{|c|}{ Common Carotid Artery } \\
\hline & & Prior & During & change \\
\hline \multirow[t]{2}{*}{ No. 1: } & $\mathrm{R}$ & 0.23 & 0.43 & 87 \\
\hline & $\mathbb{L}$ & 0.29 & 0.36 & 24 \\
\hline \multirow[t]{2}{*}{ No. II: } & $\mathrm{R}$ & 0.35 & 0.54 & 54 \\
\hline & $\mathbb{L}$ & 0.39 & 0.70 & 79 \\
\hline \multirow[t]{2}{*}{ No. III } & $\mathrm{R}$ & 0.56 & 0.79 & 41 \\
\hline & $\mathbb{L}$ & 0.55 & 0.71 & 29 \\
\hline \multirow[t]{2}{*}{ No. IV: } & $\mathrm{R}$ & 0.69 & 0.53 & -23 \\
\hline & $\mathbb{1}$ & 0.77 & 0.93 & 20 \\
\hline \multirow[t]{2}{*}{ No. V: } & $\mathrm{R}$ & 0.116 & 0.25 & 56 \\
\hline & $L$ & 0.17 & 0.42 & 147 \\
\hline $\begin{array}{l}\text { Mean } \\
( \pm S D)\end{array}$ & & $\begin{array}{c}0.41 \\
( \pm 0.21)\end{array}$ & $\begin{array}{c}0.56 \\
( \pm 0.21)\end{array}$ & $51 \%$ \\
\hline
\end{tabular}

Table XIII.

Spectrat width $(\mathrm{KHZ})$ between the $9 \mathrm{~dB}$ lines in the deceleration phase of systole from the internal carotid artery.

\begin{tabular}{|c|c|c|c|c|}
\hline \multicolumn{2}{|l|}{ Subject } & \multicolumn{3}{|c|}{ Internal Carotid Artery } \\
\hline & & Prior & Duringe & of Change \\
\hline \multirow[t]{2}{*}{ No. I: } & $\mathrm{R}$ & 0.55 & 0.94 & 70 \\
\hline & $\mathbb{L}$ & 0.56 & 0.67 & 20 \\
\hline \multirow[t]{2}{*}{ No. II: } & $\mathrm{R}$ & 0.29 & 0.51 & 75 \\
\hline & $\mathbb{L}$ & 0.46 & 1.06 & 130 \\
\hline \multirow[t]{2}{*}{ No. III: } & $\mathrm{R}$ & 0.68 & 0.82 & 20 \\
\hline & $\mathbb{L}$ & 0.85 & 0.81 & -4 \\
\hline \multirow[t]{2}{*}{ No. IV: } & $R$ & 0.36 & 0.73 & 102 \\
\hline & $\mathrm{L}$ & 0.69 & 1.07 & 55 \\
\hline \multirow[t]{2}{*}{ No. V: } & $\mathrm{R}$ & 0.13 & 0.32 & 146 \\
\hline & L & 0.18 & 0.35 & 94 \\
\hline $\begin{array}{l}\text { Mean } \\
( \pm \text { SD })\end{array}$ & & $\begin{array}{c}0.47 \\
( \pm 0.23)\end{array}$ & $\begin{array}{c}0.72 \\
( \pm 0.26)\end{array}$ & $70 \%$ \\
\hline
\end{tabular}


the deceleration phase of systole in the internal carotid artery are shown in table XIII. The mean spectral width of both sides of the five subjects increased by $70 \%$ breathing $6.8 \%$ carbon dioxide.

\subsection{Discussion.}

Doppler devices have been used to evaluate changes in blood flow of the extracranial carotid arteries induced by breathing varying concentrations of carbon dioxide ${ }^{121,144}$. Inhalation of carbon dioxide produces a marked increase in the otherwise constant cerebral blood flow $63,128,131,152$

Risberg and Smith ${ }^{144}$, in a comparative study between continuous wave Doppler and Xenon inhalation, demonstrated that velocity changes during diastole are reliable indices of the increase in total cerebral blood flow. In the present study the mean peak velocity in diastole ( 500 mseconds after peak systole) of the common carotid artery and internal carotid artery increased approximately $100 \%$, an increase which correlated well with the increase in total blood flow induced by breathing $6.8 \%$ carbon dioxide. However, the variations noted between individuals were not inconsiderable. These variations can be explained by errors in the measurements or by true physiological differences that occur between different normal subjects and between the two sides of the same subject.

The breathing of carbon dioxide should not have a significant effect on the peripheral resistance of the vascular bed supplied by the external carotid artery. The present findings confirmed this expectation, the mean velocity in diastole of the external carotid artery only increased by $21 \%$ during carbon dioxide inhalation. Again however, there was a marked variation between the five subjects.

A major change in the velocity patterns induced by breathing carbon dioxide was the increase in spectral width. In vessels in which laminar flow is present, the velocities of red blood cells within the sample volume of the pulsed Doppler device are relatively similar. Spectral analysis of the velocities within such a sample will show a narrow band of frequencies ${ }^{60}$. With disappearances of laminar flow the movement of red blood cells in the sample volume becomes more random, this will be reflected as a greater variation in frequencies, i.e. an increase in spectral width. In this study, when spectral width was measured in the deceleration phase of systole in the common and internal carotid artery, a $51 \%$ increase was observed in the common carotid artery after breathing carbon dioxide while in the internal carotid artery an increase of $70 \%$ was observed. The observed change in the spectral width is assumed to be related to the change in the flow pattern across the bifurcation that occurred secondary to the marked increase in flow.

Previous studies have used both qualitative and quantitative changes in spectral width to grade the degree of stenosis in the internal carotid artery. With disease at the carotid bulb, there is an initial increase in spectral width occuring first during the deceleration phase of systole, which comes to occupy the entire pulse cycle as the stenosis approaches a $50 \%$ reduction in diameter. For stenoses of less than $50 \%$ increases in peak systolic velocity are minimal with most emphasis placed upon the time of onset of spectral broadening. However, the spectral changes 
observed with early disease are often minimal and difficult to precisely classify. Thus, the separation of patients with minimal lesions from those who are considered arteriographically normal is a problem. The present study shows quite clearly that the increase in cerebral blood flow dramatically altered the velocity pattern in normal internal carotid arteries. While this type of provocative test is simple to perform, further studies will be required in patients with minimal extracranial arterial disease to determine if it is useful in increasing the sensitivity of the method in detecting minor degrees of stenoses.

\subsection{Algorithm for categorization of high grade stenoses.}

\subsubsection{Introduction.}

The accuracy of predicting a 50-99\% diameter reduction, using Duplex scanning combined with spectral analysis, is sufficient enough to justify the method as a screening device for patients with suspected extracranial carotid artery disease. However, for the purpose of documenting progression of disease, the $50-99 \%$ diameter reduction group is too broad. In order to estimate the degree of involvement within this category we evaluated an algorithm utilizing information from both the common and internal carotid artery.

In recent years, a great deal of effort has gone into the development of nominvasive algorithms to predict extracranial carotid artery disease. Planiol and Pourcelot ${ }^{134,135}$, using a continuous wave device, introduced the resistivity index (R.I.) measured from the velocity waveform of the common carotid artery to predict high grade stenoses and total occlusions of the internal carotid artery (see 2 2.3.). Rutherford et al. ${ }^{148}$, using a continuous wave device, introduced five hand measured parameters from the velocity waveform of the commmon carotid artery to predict extracranial carotid artery disease (see 2.2.3.). Archie ${ }^{1}$, using a similar Doppler device, introduced a "normalized" algorithm measured from the velocity wavelorm of the common carotid artery to predict severe internal carotid artery disease. All three methods were based on the hypothesis that disease in the internal carotid artery can be predicted by changes in the velocity waveform of the common carotid artery.

Blackshear et al. ${ }^{27}$ introduced the ratio of the mean peak internal carotid artery velocity to the mean peak common carotid artery velocity to predict severe internal carotid artery disease. This ratio was below 0.8 in all normals and above 1.5 in all 21 high-grade stenoses of the internal carotid artery. The ratio did not provide a means to estimate the degree of stenosis within high-grade lesions. It is a well known fact that systolic peak velocities rise with increasing stenosis, while previous experience (see 5.2. and 5.4.) has demonstrated that diastolic velocities of the common carotid artery are sensitive to changes in the peripheral resistance ${ }^{35}$. Therefore we choose the ratio of peak systolic velocity in the internal carotid artery to the late diastolic velocity, as measured 500 mseconds after peak systole in the common carotid artery to estimate the severity of disease.

A retrospective analysis of 86 common and internal carotid arteries which had been studied with the Duplex scanner was the basis for this study. All of these 
vessels had been assessed with multiplanar contrast arteriography. The diameter reduction of the internal carotid artery was expressed as has been described previously (see 4.3.4.). A group of 30 normal subjects were studied using the Duplex scanner combined with spectral analysis in an identical fasion to the diseased subjects. These subjects were "persumed" normals in that they had not undergone confirmatory arteriography. Their ages ranged from 29 to 32 years.

\subsubsection{Results.}

The range of velocities found in each group is shown in table XIV. The velocity ranges in the common carotid artery (late diastolic velocity) found in each group from normal to stenosis greater than $90 \%$ diameter reduction are wide, but the values tend to fall with increasing disease in the internal carotid artery. The range of peak systolic velocities in the internal carotid artery is also wide in each group, but the values tend to rise with increasing disease in the internal carotid artery.

\section{Table XIV}

Late diastolic velocity and peak systolic velocity ranges in the commmon carotid and intenal carotid artery (cm/sec).

\begin{tabular}{lcc} 
Carotid Lesion & Common Carotid (Diastole) & Internal Carotid (Systole) \\
\hline Normal & $48-53$ & $30-114$ \\
$10-60 \%$ Stenosis & $22.4-53.9$ & $39-220$ \\
$65-90 \%$ Stenosis & $7.9-23.1$ & $123-282$ \\
Greater than & $7.2-15.4$ & $154-325$ \\
$90 \%$ Stenosis &
\end{tabular}

The variation of the ratio VICA (Velocity Internal Carotid Artery) to VCCA (Velocity Common Carotid Artery) with severity of disease is shown in figure 41.

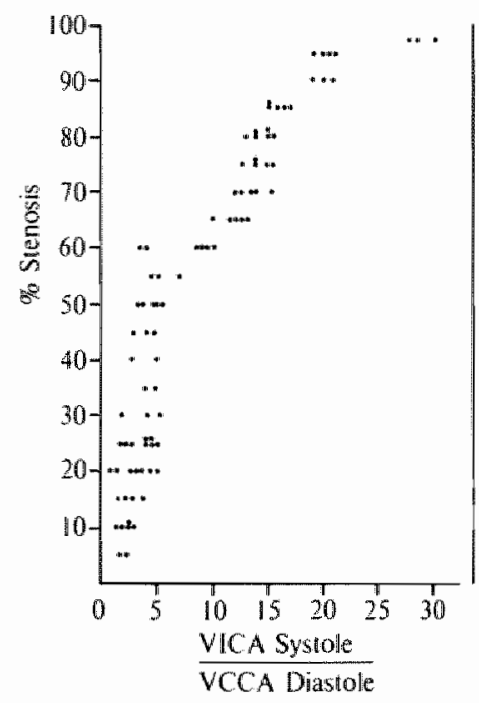

Figure 41

VICA systole / VCCA diastole ratio in the 86 vesse plos pled against the percentage diameter reduction. 
The ratio increases exponentially as the severity of disease increases. The scattergram shows that it is impossible to distinguish disease states less than $60 \%$ using the VICA to VCCA ratio but for stenoses greater than $60 \%$ it is possible by selecting particular values of the ratio to gradate the severity of disease (table $\mathrm{XV}$ ). If the value of 7.5 is selected as defining disease greater than $60 \%$ diameter reduction than $2 / 42(5 \%)$ of the sides are wrongly classified giving an accuracy of $95 \%$. Selecting of a value of 11.0 as defining disease greater than $65 \%$ diameter reduction results in $1 / 36(3 \%)$ of sides being wrongly classified, remaining an accuracy of $97 \%$. Selecting a value of 18.0 allows all diseased vessels with $90 \%$ diameter reduction or more to be correctly classified.

Tulble $\mathrm{XV}$.

Accuracy of VICA/VCCA ratio in predicting disease as compared with contrast arteriography.

\begin{tabular}{lcccc} 
& Category & Number of Sides & Accuracy & Ratio \\
\hline $60-90 \%$ & Stenosis & 42 & $95 \%$ & 7.5 \\
$65-99 \%$ & Stenosis & 36 & $97 \%$ & 11.0 \\
$90-90 \%$ & Stenosis & 10 & $100 \%$ & 18.0 \\
\hline
\end{tabular}

The values of the VICA to VCCA ratio for the normals are shown in figure 42. All were equal to or below 3 with a range from 1.3 to 3 . These did not overlap with the values seen in those with diameter reduction greater than $60 \%$

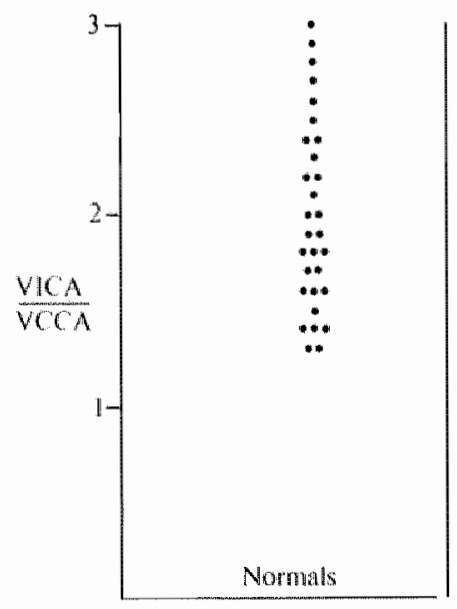

Figure at2.

VICA systole / VCCA diastole ratio for the normals.

The values of the VICA to VCCA ratio for each category of disease have a small range, which for the diseased categories above $60 \%$ diameter reduction do not overlap. There is considerable overlap in values of the VICA to VCCA ratio for all states of disease less than $60 \%$ diameter recluction. 


\subsubsection{Discussion.}

If constant flow rates are to be maintained through a increasing stenosis the welocity of flow through the stenosis must increase (see chapter 3 ). The velocity of flow is proportioned to the cross-sectional area of the arterial lumen at the site of measurement. Studies of steady flow in bifurcating tubes have shown that the mean flow velocity in the branch bears a constant relationship to the velocity in the parent tubes. While the flow velocity is influenced by the cross-sectional area of the vessel, the compliance of the vessel wall and the peripheral resistance also have an effect ${ }^{118}$. The ratio of velocity in the internal carotid artery to the velocity in the common carotid artery will cancel the effects of the compliance of the vessel wall. However, the problem associated with the differing peripheral resistance perfused by the internal and extern carotid artery remains. In a previous report ${ }^{35}$ we implied that changes in the peripheral resistance of the vascular bed of the brain find their reflections in the velocity pattern of the common carotid artery. Since only $20 \%$ of the blood flow in the common carotid artery will supply the external carotid artery, changes in the velocity waveform of the common carotid artery will be in large a reflection of disease in the internal carotid.

Ideally, any velocity ratio, in order to be sensitive to increasing disease, should consist of a numerator which increases with severity of disease and a denominator which decreases with increasing disease. Such a ratio will tend towards infinity with increasing severity of disease. The ratio of peak systolic velocity in the internal carotid artery to the diastolic velocity in the common carotid artery appears to fit this definition.

The present study has demonstrated the feasibility of using a ratio to gradate disease states greater than $60 \%$ diameter reduction. It is not entirely unexpected to find that we were unable to distinguish normals from diseased subjects with diameter reductions less than $60 \%$ using this velocity ratio. Markedly decreased diastolic flow in the common carotid artery is not seen until more severe disease states are reached. The ratio is, therefore, reliant on increasing velocities at peak systole in the internal carotid artery to produce discriminatory changes in the normal to $60 \%$ diameter reduction group. These changes in peak systolic velocity are not seen untill diameter reductions of $50 \%$ or more are present.

When correlating the ratio of velocities with arteriography, it must be expected that there are errors in the classification of disease based on arteriography. Previous described work ${ }^{41}$ has shown that the intraobserver agreement for the classification of carotid disease into five categories was $83 \%$. The interobserver agreement for the same categories was $75 \%$. It is important to bear this variation in mind when interpreting any comparative study. The arteriograms in this series were read by one radiologist; therefore, we do not have to consider the interobserver agreement which is lower than the intraobserver agreement.

While we have used a Duplex scanner to obtain our data, it is possible to use other Doppler devices providing these incorporate a method of measuring the angle of the incident Doppler beam to the long axid of the vessel. A potential problem may arise in cases where the internal carotid artery is kinked and the incident Doppler beam approaches the longitudinal axis of the vessel (see 5.3.3.). 
In conclusion, the algorithm seems to be useful in further gradating the degree of stenosis in the 60-99\% diameter reduction range. It allows the use of a $90-99 \%$ diameter reduction group with an accuracy of 100 percent. 


\section{Chapter 6}

\section{The role of Duplex scanning in clinical practice.}

\subsection{Introduction.}

Noninvasive techniques used in the detection of extracranial carotid artery disease should provide a means of screening patients to select those who should undergo contrast arteriography. Such a noninvasive technique should be able to identify both normal vessels and those with the entire spectrum of disease involvement with an acceptable accuracy. The accuracy of the Duplex concept, as presented in section 5.3., justifies the use of this method as a screenings device. Combining the normals with the $5-15 \%$ diameter reduction category, the agreement between arteriographic and Duplex data is $86 \%$ for the short focus scanhead. In the 50 $99 \%$ diameter reduction category, using the medium focus scanhead, the agreement is $94 \%$. For totall occlusions the agreement was $95 \%$, combining the data from the medium and short focus scanhead.

In addition to the screenings role, Duplex scanning combined with spectral analysis can be used to obtain answers about the prevalence and significance of extracranial carotid artery disease in certain patients populations. An unanswered question relative to the role of noninvasive testing is for example, its impact on the subsequent decision making process by referring physicians. In order to answer this question data of all new referrals in a certain period of time were reviewed. The incidence of extracranial carotid artery disease in patients with asymptomatic bruits and their clinical significance will be presented and discussed in the second study of this chapter. The third study concerns the incidence of extracranial carotid artery disease in patients who are candidates for coronary bypass surgery. The role of a noninvasive test in screening these patients for carotid disease will be discussed. In the last study of this chapter patients were studied prior to and after carotid endarterectomy in order to document velocity pattern changes in the carotid system. The relevance of flow disturbances due to this surgical procedure will be discussed. The four studies in this chapter represent the kind of work performed in a noninvasive vascular laboratory.

\subsection{The influence of ultrasonic Duplex scanning on the management of patients with suspected carotid disease.}

\subsubsection{Introduction.}

A major unanswered question relative to the role of noninvasive testing for carotid artery disease is its impact on the subsequent decision making process by the referring physician. Ideally, only patients who are potential candidates for carotid surgery should undergo contrast arteriography. The role of the noninvasive test is to select those patients who are candidates for carotid surgery 
and avoid contrast arteriography in those who are not $21.58,160$. . While noninvasive testing is often considered to be unnecessary for patients with focal neurological symptoms, there remains a large group of patients with vague neurological symptoms or asymptomatic bruits, in which the outcome of an accurate noninvasive test could be of value to the referring physician.

The referral base for the present study was from a variety of sources both within and outside the Unversity of Washington. The reports sent back to the referring physician contained only the results of the study without any recommendations concerning further diagnostic tests or treatment. In order to assess the impact of the Duplex studies on the management of these patients with possible carotid artery disease, the data of all new referrals between January 1980 and December 1980 were reviewed.

In that period of time 410 patients with neurological symptoms were referred to the vascular laboratory for an ultrasonic evaluation of the extracranial carotid arteries. The patients were grouped according to the reason of referral: (1) patients with focal neurological symptoms $(n=263)$; and $(2)$ patients with nonfocal neurological symptoms $(n=147)$. The patients with focal symptoms were subdivided into those with focal symptoms lasting less than 24 hours $(n=156)$, and those with focal symptoms lasting longer than 24 hours $(n=107)$.

The reports, sent to the referring physicians, contained the results of the study expressed in diameter reductions withoul any further recommendations. At the end of the year the noninvasive data were reviewed and the number of patients in each group that underwent contrast arteriography and subsequent carotid surgery documented.

\subsubsection{Results}

The distribution of carotid artery disease on the appropriate side as estimated by the Duplex scanner in the group of patients with focal neurological symptoms lasting less than 24 hours is shown in table XVI. Using the previously described 5 categories 4 patients were found to have a normal internal carotid artery (category A). A 5-15\% diameter reduction (category B) was found in 64 patients, a $16-49 \%$ diameter reduction (category $\mathrm{C}$ ) was found in 37 patients, a 50 -99\% diameter reduction in 44 patients, and a total occlusion (category E) in 7 patients. Of the patients with a reported $50-99 \%$ diameter reduction of the internal carotid artery (category D), $77 \%(34 / 44)$ underwent contrast arteriography. This was statistically signilicantly different $\left(\mathrm{X}^{2}, \mathrm{p}<0.005\right)$ from the percentages of patients with lesser degrees of disease (25/105) who underwent arteriography. The percentage of patients with a reported total occlusion of the internal carotid artery (category $E$ ) who underwent contrast arteriography was $43 \%$ (3/7).

The percentage of patients who underwent subsequently carotid surgery was $0 \%$ for category A, $21 \%(3 / 14)$ for category B, $20 \%$ (2/10) for category C, $88 \%$ ( $30 \%$ 34 ) for category $\mathrm{D}$ and $0 \%$ for category $\mathrm{E}$. Again the highest percentage was found in the $50-99 \%$ diameter reduction group.

The distribution of carotid disease in patients with focal neurological symptoms lasting longer than 24 hours is shown in table XVII. A normal internal carotid artery was found in one patient, a $5-15 \%$ diameter reduction (category B) in 45 
Duplex categories, number of arteriograms and number of operations in patients with focal neurological symptoms lasting less than 24 hours.

\section{Focal Neurological Symptoms \\ (Less than 24 hours)}

\begin{tabular}{lcccc}
\hline & Duplex & \multicolumn{2}{c}{ Arteriogram } & Surgery \\
\hline Category & Number & Number & $\begin{array}{c}\text { Percentage } \\
\text { of Total }\end{array}$ & Number \\
A & 4 & 1 & $25 \%$ & 0 \\
B & 64 & 14 & $22 \%$ & 3 \\
C & 37 & 10 & $27 \%$ & 2 \\
D & 44 & 34 & $77 \%$ & 30 \\
E & 7 & 3 & $43 \%$ & 0 \\
\hline Total & $\mathbf{1 5 6}$ & $\mathbf{6 2}$ & $40 \%$ & $\mathbf{3 5}$ \\
\hline
\end{tabular}

Table XVIT.

Duplex categories, number of arteriograms and number of operations in patients with focal symptoms lasting longer than 24 hours.

\section{Focal Neurological Symptoms}

(Longer than 24 hours)

\begin{tabular}{lcccc}
\hline & Duplex & \multicolumn{2}{c}{ Arteriogram } & Surgery \\
\hline Category & Number & Number & $\begin{array}{c}\text { Percentage } \\
\text { of Total }\end{array}$ & Number \\
A & 1 & 0 & $0 \%$ & 0 \\
B & 45 & 5 & $11 \%$ & 0 \\
C & 34 & 3 & $9 \%$ & 2 \\
D & 17 & 8 & $47 \%$ & 4 \\
E & 10 & 1 & $10 \%$ & 0 \\
\hline Total & 107 & 17 & $16 \%$ & 6 \\
\hline
\end{tabular}

patients. A 16-49\% diameter reduction (Category C) was found in 34 patients, a 50-99\% diameter reduction (Category D) in 17 patients, and a total occlusion (Category E) in 10 patients. Of the patients with a $50-99 \%$ diameter reduction $47 \%(8 / 17)$ underwent contrast arteriography. This was statistically significantly different $\left(\mathrm{X}^{2}, \mathrm{p}<0.005\right)$ from the percentages of patients with lesser degrees of disease $(8 / 80)$ who underwent contrast arteriography. Of the 10 patients with a 
reported total occlusion of the related internal carotid artery one underwent arteriography (10\%). The percentage of patients who underwent subsequently carotid surgery was $0 \%$ for category $\mathrm{A}, 0 \%$ for category $\mathrm{B}, 67 \%(2 / 3)$ for category $\mathrm{C}, 50 \%$ (4/8) for category $\mathrm{D}$, and $0 \%$ for category $\mathrm{E}$.

The distribution of carotid disease in the group of patients with nonfocal symptoms is shown in tabel XVIII. The side reported in the table is the one with

Table XVIII.

Duplek categories, number of arteriograms and number of operations in patients with nonfocal netrologicall symptoms.

\begin{tabular}{lcccc}
\hline \multicolumn{4}{c}{ Nonfocal Neurological Symptoms } \\
\hline Category & Duplex & Arteriogram & Surgery \\
\hline A & Number & Number & $\begin{array}{c}\text { Percentage } \\
\text { of Total }\end{array}$ & Number \\
B & 0 & 0 & $0 \%$ & 0 \\
C & 48 & 0 & $0 \%$ & 0 \\
D & 70 & 5 & $7 \%$ & 2 \\
E & 25 & 13 & $52 \%$ & 9 \\
\hline Total & 4 & 2 & $50 \%$ & 0 \\
\hline
\end{tabular}

the most marked disease. A normal internal carotid artery was not found in this group of patients, a $5-15 \%$ diameter reduction (category B) was found in 48 paticnts. A $16-49 \%$ diameter reduction (category $C$ ) was found in 70 patients, a $50.99 \%$ diameter reduction (category D) was found in 25 patients, and a total occlusion (category E) in 4 patients. Of the patients with a $50-99 \%$ diameter reduction $52 \%$ (13/25) underwent contrast arteriography. That was statistically signillicantly different $\left(X^{2}, p<0.005\right)$ from the percentages of patients with lesser degrees of disease $(5 / 118)$ who underwent contrast arteriography. Of the patients with a reported total occlusion $50 \%$ (2/4) underwent contrast arteriography. Table XIX shows in a tabulation the distribution of carotid disease on both sides in the group of patients with nonfocal symptoms. The two patients with an occlusion (category $\mathbb{E}$ ) who underwent contrast arteriography, were found to have a $50-99 \%$ diameter reduction (category D) on the contralateral side, which was probably the reason for the arteriography. Of the patients with at least one $50-99 \%$ diameter reduction $75 \%$ (15/20) underwent contrast arteriography.

A tolal of eleven patients underwent carotid surgery in this group; nine of these patients had at least one high grade stenosis $(50-99 \%$ diameter reduction) of the internal carotid artery. Of these nine patients five had a bilateral high grade stenosis and wo had a high grade stenosis with a contralateral total occlusion of the internal carotid artery. 
Table XIX.

Duplex categories of the right and left internal carotid artery in patients witl nonfocal neurologicall symptoms. The right side is displayed on the horizontal and the left side on the vertical axis. The numbers in parentheses are the number of patients who underwent arteriography.

\section{Non focal Neurological Symptoms}

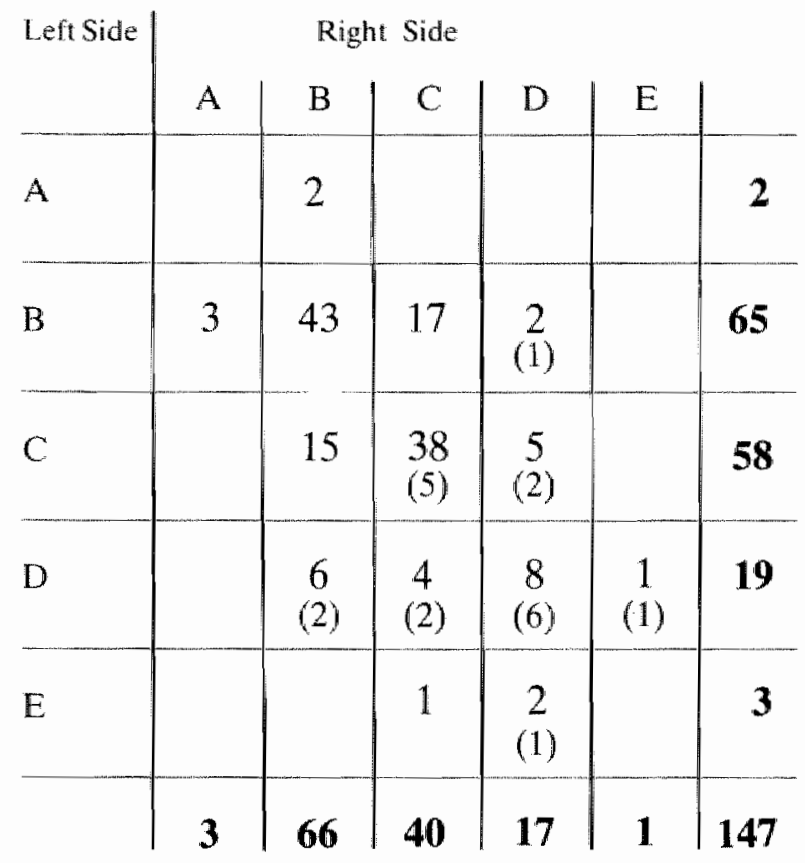

\subsubsection{Discussion.}

The question of the clinical role that noninvasive tests play in the management of patients with suspected carotid disease needs to be answered separately for each group of patients. The role of the noninvasive test in a patient with transient focal neurological symptoms will be different from that in a patient with vague nonfocal neurological symptoms or in a patient with an asymptomatic bruit. This was clearly reflected in the numbers of patients who underwent contrast arteriography in the different groups. As might be expected patients with transient focal symptoms were more likelly to have arteriography than patients in the other groups.

However, a surprising finding was that even though patients were classified as having focal symptoms by the referring physician, there remained a considerable number who did not have arteriography. For the overall group of 263 patients with focal symptoms, $70 \%$ did not have a contrast study. The reasoning behind this approach is unknown, but it would appear that patients with high grade stenoses (category D) were given special consideration with regard to further study. "This is reflected in the fact that $69 \%$ of patients in this category did undergo arteriography. When the symptoms are broken down by duration, in those with 
episodes lasting less than 24 hours the number of arteriograms performed was greatest $(40 \%)$. For categories $B$ and $C$, this was 22 and $27 \%$, the highest for any of the other groups. This would be consistent with current ideas relative to this problem which is usually given highest priority with regards to both diagnosis and management ${ }^{\text {s. }}$.

For patients in the group with focal symptoms lasting less than 24 hours, there was a significant difference $\left(X^{2}, p<0.005\right)$ between the number of patients who underwent surgery in the D category (30/34) and categories B and C. The finding of a high grade stenosis by arteriography was clearly of importance in the decision 10 carry out surgical treatment ${ }^{2 \%}$, $1 \%$. Of the four patients in the D category who did not undergo surgery, the Duplex study reported a high grade stenosis which wis not confirmed by contrast arteriography in two cases. In two cases the general condition of the patient was considered to be a contra-indication for surgery.

The most perplexing group of patients from a clinical standpoint are those with nonfocal symptoms, especially when the clinical picture is vague which is often the case $62,65,119$. In this group, the results were not surprising since if the symptoms are due to extracranial arterial disease, they are often thought to be due to a reduction in cerebral blood flow and not to emboli. In this group $52 \%$ with either a high grade stenosis or occlusion did have arteriography with only $4 \%$ of those with lesser degrees of disease having contrast studies. Of the eleven patients who underwent carotid surgery, nine patients had at least one high grade lesion. The presence of bilaterally significant disease (7/9) seems to have played a role in the decision to proceed with surgical treatment.

It is clearly not possible to draw any firm conclusions concerning the subsequent workup of patients referred to a noninvasive laboratory. However, it is clear that in general physicians (rightly or wrongly) consider the finding of a high grade stenosis of great clinical importance for each of the patient subgroups.

It could well be argued that the studies might have an adverse impact on subsequent management. For example, patients with focal symptoms lasting less than 24 hours are in general considered candidates for anteriography regardless of the results of any noninvasive tests. Yet in this patient subgroup $60 \%$ did not have what many consider to be the definitive study. While many of these patients may not have had the so-called classic symptoms if examined by a single neurologist experienced in the field, this was the basis for referral. It should be noted that it is our own policy to require contrast arteriography in all patients with typical transicnt ischemic attacks or amaurosis fugax (focal symptoms lasting less than 24 hours) regardless the result of the Duplex test.

Regardless of the ultimate answers to many of the questions posed, it is clear that the results of the Duplex test reported to the physician did influence his or her decision to proceed with arteriography. Furthermore, it is also evident that the finding of a high grade stenosis on the arteriogram resulted in a greater number of patients undergoing carotid surgery. Only subsequent follow-up studies will provide the answers to many remaining questions concerning the correct approach in the evaluation and treatment of patients with suspected carotid disease. 


\subsection{The impact of Duplex scanning on the evaluation of patients with asymptomatic bruits.}

\subsubsection{Introduction.}

The finding of a bruit in the neck has been the standard clinical method for the detection of unsuspected disease at the carotid bifurcation. On the other hand is the presence of a bruit in the neck an aspecific finding. The incidence of neck bruits in 1,000 persumed normal subjects from age 4 to age 94 performed by Hammond and Eisinger ${ }^{74}$ was $31 \%$. The incidence of neck bruits varied with age; in $87 \%$ of the subjects under 5 years of age a bruit was found, with advancing age the frequency decreased so that by the age of 60 only $12 \%$ had audible bruits. In a group of 70 medical students who were free from obvious disease Rennie et al. ${ }^{140}$ found neck bruits in $40 \%$ of the cases. If the neck bruits are found with this frequency even in normal subjects, what revelance could this have to the overall problem of carotid artery disease?

Clearly the finding of a bruit is inadequate to predict either its origin or the extent of narrowing of the involved vessel. More information about the extent of carotid disease in patients with neck bruits can be obtained by arteriography or noninvasive studies.

There are several arteriographic studies that have examined the extent of carotid disease in patients with asymptomatic bruits. David et al. ${ }^{44}$ in a series of 417 patients undergoing arteriography reviewed their finding in patients with asymptomatic bruits. The internal carotid artery was normal in $14 \%$ while $47 \%$ had a stenosis which reduced the diameter by $50 \%$ or more. Ziegler et al. ${ }^{185}$ demonstrated that carotid disease can be present without an associated neck bruit. These authors found in a study of 199 patients who underwent arteriography that $73 \%$ had a stenosis without the presence of a bruit. Out of 208 asymptomatic bruits Riles et al. ${ }^{143}$ found arteriographically proven high grade stenoses (60)-99\% diameter reduction) in $61 \%$ of the cases. It is difficult to extrapolate the exact incidence of high grade lesions in patients with asymptomatic bruits from arteriographic studies. These patients were for one reason or the other already selected for an invasive procedure.

Noninvasive tests are used frequently to evaluate the extent of extracranial carotid artery disease in patients with an asymptomatic bruit. Out of the 750 patients evaluated by the Duplex scanner between January 1978 and January 1980,100 patients $(13 \%)$ were referred to the vascular laboratory because of an asymptomatic bruit ${ }^{60}$. Kartchner and McRae ${ }^{\text {gh }}$ using pulse-delay OPG (see 2.2.2.) found $21 \%(273 / 1287)$ positive tests in patients with asymptomatic bruits. Gee et al. ${ }^{69}$ using pressure-OPG (see 2.2.2.) found a positive test in $36 \%(42 / 116)$ of the patients screened because of an asymptomatic bruit. Out of the 165 asymptomatic bruits of the 100 patients in the study of Fell et al $.60,37 \%(61 / 165)$ were found to have a $50-99 \%$ diameter reduction. In $6 \%(9 / 165)$ an asymptomatic bruit was found in combination with a total occlusion of the internal carotid artery.

One other noninvasive test should be mentioned, although not commonly used yet for screening patients with asymptomatic bruits. Duncan et al. ${ }^{52}$ reported the use 
of phonoangiographic spectral analysis (see 2.2.3.). This method has been evaluated at the University of Washington ${ }^{104}$. A good correlation between phonoangiography and contrast arteriography was found in all 43 documentated stenoses of the internal carotid artery. This method, which accurately quantified the residual diameter of the internal carotid artery, seems to be valuable for follow-up studies to document disease progression.

In the present study are reported the results in 95 patients referred to our laboratory between January 1980 and May 1981 because of an asymptomatic bruit in the region of the carotid arteries. All patients had at least an unilateral bruit originating in the mid-cervical region, radiating to the base of the skull. All patients were free of symptoms related to extracranial carotid artery disease.

\subsubsection{Results.}

Of the 95 patients unilateral bruits were found in 48 patients and bilateral bruits in 47 patients. A total of 142 carotid sides with a bruit were available for evaluation. The distribution of disease as estimated by the Duplex scanner combined with spectral analysis is shown in figure 43 . Out of the 142 carotid sides $2 \%(3 / 142)$ were found to be normal (category A). Spectral broadening in the deceleration phase of systole, consistent with a 5-15\% diameter reduction (category B), was found in $30 \%(42 / 142)$. Spectral broadening throughout systole, consistent with a $16-49 \%$ diameter reduction (category $\mathrm{C}$ ), was found in $37 \%$ (53/142). Peak systolic frequencies above $4 \mathrm{KHz}$ with spectral broadening throughout systole and increased diastolic frequencies, consistent with a $50-99 \%$ diameter reduction (category D), was found in $29 \%(41 / 142)$. Finally, $2 \%(3 / 142)$ of the patients were found to have a total occlusion of the internal carotid artery.

\section{Asymtomatic Bruits}

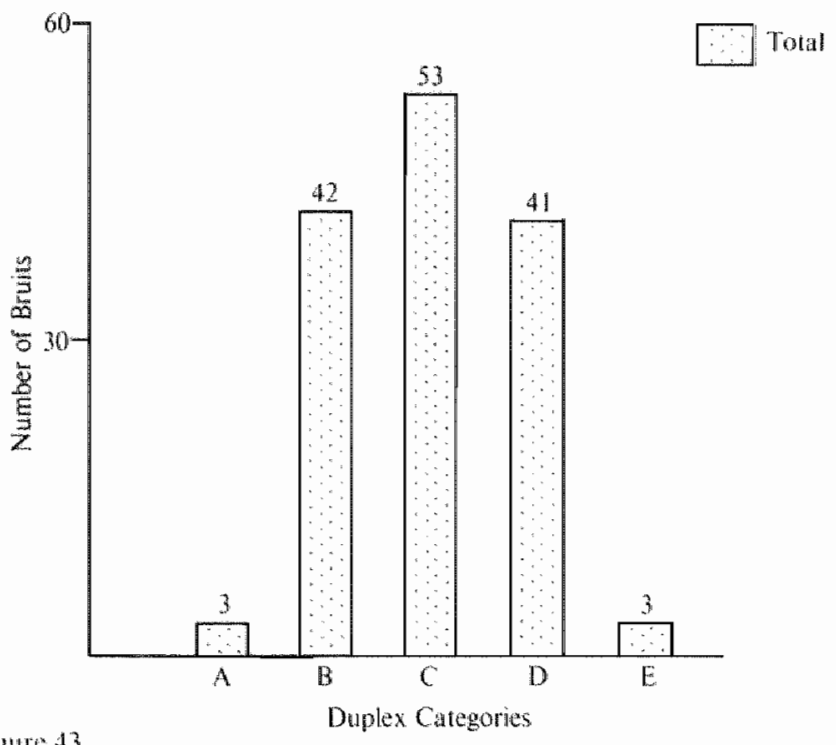

Figure 43.

Histogatn demonstrathg the Duplex category in the 142 carotid sides with a brut 
The distribution of internal carotid artery disease in patients with bilateral bruits is shown in table XX. None of the patients with bilateral bruits were found to have normal internal carotid arteries. There were eleven patients with bilateral diameter reductions of $5-15 \%$ or less. A diameter reduction of less than $50 \%$ was found in $60 \%(28 / 47)$ of the patients with the remaining $40 \%$ (19/47) having a diameter reduction of more than $50 \%$. Bilateral high grade lesions were found in $21 \%(10 / 47)$ of the patients. The asymptomatic occlusions were found with contralateral $50-99 \%$ diameter reduction in two and with a contralateral $5-15 \%$ diameter reduction in one patient. The incidence of high grade lesions (category $D$ and $E$ ) were found in $32 \%$ (15/48) of the patients with unilateral and $21 \%(10 / 47)$ of the patients with bilateral bruits.

\section{Table XX.}

Duplex categories of the right and left internal carotid artery in patients with bilateral bruits. The right side is displayed on the horizontal and the left side on the vertical axis.

\section{Bilateral Bruits}

\begin{tabular}{r|r|r|r|r|r|r} 
L.eft Side & \multirow{2}{*}{$0 \%$} & $5-15 \%$ & $16-49 \%$ & $50-99 \%$ & $100 \%$ & \\
\hline $0 \%$ & & & & & & \\
\hline $5-15 \%$ & 2 & 9 & 2 & 2 & & 15 \\
\hline $16-49 \%$ & & 2 & 13 & & & 15 \\
\hline $50-99 \%$ & & 3 & 3 & 8 & 2 & 16 \\
\hline $100 \%$ & & 1 & & & & 1 \\
\hline & 2 & 15 & 18 & 10 & 2 & 47
\end{tabular}

To evaluate the impact of the Duplex results on the managment of patients with asymptomatic bruits, the data of all new referrals were reviewed. Eighty-one of the 95 patients were new referrals, their data was used for further analysis. Of these 81 patients, $13(16 \%)$ underwent contrast arteriography (see figure 44). 


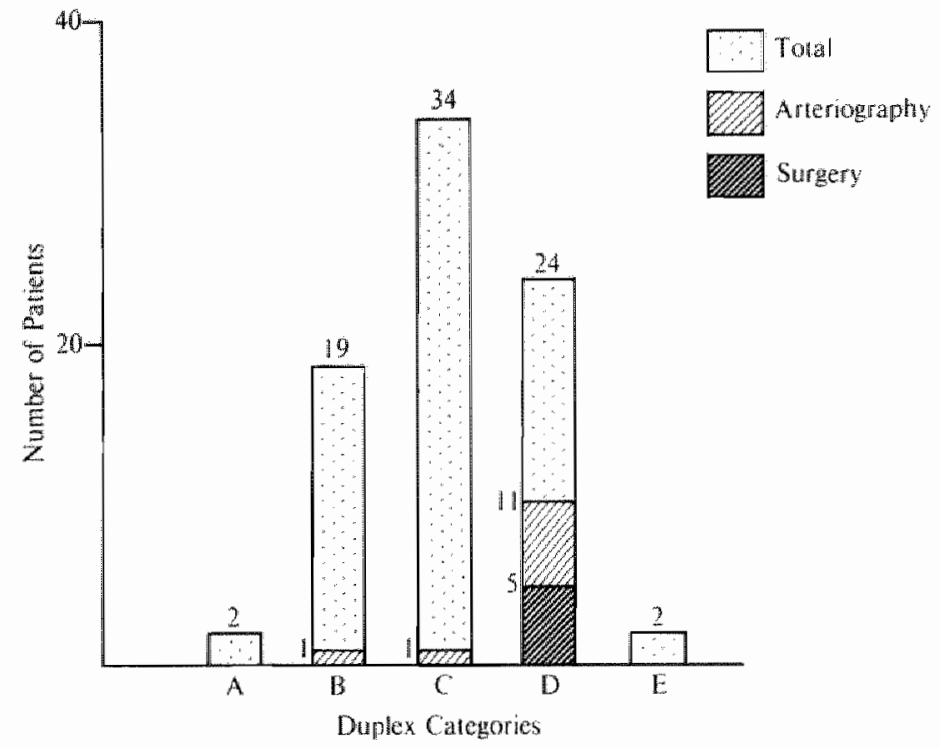

Figure 44.

Histogram demonstrating the Duplex category, the number of arteriograms and the number of operations in the 81 patients with asymptomatic bruits.

Eleven of these patients had high grade lesions with the remaining two having lesser degrees of involvement. Five patients underwent unilateral carotid endarterectomy, and in each case bilateral high grade stenoses were present.

\subsubsection{Discussion.}

The prevalence of high grade stenoses of the internal carotid artery in patients with asymptomatic bruits as documentated with noninvasive tests varies from $21 \%$ $1037 \%$ 60.\% . The prevalence of the high grade lesions $(50-99 \%$ diameter reductions and total occlusions) in the present study was $31 \%$ (44/142), which is comparable with the other studies. Bilateral bruits were not associated with more arteriosclerotic disease than when bruits were found only on one side.

A major point of interest and controversy is the management of patients with asymptomatic bruits when arteriosclerotic disease is found. The policy of the referring physicians, sending their symptomatic patients to the vascular laboratory at the University of Washington has been discussed previously $(6,2.3$.). These physicians receive a report of the study findings expressed in diameter reduction of the internal carotid artery without any recommendations concerning either arteriography or operation. In the group of 81 new referrals with asymptomatic bruits $84 \%$ did not undergo further study. Of the 24 patients with high grade stenoses $46 \%$ (11/24) underwent arteriography. Of the five patients who underwent carotid endarterectomy, all had bilateral high grade stenoses. It appears that, rightly or wrongly, high grade stenoses were considered of clinical importance and in that respect influenced the subsequent workup and therapy of the patients. If physicians consider high grade lesions as the basis for further 
treatment, there is an significant role for a test such as Duplex scanning. Of the 95 patients in the present study $29 \%$ were found to a $50-99 \%$ diameter reduction. If such a lesion is an indication for a more aggressive approach $71 \%$ of the patients would not be investigated further.

Unfortunately a review of the literature does not provide conclusive guidelines relative to this important clinical problem. However, it is becoming clear that the presence of a bruit in and of itself is of little help in predicting the site or extent of disease producing the finding. Ziegler ${ }^{185}$ demonstrated with contrast arteriography that severe disease can be presented without bruits, David ${ }^{4 y}$ and Riles ${ }^{143}$ found an incidence of $47 \%$ and $61 \%$ respectively of high grade lesions in their arteriographic studies.

Given the obvious fallibility of the cervical bruit as an indicator of carotid disease, what is the subsequent course of patients followed without the benefit of accurate definition of disease at the carotid bifurcation? Heyman et al. ${ }^{82}$ reported a 5 to 7 year follow-up of 72 patients with asymptomatic cervical bruits in a rural southern community. They noted that the prevalence of bruits increased with age, was greater in females and in patients with hypertension. The striking finding was that the presence of a bruit was associated with a higher risk of stroke in males but not in females. Furthermore, the side of the bruit and the side of stroke were poorly correlated. In other words, male patients with asymptomatic bruits have a higher risk of stroke, but the side of the bruit does not predict the side of the stroke.

In the Framingham study Wolf et al. ${ }^{182}$ found asymptomatic bruits in 171 patients. In 8 years of follow-up these patients were found to be at higher risk for myocardial infarcation and stroke. More often than not, cerebral infarction occured in a vascular territory different from that of the carotid bruit. The authors concluded that a carotid bruit is clearly an indicator of increased stroke risk but also a sign of advanced general arteriosclerosis involving such major vascular beds as the coronary circulation.

Humphries, et al. ${ }^{89}$ retrospectively analyzed 168 patients (182 arteries) with arteriographically confirmed stenoses of the internal carotid artery equal to or greater than $50 \%$ diameter reduction, who had no symptoms referable to that vessel. Of the 168 patients, 111 had undergone carotid endarterectomy on the opposite side. Of these patients followed an average of 32 months, 26 developed transient ischemic attacks, all of whom underwent successful endarterectomy. Three developed symptoms which were ignored and subsequently went on to a completed stroke. Only one patient sustained a stroke without warning symptoms. No information was provided on patients with lesser degrees of stenosis. The authors suggested that surgery was not reguired for the asymptomatic patient until transient ischemic attacks develop.

A contrasting view is that presented by Thompson's group ${ }^{167,170,}$, who advocate operating upon asymptomatic lesions which are "high grade" in nature. As stated by these authors, the major difficulty is related to identifying which patients should be investigated further with arteriography.

The approach at the University of Washington to the problem has been largerly conservative both from the standpoint of arteriography and surgery. Even in patients who are candiates for major cardiovascular surgery, there is no firm 
evidence that prophyllactic carotid surgery is indicated ${ }^{14.33}$. We are presently comfortable with the current approach to screen patients as described for the following reasons: (1) the accuracy of the test as compared with contrast arteriography is sufficiently good to identify the location and extent of the involvement; $(2)$ once patients with disease are identified, it would appear prudent to follow them closely with repeat studies to document the natural history of the lesions; and (3) it is probably the only way prospective studies can be done which may answer many of the current questions.

Even given all uncertainties about the management of these patients, it is evident that the linding of a high grade lesion influenced the judgement of referring physicians. It appears that these patients for reasons yet unclear are considered to be more "stroke prone", although evidence for this view is not yet available. The question which needs to be answered is whether this is in fact the case.

To answer this question will require prospective studies to determine which lesions do in fact place the asymptomatic patient at risk. If emboli are the most important cause of stroke, then the degree of stenosis may bear no relevance to the clinical course of the patient. It is now clear that the necessary methods are currentlly available to carry out such a prospective study.

\subsection{Carotid arterial disease in patients undergoing coronary bypass surgery.}

\subsection{Introduction.}

The low incidence of transient and permanent complications following carotid thromboendarterectomy for symptomatic and asymptomatic carotid artery disease has led many authors to advocate this procedure as a measure to prevent stroke associated with coronary bypass surgery $46.58,81$. The incidence of postoperative stroke following coronary bypass surgery, however, is relatively low ${ }^{2.141}$. This suggests that patients with symptomatic ischemic coronary artery disease do not have a high incidence of significant carotid artery disease, or that in the group of patients undergoing coronary artery bypass surgery significant carotid arterial disease is not a major risk factor in the pathogenesis of postoperative stroke.

A prospective study with the ultrasonic Duplex scanner combined with spectral analysis as described in chapter 4 was undertaken to document the prevalence of extracranial catotid artery disease in patients undergoing coronary artery bypass surgery and to evaluate the practical use of this noninvasive method to screen patients preoperatively.

From January 1980 to July 1980,102 patients who underwent coronary artery bypass surgery at the University Hospital, Seattle, were studied prior to operation. Of these patients, $23 \%$ (24/102) were referred for evaluation due to the presence of symptoms or signs related to carotid arterial disease (requested group); $77 \%$ (78/102) were evaluated as part of a study protocol (unrequested group) in which the result of the study was not reported to the patient"s physician. 


\subsubsection{Results.}

In the requested group, 14 patients ( $58 \%$ ) were sent to the laboratory because of an asymptomatic bruit in the region of the carotid arteries. Ten patients $(42 \%)$ were sent to the laboratory because the history revealed symptoms possibly related to extracranial carotid artery disease. The mean age of the patients, the male-female ratio, the incidence of a positive history for hypertension, diabetes mellitus and the use of cigarettes are shown in table XXI.

Table XXI.

Risk factors for extracramial carotid artery disease in the requested and unrequested group.

\begin{tabular}{lcc}
\hline & $\begin{array}{c}\text { Requested } \\
(\mathrm{n}=24)\end{array}$ & $\begin{array}{c}\text { Unrequested } \\
(\mathrm{n}=78)\end{array}$ \\
\hline Mean age & 61.0 & 57.7 \\
Male-female ratio & $4: 1$ & $9: 1$ \\
Hypertension & $38 \%$ & $44 \%$ \\
Diabetes Mellitus & $12.5 \%$ & $10 \%$ \\
Smoking history & $97.5 \%$ & $70 \%$ \\
\hline
\end{tabular}

The incidence of unstable angina, a cardiac risk factor for peri- operative cardiac complications for the requested and unrequested group is shown in table XXII. A neck bruit was present in 21 patient in the requested group, in eight of these bilateral bruits were noted. In the unrequested group four patients were found to have a cervical bruit when examined in the vascular laboratory.

Table XXII.

Prevalence of cardiac risk factors in the requested and unrequested group.

\begin{tabular}{lcc}
\hline & $\begin{array}{c}\text { Requested } \\
(n=24)\end{array}$ & $\begin{array}{c}\text { Unrequested } \\
(\mathrm{n}=78)\end{array}$ \\
\hline Chronic angina & 15 & 38 \\
Angina at rest & 7 & 25 \\
Unstabel angina & 2 & 16 \\
\hline
\end{tabular}

A history of a recent focal transient ischemic attack was noted in three patients in the requested group. One patient in the unrequested group had experienced a transient ischemic attack approximately 10 years prior to the study with another sustaining a hemispheric stroke more than 10 years prior to study with complete neurological recovery. In the requested group five carotid endarterectomies were performed; two symchronously and three prior to the coronary bypass procedure. The prevalence and extent of carotid artery disease as estimated by the Duplex scanner combined with spectral analysis in the unrequested group is shown in 
table XXIII. In this group five patients had unilateral or bilateral stenoses of more than $50 \%$ diameter reduction (category D) in the internal carotid artery, and 50 patients ( $64 \%$ ) had no or minimal disease (category $\mathrm{A}$ or $\mathrm{B}$ ).

Tablie XXIII.

Duplex categories of the right and left internal carotid antery in the unrequested group. The right side is displayed on the vertical and the left side on the horizontal axis.

\section{Unrequested}

\begin{tabular}{l|c|c|c|c|c}
$\begin{array}{l}\text { Right } \\
\text { Internal } \\
\text { Artery }\end{array}$ & \multicolumn{3}{|c|}{ Left Internal Carotid Artery } \\
\hline A & B & C & D & Total \\
\hline A & 2 & 8 & & & 10 \\
\hline B & 3 & 37 & 12 & 1 & 53 \\
\hline C & 1 & 5 & 5 & 2 & 13 \\
\hline D & & & & 2 & 2 \\
\hline Total & 6 & 50 & 17 & 5 & 78 \\
\hline
\end{tabular}

The prevalence and extent of carotid disease as estimated by the Duplex scanner combined with spectral analysis in the requested is shown in table XXIV. In this

Table XXIV.

Duplex categories of the right and left internal carotid artery in the requested group. The right side is displayed on the vertical and the left side on the horizontal axis.

\section{Requested}

\begin{tabular}{|c|c|c|c|c|c|c|}
\hline \multirow{2}{*}{$\begin{array}{l}\text { Right } \\
\text { Internal } \\
\text { Calrotid } \\
\text { Artery }\end{array}$} & \multicolumn{6}{|c|}{ Left Internal Carotid Artery } \\
\hline & A & $\mathrm{B}$ & $\mathrm{C}$ & $\mathrm{D}$ & $\mathrm{E}$ & Total \\
\hline A & & 1 & & & & 1 \\
\hline $\mathrm{B}$ & & 2 & & & & 2 \\
\hline $\mathrm{C}$ & & 1 & 7 & 4 & 1 & 13 \\
\hline $\mathrm{D}$ & & & 3 & 4 & 1 & 8 \\
\hline $\mathrm{E}$ & & & & & & 0 \\
\hline Total & 0 & 4 & 10 & 8 & 2 & 24 \\
\hline
\end{tabular}


group, thirteen patients had at least one stenosis of more than $50 \%$ diameter reduction (category $D$ and $E$ ) and three patients $(13 \%)$ had no or minimal disease (category $\mathrm{A}$ or $\mathrm{B}$ ).

One postoperative stroke occured in a 49 years old male with a $16-49 \%$ diameter reduction (category $\mathrm{C}$ ) of the appropriate internal carotid artery. One postoperative transient ischemic attack occured in a 50 years old male with a 16 $49 \%$ diameter reduction (category $\mathrm{C}$ ) of the related internal carotid artery. Both of these were in patients in the unrequested group.

\subsubsection{Discussion.}

The incidence of postoperative stroke after myocardial revasculation procedures was $2 \%$ in the 1,287 patients reported by Reul, et al. ${ }^{141}$ and Ashor et al. ${ }^{2}$ also reported a $2 \%$ stroke rate in 100 patients aged 65 years of more. Although the postoperative stroke rate is relatively low, the preoperative detection of carotid arterial disease both by invasive and noninvasive methods is advocated by many authors. It is less clear as to what the subsequent management of the patient should be, when concomitant carotid arterial disease is found in patients awaiting coronary artery bypass grafts for angina pectoris.

Carotid endarterectomy either prior to or combined with coronary bypass procedures have been advocated by many authors ${ }^{46,58,81}$ both for symptomatic and asymptomatic patients. However, for the asymptomatic patient it is usually not stated how extensive the carotid disease needs to be before endarterectomy is undertaken. Others have remained skeptical of the relationship between asymptomatic carotid occlusive disease and the incidence of perioperative stroke. Barnes, et al. ${ }^{14}$ found an incidence of $16 \%$ of severe carotid disease $150 \%$ or greater diameter reduction) in 102 patients who were candidates for major cardiovascular surgery. However, there were no perioperative strokes or transient ischemic attacks in this group of patients. In a follow-up study of these patients ${ }^{16}$ Barnes noted that with severe carotid disease diagnosed by the noninvasive test patients had a greater chance of developing a myocardial infarction. In 330 patients screened by Doppler imaging and spectral analysis prior to cardiovascular surgery, Turnipseed, et al. ${ }^{174}$ noted no relation between the severity of carotid artery disease and the incidence of perioperative stroke.

In the present study the risk factors for neurological complications in the two group were comparable. In the un requested group the cardiac risk factor (unstable angina) was found more frequently (16/78) than in the requested group $(2 / 24)$. In the unrequested group, only $6 \%(5 / 78)$ had a stenosis of $50 \%$ diameter reduction or more of the internal carotid artery. In contrast, 54\% (13/24) of the requested group had stenosis of $50 \%$ diameter reduction or more.

Of these thirteen patients with high grade lesions, five underwent carotid. endarterectomy without focal neurological perioperative complications. The remaining eight patients with stenosis of $50 \%$ diameter reduction or more were patients with asymptomatic bruits, and were not considered candidates for carotid surgery by their physician. None of these eight patients had perioperative focal neurological complications. 
The 49 year old male, who sustained a stroke related to the right cerebral hemisphere following coronary bypass surgery, had a $16-49 \%$ diameter reduction of the right and a $5-15 \%$ diameter reduction of the left internal carotid artery. The postoperative transient ischemic attack, related to the right hemisphere occured in a 50 year old male with $16-49 \%$ diameter reduction noted bilaterally in the internal carotid arteries. In both patients who sustained perioperative focal neurological complications, the extent and severity of their carotid disease was established with the noninvasive test. However, in neither case was the degree of stenosis noted by Duplex scanning severe enough to suggest that these two patients were seriously at risk for stroke. One of the explanations could be that most perioperative neurological complications are caused by embolic events, coming from ulcerative plaques. Both arteriography and noninvasive test are not capable of identifying ulcerative plaques accurately.

The results reported in this study suggest that patients with symptoms or signs are more likely to have severe carotid disease as compared to asymptomatic patients awaiting coronary bypass surgery. However, like Barnes ${ }^{14,16}$ and Turnipseed ${ }^{174}$, we found that preoperative noninvasive studies fail to predict which patients among those asymptomatic for carotid disease are at high risk for focal neurological perioperative complications. Adding the three studies together in a population of over 500 patients the incidence of perioperative strokes could not be related to the presence of high grade stenosis as detected by noninvasive tests.

Based upon our study and others, we no longer advocate arteriography prior to coronary bypass surgery even if high grade stenoses are found in patients who do not have a history of focal neurological symptoms. This does not mean, of course, that the patients with suspected carotid disease should not be screened. While the natural history of asymptomatic high grade lesions is as yet unknown it would appear wise to at least counsel both the patient and referring physician that disease is present and continued regular follow-up is to be carried out.

\subsection{Velocity pattern changes following carotid endarterectomy.}

\subsubsection{Introduction.}

The evolution of the role of surgery in the management of cerebrovascular insufficiency spans a short period of 25 years. Although the indications for prophylactic carotid surgery are still controversial (see 6.2.3 and 6.3.3), surgery has become one of the accepted forms of treatment for patients with focal symptoms secondary to extracranial carotid artery disease ${ }^{29,62,169}$. The American experience suggests that the complication rate of carotid endarterectomy ranges from less than $1 \%$ among experienced, skilled surgeons to as high as $15 \%$ when performed by the occasional vascular surgeon ${ }^{171}$. Given these reports it is essential that each surgeon assess his or her own results when considering the place of carotid surgery.

Although the clinical results of carotid surgery are reasonably well documented, little is known about the hemodynamic changes that follow the surgical procedure. While the incidence of recurrent symptoms appears to be low in most reported 
series, the actual rate of restenosis is unk nown since arteriography is only performed when the patient develops recurrent symptoms.

The development of noninvasive methods have made it possible to evaluate the carotid bifurcation after operation. Turnipseed et al, " 773 . using ullrasonic arteriography combined with spectral analysis, found restenoses of the proximal internal carotid artery in $9 \%$ of patients in a 3 year follow-up study. The authors concluded that asymptomatic postoperative internal carotid restenosis is more prevalent than generally reported. Kremen et al. ${ }^{106}$, using pressure oculoplethysmography (see 2.2 .2 .), found a recurrent rate of postoperative stenoses of $9 \%$. Bodily et al. ${ }^{30}$, using Duplex scanning combined with spectral analysis, found flow abnormalities in $49 \%$ of the arteries after carotid surgery. At the University of Washington patients undergoing carotid surgery are routinely studied pre and postoperatively following a strict study protocol. The purpose of the present study was to evaluate the sequential changes that occurred in the velocity patterns of 88 patients who had undergone carotid surgery.

All patients of the study were studied with the Duplex scanner prior to carotid surgery. After carotid surgery the patients were studied at three, six, twelve and iwenty-four months postoperatively. Although not all patients were seen at three months follow-up, all 88 patients were seen atleast once within six months. Sixtyfour patients (69 sides) were studied at three months, 56 patients ( 60 sides) at six months, 54 patients ( 58 sides) at 12 months, and 27 patients ( 29 sides) at twentyfour months after operation.

\subsubsection{Results.}

The distribution of internal carotid artery disease as classified by the velocity patterns and confirmed by contrast arteriography prior to surgery is shown in table XXV. Four percent (3/69) were found to have a $5-15 \%$ diameter reduction

Taline XXV.

Duplex categorics preoperative and three monulhs postoperative of $6^{6}$ internal catorid arteries.

\begin{tabular}{|c|c|c|c|c|c|c|}
\hline & \multirow{2}{*}{$\begin{array}{c}\text { Duplex Category } \\
\text { Preoperative } \\
\begin{array}{c}\text { Number of Operated } \\
\text { Sides }\end{array}\end{array}$} & \multicolumn{5}{|c|}{$\begin{array}{l}\text { Duplex Category } \\
3 \text { Months Postoperative }\end{array}$} \\
\hline & & $\mathrm{A}$ & $\mathbb{B}$ & $\mathrm{C}$ & $D$ & $\mathrm{E}$ \\
\hline \multicolumn{7}{|l|}{ A } \\
\hline B & 3 & & 2 & 1 & & \\
\hline $\mathrm{C}$ & 8 & & 5 & 3 & & \\
\hline $\mathrm{D}$ & 58 & & 20 & 32 & 6 & \\
\hline$E$ & & & & & & \\
\hline
\end{tabular}


(category B), 12\% (8/69) were found to have a $16-49 \%$ diameter reduction (category C). A $50-99 \%$ diameter reduction was found in $84 \%(58 / 69)$. For obvious reasons their were no normals or total occlusions in the group of patients who were candidates for carotid endarterectomy.

Of those sicles with a $\mathrm{B}$ lesion prior to surgery, two sides were found to have spectral broadening in the deceleration phase of systole (B category) 3 months after surgery, one side showed spectral broadening throughout systole (category C). Of the sides classified as $C$ prior to surgery, five sides were found to have spectral broadening in the deceleration phase of systole (category B), three sides were found to have spectral broadening throughout systole (category $C$ ). Of the 58 preoperative $\mathrm{D}$ lesions, 20 sides had spectral broadening in the deceleration phase of systole (category B), 32 sides had spectral broadening throughout systole (category C), and six sides had systolic peak frequencies above $4 \mathrm{KHz}$ with spectral broadening throughout systole (category $\mathrm{D}$ ).

The results of the 6 months follow-up studies are shown in table XXVI. Of those two classified as B prior to surgery, both were found to have spectral broadening in the deceleration phase of sytole. Of the three preoperative internal carotid

Trable XXVI.

Duplex categories preoperative and six months postoperative of 60 internal carotid arteries.

\begin{tabular}{cc|cccc}
\hline & $\begin{array}{c}\text { Duplex Category } \\
\text { Preoperative }\end{array}$ & \multicolumn{4}{c}{$\begin{array}{c}\text { Duplex Category } \\
\text { 6 Months postoperative }\end{array}$} \\
\hline $\begin{array}{c}\text { Number of Operated } \\
\text { Sides }\end{array}$ & A & B & C & D & E \\
\hline A & & & & & \\
\hline B & 2 & & 2 & & \\
\hline C & 3 & & 1 & 2 & \\
\hline D & 55 & & 10 & 34 & 11 \\
\hline E & & & & & \\
\hline
\end{tabular}

arteries classified as $\mathrm{C}$, one was found to have spectral broadening in the deceleration phase of systole (category $\mathrm{B}$ ) and two were found to have spectral broadening throughout systole (category $\mathrm{C}$ ). Of the patients with $\mathrm{B}$ or $\mathrm{C}$ lesions prior to surgery, none was found with a $\mathrm{D}$ velocity pattern after 6 months. Of the 55 sides with a D classification prior to surgery, ten sides had spectral broadening in the deceleration phase of systole (category B) after surgery, 34 sides had spectral broadening throughout systole (category $\mathrm{C}$ ), and eleven sides had systolic peak frequencies well above $4 \mathrm{KHz}$ with spectral broadening throughout systole. The results of the 12 months follow-up studly are shown in table XXVII. Of the two internal carotid arteries classified as B prior to surgery, both were found to 
Duplex categories preoperative and welve months postoperative of 58 intemal carotid arteries.

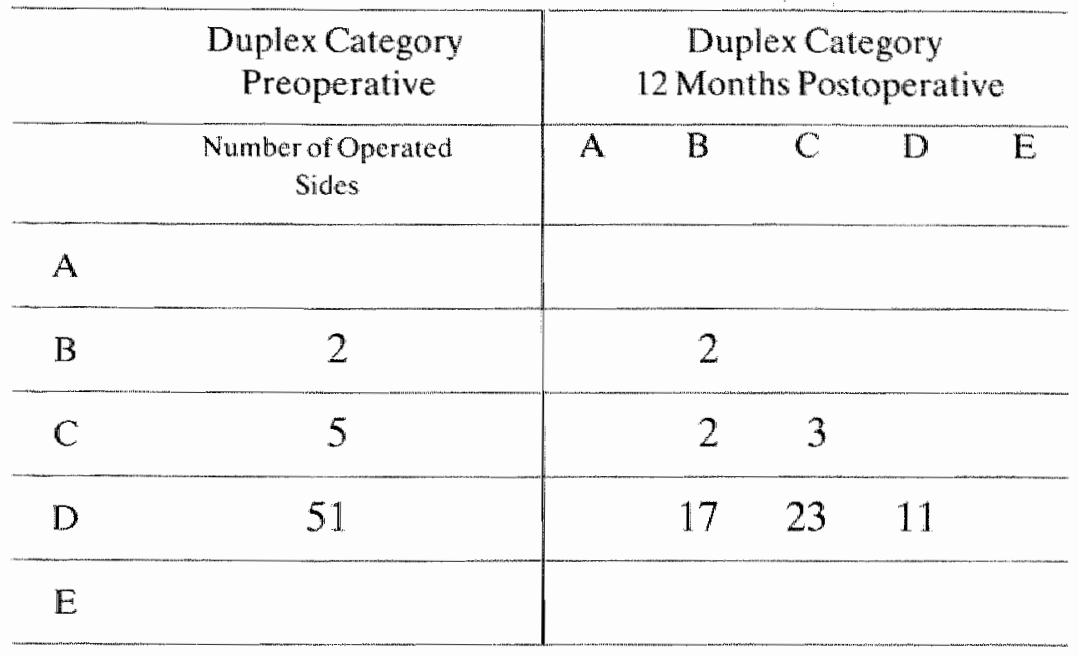

have spectral broadening in the deceleration phase of systole (category $\mathrm{B}$ ). Of the five patients having a $\mathrm{C}$ classification of the internal carotid artery prior to operation, two were found to have spectral broadening in the deceleration phase of systole (category $\mathrm{B}$ ) and three were found to have spectral broadening throughout systole (category $\mathrm{C}$ ) in the 12 months follow-up study. None of the patients with a $\mathrm{B}$ or $\mathrm{C}$ category of the internal carotid artery developed a velocity pattern with systole peak frequencies above $4 \mathrm{KHz}$ and spectral broadening (category D).

Of the 51 sides which were classified as D lesions preoperatively, 17 sides had spectral broadening in the deceleration phase of systole, 23 sides had spectral broadening throughout systole, and eleven sides had systolic peak frequencies well above $4 \mathrm{KHz}$ with spectral broadening after 12 months follow-up (table XVII).

After 24 months follow-up 29 sides were studied (table XVIII). One B categorized internal carotid artery prior to surgery was found to have spectral broadening in the deceleration phase of systole after 24 months follow-up. Of the four sides categorized as $\mathrm{C}$ prior to surgery, one was found to have spectral broadening in the deceleration phase of systole (category $B$ ) and three were found to have spectral broadening throughout systole (category $C$ ). Of the 24 sides with a D categorized internal carotid artery prior to surgery, four sides had spectral broadening in the deceleration phase of systole (category B), 15 sides had spectral broadening throughout systole (category $\mathrm{C}$ ), four sides had systolic peak frequencies well above $4 \mathrm{KHz}$ with spectral broadening (category $\mathrm{D}$ ), and one side was occluded (category E). Again of the sides with a B or C lesion prior to surgery, none developed high systolic peak frequencies in combination with spectral broadening. 
Duplex categories preoperative and 24 months postoperative of 29 intemal carotid arteries.

\begin{tabular}{|c|c|c|c|c|c|c|}
\hline & \multirow{2}{*}{$\begin{array}{c}\begin{array}{c}\text { Duplex Category } \\
\text { Preoperative }\end{array} \\
\begin{array}{c}\text { Number of Operated } \\
\text { Sides }\end{array}\end{array}$} & \multicolumn{5}{|c|}{$\begin{array}{c}\text { Duplex Category } \\
24 \text { Months Postoperative }\end{array}$} \\
\hline & & A & $\mathrm{B}$ & $\mathrm{C}$ & $\mathrm{D}$ & $\mathrm{E}$ \\
\hline \multicolumn{7}{|l|}{ A } \\
\hline$B$ & $\mathbb{1}$ & & 1 & & & \\
\hline C & 4 & & 1 & 3 & & \\
\hline $\mathrm{D}$ & 24 & & 4 & 15 & 4 & 1 \\
\hline $\mathrm{E}$ & & & & & & \\
\hline
\end{tabular}

In the last three years seven patients have undergone contrast arteriography after carotid endarterectomy. Four of these patients are in the present follow-up protocol, with the three remaining not studied preoperatively by the Duplex scanner and therefore not in the present follow-up protocol. The diameter reduction as estimated by Duplex scanning and contrast arteriography are shown in table XXIX.

Table XXIX.

Diameter reduction estimated by contrast arteriography and Duplex scanning in patients after surgery.

\begin{tabular}{lcc}
\hline $\begin{array}{l}\text { Number of } \\
\text { Patients }\end{array}$ & $\begin{array}{c}\text { Arteriographic } \\
\text { Diameter Reduction }\end{array}$ & $\begin{array}{c}\text { Duplex Scanning } \\
\text { Diameter Reduction }\end{array}$ \\
\hline 1 & $20 \%$ & $5-15 \%$ \\
2 & $60 \%$ & $50-99 \%$ \\
$3^{*}$ & $30 \%$ & $16-49 \%$ \\
$4^{*}$ & $45 \%$ & $50-99 \%$ \\
$5^{*}$ & $10 \%$ & $5-15 \%$ \\
6 & $90 \%$ & $50-99 \%$ \\
$7^{*}$ & $20 \%$ & $16-49 \%$ \\
\hline
\end{tabular}

* Patients in present follow-up study.

Five patients demonstrated recurrent symptoms related to the operated sides, two patients developed symptoms related to the contralateral side. Amaurosis fugax related to the operated side was found in one patient with a $B$ velocity pattern and 
in one patient with a $C$ velocity pattern. Motor weakness related to the operated side was found in two patients both with a $C$ velocity pattern. One frank stroke related to the operated side occurred in a patient with a $B$ velocity pattern. Of the two patients with symptoms not related to the operated side one patient with a $C$ lesion on that side sustained a frank stroke, the other developed amaurosis fugax with a $D$ velocity pattern on that side.

\subsubsection{Discussion.}

In order to interpret the velocity patterns from the carotid bifurcation after operation, it is important to review the changes in the arterial segment that are known to occur. The endarterectomized area in time will be covered by proliferation of myointimal cells ${ }^{67}$. Early postoperative arteriograms often show wall irregularities as well as some dilatation of the carotid bulb $50,1,50$. Schutz et at. 150 in a series of 50 arteriograms reported wall irregularities in 12 and local dilatation of the bulb in 6 instances. The wall irregularities appeared to decrease with time, while the dilatation persisted. Diaz et al. ${ }^{50}$ found local dilatation and circumferential defects in the majority of 50 early postoperative arteriograms. The circumferential defects disappeared in subsequent arteriograms. In the present series of 88 patients four underwent arteriography after operation, in two cases dilatation of the bulb was found, and in all four wall irregularities were present. The fact that a normal velocity pattern is not commonly found in our follow-up studies is not surprising since flow disturbances can occur with minimal changes in the intimal surface. In all follow-up studies spectral broadening throughout systole (category C) was found most frequently followed by spectral broadening in the deceleration phase of systole (category B). Both the B and C category can be considered as postoperative findings not related to a recurrent high grade stenosis.

Whenever systolic peak frequencies were noted above $4 \mathrm{KHz}$ with spectral broadening throughout systole (category D), the velocity pattern was classified as consistent with a high grade stenosis $(50-99 \%$ diameter reduction). This was found in $9 \%$ after 3 months, $18 \%$ after 6 months, $19 \%$ after 12 months, and $17 \%$ after 24 months follow-up.

Of interest is the fact that of the 6 sides where a $D$ velocity pattern was noted after three months, in four sides the classification changed to a $\mathrm{C}$ velocity pattern after 12 months. Of the eleven sides where a $D$ velocity pattern was found after 6 months, in five instances a $\mathrm{C}$ velocity pattern was noted after 24 months. These findings suggest that there are changes in the carotid bulb in these patients, similar to those described by Schutz ${ }^{150}$ and Diaz ${ }^{50}$ in their arteriographic studies. Our findings are consistent with the thesis that myointimal profileration can occur early after operation but in some patients may regress with time.

An important question is whether the changes found by Duplex scanning combined with spectral analysis are in lact real or due to errors in our study technique. The latter is very unlikely, based upon our current experience with the Duplex scanner. Another important question relates to the accuracy of the velocity patterns seen after operation in predicting the degree of involvement. As noted in table XXIX, the results in the seven patients who underwent repeated arteriography agreed quite well. Thus, this method of study is not only suitable for 
screening patients with suspected extracranial carotid artery disease but is also useful in predicting the state of the carotid bulb in patients after carotid endarterectomy.

Symptomatic recurrent stenosis after carotid endarterectomy is reported to occur in 1-4 percent of patients ${ }^{45,67,155}$. Asymptomatic recurrent stenosis, as detected by noninvasive tests, are reported in approximately $10 \%{ }^{106,173}$. Reasons for restenoses, except for technical errors, are mainly caused by myointimal hyperplasia or recurrent arteriosclerosis ${ }^{45,67}$. Myointimal hyperplasia is expected within the first 24 months after surgery, whereas arteriosclerotic changes as cause for recurrent stenosis is expected after a longer postoperative period. In the present study, approximately $20 \%$ of the patients developed velocity patterns consistent with a high grade stenosis. However, the development of such flow patterns was not associated with recurrent of symptoms in the majority of these patients. This is not surprising since most of the early lesions which develop are smooth and do not ulcerate and do not become potential sites for embolization. Also, as indicated above, there is evidence that with time, these velocity patterns will change in some patients towards a more normal range.

The present study has demonstrated that the Duplex scanner combined with spectral analysis is a reliable method to follow patients after carotid endarterectomy. The study shows that approximately $20 \%$ of the operated sides develop a velocity pattern consistent with a $50-99 \%$ diameter reduction. The number of patients and follow-up time in this study is not large or long enough to draw definitive conclusions. It is clear however, that a noninvasive test such as used will in years to come provide more valuable information about symptomatic and asymptomatic recurrent stenosis after carotid endarterectomy. 


\title{
Chapter 7
}

\author{
General Discussion.
}

Stroke ranks as the second most common cause of death due to vascular disease in the United States ${ }^{108}$. In addition, it is also the cause of disability for an estimated one million Americans. Episodes of transient ischemic attacks are recognized as precursors of stroke since approximately $40 \%$ of affected patients are likely to have a cerebral infarction within the first five years after the initial event ${ }^{172}$. Furthermore, at least $75 \%$ of patients with completed strokes will give a history of previous transient ischemic attacks.

The relationship between extracranial carotid artery disease and stroke became fully realized with the development of cerebral arteriography. Arteriographic studies indicated that $74 \%$ of patients with ischemic stroke syndromes had at least one significant lesion in the extracranial vasculature ${ }^{7.5}$. In most patients with transient ischemic attacks, arteriography will demonstrate that the common site of narrowing is at the origin of the internal carotid artery. For many years, only high grade stenoses or occlusions were considered important in the pathogenesis of transient ischemic attacks (hemodynamic theory). At present, emboli from ulcerated plaques are considered to be the most common cause of transient cerebral ischemia (embolic theory) ${ }^{166}$. It is now accepted that the release of trombi into the bloodstream from an ulcerated area within a plaque can lead to both transient and permanent neurological changes. While embolization is often difficult to prove, the best evidence for such occurrences is found in patients with transient monocular blindness where emboli may be seen in the retinal arteries ${ }^{120}$ "

The finding that the lesion responsible for emboli is generally localized at an accessible side, i.e. the carotid bulb, made surgical treatment possible. Good long term results after carotid surgery made carotid endarterectomy one of the accepted forms of treatment for patients with transient ischemic attacks ${ }^{16,9}$. Contrast arteriography remains the ultimate diagnostic procedure for patients, who are candidates for carotid surgery. Since contrast arteriography has a small but real risk of serious complications ${ }^{16,4}$, ideally only those patients considered candidates for carotid surgery should undergo this invasive procedure. This creates a need for accurate noninvasive tests which could be used to screen patients with suspected extracranial arterial disease.

Noninvasive methods are readily separated in indirect and direct tests. Indirect tests such as supraorbital Doppler or oculoplethysmography are the most common noninvasive tests employed today for the detection of extracranial carotid artery disease. The reason for this is to be found in the simplicity and low cost of these tests. Supraorbital Doppler studies alone are unsuitable as diagnostic test for 
patients with suspected extractanial carotid artery disease ${ }^{15}$. Pressure oculoplethysmography seems to be accurate for high grade stenoses. Eikelboom ${ }^{56}$ described the results, using this device, in 309 patients where the extent of carotid disease was documented by contrast arteriography. For stenoses of $65 \%$ or more, the sensitivity and specificity were $87 \%$ and $84 \%$ respectively.

There are two major disadvantages with all indirect tests: (1) minimal disease (non flow-pressure reducing lesions) cannot be detected. This is important when it is realized that $50 \%$ of the patients in Thiele's study 160 with TIA's did not have stenoses of sufficient magnitude to reduce pressure or flow; and (2) it is not possible to differentiate between total occlusions and high grade stenoses. This distinction is an important one from a diagnostic standpoint.

Direct noninvasive tests operate without or with imaging systems. Those operating without an imaging system are in general based upon the evaluation of welocity changes using continuous wave Doppler devices ${ }^{2,134,148}$. Direct noninvasive tests that employ imaging systems can be divided in those that provide a flow map and those which provide a $\mathbf{B}$-mode image.

The continuous wave ultrasonic arteriograph, as described by Spencer et al ${ }^{153}$ provides a flow imaging system, which is accurate in predicting high grade stenoses of the carotid system. However, Weaver et al. ${ }^{177}$ found that lesions in the $0-50 \%$ diameter reduction range were not readily detected using this device. This is not surprising, since differentiation between the presence or absence of spectral broadening, as a reflection of flow disturbance produced by minimal lesions, is on theoretical grounds impossible using a continuous wave device.

With the development of pulsed Doppler ultrasound it became possible to detect blood flow at discrete points across the arterial lumen ${ }^{7}$, and assess the velocity patterns proximal to, in and distal to sites of stenoses. To increase the clinical utility of the pulsed Doppler, it is necessary to combine it with a method of acoustical imaging. There are a variety of approaches to this problem. Hokanson ot al. ${ }^{* 7}$ were the first to develop an ultrasonic arteriograph, which provides anatomical information by generating a flow map that can be used as a guide for precise placement of the pulsed Doppler sample volume.

Clinical results from the University of Washington, using Hokanson's ultrasonic arteriograph, were reported by Blackshear et al. ${ }^{24}$. The agreement between this ultratsonic device and contrast arteriography was $91 \%(20 / 22)$ for $50-99 \%$ diameter reductions, and $59 \%(13 / 22)$ for $10-49 \%$ diameter reductions. There was one false negative in the 8 normal vessels included in the study. A larger series, using Hokanson's arteriograph, was reported by Sumner et al. ${ }^{163}$. Using a diameter reduction greater than $20 \%$ as criterion for a positive test, the sensitivity of the method was $81 \%$ and the specificity $88 \%$ in 209 carotid bifurcations. These results demonstrate that ultrasonic pulsed Doppler imaging systems can be used to detect extracranial carotid artery disease encompassing the spectrum of involvement from normal to total occlusion. The system is relatively inexpensive and when combined with spectral analysis is suitable for both clinical and research orientated studies. 
The initial attempts to evaluate carotid disease with a B-mode system alone quickly demonstrated its deficiences. The first patient studied with this device at the University of Washington appeared to have a patent intermal artery on the B. mode image, but was found to have a total occlusion by contrast arteriography. Subsequent studies showed that both thrombus and non-calcified plaques may have the same acoustic properties as flowing blood ${ }^{76}$. This finding prompted the development of a device which combined the B-mode image system with a pulsed Doppler device. The B-mode image pernitted identification of the walls of the artery, visualization of calcified plaques, and served as a guide for the accurate placement of the sample volume of the pulsed Doppler at any desired point within the vessel. This concept of combining the two systems into the Duplex scanner was first described by Barber et al ". Since its initial development the system has undergone continuous modification to make it usable in clinical practice. This sequence and its results were described by Phillips et al. ${ }^{133}$.

For all of the validation studies, contrast arteriography has been used for comparison, since it is considered to be the standard for quantifying the location and degree of narrowing of the extracranial carotid arteries. Although considered the gold standard, it became apparent, that there was considerable variability in reporting the results of carotid arteriograms related to both the intra- and interobserver variability. Chikos et al. ${ }^{41}$ highlighted this problem in a carefully designed study of 128 multiplanar selective arteriograms of the carotid arteries. The intraobserver agreement for a "normal" internal carotid artery was $75 \%$ with an interobserver agreement of $57 \%$. This variability is one of the reasons that total agreement between arteriography and any noninvasive test, especially for evaluating normal and minimally diseased vessels, will probably never be possible.

A great deal of effort has been expended in attempting to assess the amount of diameter reduction of the internal carotid artery using spectral patterns obtained with the pulsed Doppler. Giddens et al. ${ }^{71}$ demonstrated, using hot film anemometry in an animal model, that flow disturbances can be devected immediately downstream of stenoses which do not reduce pressure or llow. Thiele et al. ${ }^{165}$ were able to verify this in a similar animal model using a high frequency pulsed Doppler velocimeter. These authors demonstrated that the pulsed Doppler could be used to assess the velocity disturbances that occur with varying degrees of stenoses. The most useful parameter to detect these flow disturbances was the estimation of spectral width. Hutchinson et al. " noted that broadening of the spectrum with non-pressure reducing stenoses occurred first during the deceleration phase of systole. As the stenoses became more severe (16-49\%), broadening was noted throughout the entire pulse cycle. In clinical studies of the carotid bifurcation, it is possible by combining the onset of spectral broadening with the peak systolic frequencies to estimate the degree of narrowing for discrete categories ranging from normal to total occlusion. Fell et al, have shown that using this approach it is possible to estimate the diameter reduction of the internal carotid artery with reasonable accuracy.

In January, 1980, the prototype PV III Duplex scanner was replaced by a commercially available unit. The improvements made were results of the previous: 
experience oblained with the earlier systems. The agreement between arteriography and ultrasonic Duplex scanning using the short focus scanhead for normal carotid arteries was $50 \%$, for the $5-15 \%$ diameter reduction category the agreement was $69 \%$ and for the $16-49 \%$ diameter reduction category $70 \%$. Using the medium focus scanhead agreement between arteriography and Duplex scanning was $94 \%$ for the $50-99 \%$ diameter reduction category and $93 \%$ for total occlusions.

The accuracy in differentiating between high grade stenoses and total occlusions improved as compared with previous results of the PV III Duplex scanner ${ }^{59}$. This was a result of the appreciation of the changes in the velocity patterns of the common carotid artery (see 5.2.). The capability to differentiate accurately between high grade stenoses and total occlusions makes the Duplex method superior to all indirect noninvasive tests.

The introduction of an algorithm to estimate the degree of involwement in patients with stenoses in the $50-99 \%$ diameter reduction category is promising and will likely play a role in documenting progression of disease for laesions in this category ${ }^{105}$.

Analyzing the results of the Duplex Scanner combined with spectral analysis and comparing them with other noninvasive methods, we are confident that this system provides an accurate screening method for patients with suspected extracranial carotid disease ${ }^{37}$. The method is suitable for screening patients who are considered possible candidates for contrast arteriography. Furthermore, since the method has the potential to detect disease varying from minimal lesions to total occlusions, the method may be suitable for studies designed to document the progression of disease.

Given the accuracy of Duplex scanning, will it replace contrast arteriography? At present the answer must be no, but it will clearly modify the indications for the invasive study. Since the technique is relatively new, each laboratory which employs the method will have to be confident of their results before basing clinical decisions on the findings of the Duplex study. In our laboratory, the accuracy of the method for the detection of high grade lesions and total occlusions approaches 94\%. Thus, in certain patients, it is entirely reasonable to base a decision to proceed with arteriography on the results of a Duplex scan.

Another question frequently asked is the role of scanning in patients with a clinical presentation that would normally demand arteriography. Should these patients be subjected to Duplex scanning or is it superfluous? Our feeling is that they should be studied for the following reasons: (1) there are situations in which the Duplex studies may be instrumental in the diagnostic approach that is followed. For example, the finding of a totally occluded internal carotid artery may remove the need for arteriography; (2) the noninvasive studies will provide a baseline examination that will be useful for follow-up; and (3) prospective studies of patients with extracranial arterial disease will require repetitive follow-up of such patients.

Of the patients referred for study, about one-third have focal TIA's as the basis for the referral (see 6.2.). Short of those found to have a total occlusion, there is 
general agreement that arteriography should be done to determine the basis for the problem. However, the decisions with the remaining wo-third of patients who do not have focal TIA's, the problem is more complex. For example, the group of patients with nonfocal symptoms present difficult problems, especially when the clinical picture is vague which is often the case. We are more willing to perform arteriography in this group of patients when bilateral disease is found by the Duplex study, especially when a total occlusion is found in one internal carotid artery with a high grade stenosis on the other side. The literature for this group of patients is rather confusing, although most authors consider bilateral high grade stenoses (diameter reduction of more than $50 \%$ ) a cause for the nonfocal symptoms $62,65,119$.

Our own approach to patients with asymptomatic bruits has been largely conservative both from the standpoint of arteriography and surgery. One of the reasons for this attitude is that it is still not clear that carotid endarterectomy will protect these patients for stroke. Recent publications $82,181,182$ have demonstrated that, although the overall risk for stroke is increased in this group of patients, the strokes that do occur may not be related to the side of the bruit. Thus, an asymptomatic bruit appears to be a good predictor of subsequent cardiovascular events, i.e. myocardial infarction and stroke. Since there is a need for prospective studies to obtain information about the natural history of this group of patients, we have embarked upon such a study. It will require another five years before the results of this study will provide meaningful data.

Given our own policy, it was of interest to see what referring physicians did with the information provided to them from our laboratory (see 6.2.). A surprising finding was that even though patients were classified as having focal symptoms lasting less than 24 hours by the referring physicians, there remained a considerable number who did not have arteriography. Only $22 \%$ in the $5-15 \%$ diameter reduction group and $27 \%$ in the $16-49 \%$ diameter reduction group underwent contrast arteriography. Whether this approach is a correct one can only be answered by follow-up studies. It is our plan lo follow the patients who did not undergo arteriography because it is only by this approach that we can find the answer to this question. It also became apparent that the referring physicians considered the finding of a high grade stenoses of greater clinical importance in both the nonfocal and asymptomatic bruit group (see 6.2. and 6.3.). The number of patients with a $50-99 \%$ diameter reduction who underwent arteriography in these two groups was significantly higher than those with lesser degrees of involvement.

Whether or not prophylactic carotid surgery should be performed in patienls who are candidates for coronary bypass surgery is still controversial. As a result of our study on 102 patients (see 6.4.) and Barnes studies ${ }^{14 .}{ }^{16}$, we no longer advocate arteriography prior to coronary by pass surgery even if high grade stenoses are found in patients without a history of focal neurological symptoms. This does not mean that the patients with suspected carotid disease should not be screened. It would appear reasonable to counsel the patient and referring physician that disease is present and continued regular follow-up is to be carried out until the natural history of asymptomatic high grade lesions is known. 
Although the indications for carotid surgery are not well established to everyone's satisfaction, carotic endarterectomy is the most commonly performed vascular procedure in the United States of America. In good centers, the procedure can be performed safely with a low complication rate. This fact does not appear to be widely accepted by some neurologists as evidenced by the differing views concerning the role of endarterectomy. There is no doubt that the results do vary from region to region and reflect the skill and experience of the surgeon. Whenever carotid endarterectomy is indicated, it should only be done by surgeons whose complication rate is less than $2 \% 171$.

Although carotid endarterectomy appears to be a durable procedure with good. results, little is known about the hemodynamic changes that follow the operation. Our results from 88 patients with a follow-up of two years demonstrate that recurrent asymptomatic restenoses is found in $9 \%$ after three months, $18 \%$ after six months, $19 \%$ after twelve months, and $17 \%$ after twenty four months followup. Longer follow-up studies will be required to assess the importance of restenosis with respect to the development of recurrent symptoms. It is our impression that the Duplex scanner is an ideal means of following patients postoperatively. The method provides hemodynamic data on the flow patterns across the operated segment. The linding of flow disturbances is extremely common even when high grade stenoses are not found. If these flow disturbances are a factor in the cause of myointimal hyperplasia or secondary to such a change remains to be determined.

There have been reports in the literature describing the use of objective vellocity waveform parameters for the detection and classification of disease of the carotid arteries. Blackshear et al. ${ }^{27}$ and Knox et al. ${ }^{115}$ introduced simple parameters to quantitate the degree of stenosis of the internal carotid artery. Rutherford et al. ${ }^{148}$ used discriminant analysis of selected waveform parameters to identify normal and diseased internal carotid arteries. Greene et al. ${ }^{73}$ at the University of Washington developed a similar technique using computer selected waveform parameters employing a stepwise discriminant analysis to classify disease into four categories. Data from the Duplex scanner with ECG data are recorded on audiotape for further analysis. The Doppler information of 20 suitable heart beats are chosen by the computer and subjected to the fast Fourier spectrum analysis from which an ensemble averaged spectrum is produced. The averaged spectrum is then analysed by a software package developed to perform pattern recognition 73. A stepwise algorithm is used to go through the following decisions: (1) normal or diseased; (2) if diseased, greater or less than $50 \%$ diameter reduction; and (3) if less than $50 \%$, greater or less than $20 \%$ diameter reduction.

Al present, 24 normal internal carotid arteries and 81 diseased internal carotid arteries with arteriographic confirmation have been analysed. Of the 24 normal internal carotid arteries 18 were so classified by the computer. Of the 10 internal carotid arteries with $5-15 \%$ diameter reduction, 7 were classified correctly by the computer. Of the 39 with a $16-49 \%$ diameter reduction, 19 were classified correctly, and of the 32 with $50-99 \%$ diameter reduction, 22 were correctly identified by the computer. These results are promising, although more work has 
to be done before a microprocessor can be developed which can be used on-line to make these decision steps.

What are the research goals for the future? Basically these can be divided into two categories: (1) signal processing and (2) long-term clinical studies.

Signal processing will follow two directions. The computer recognition programs will have to be relined further with the goal of developing a microprocessor which can be used on-line to analyze the Doppler spectrum at the time the patient is being studied. In addition, work is ongoing to evaluate the feasibility of analyzing averaged data which varies from 1-20 heart cycles. Dr. D.J. Phillips has started on this latter program at the University of Wastington using data from the Duplex scanner. This work is a logical, intermediate step between the current approach described in chapter five of this thesis and the more complex work, being done by F.M. Greene with the computer pattern recognition program ${ }^{73}$. One of the questions which needs to be answered is the number of cycles one should average to obtain a velocity waveform that is adequate for further analysis. Selecting too many cycles could result in the loss of non- periodic information through averaging that could be useful. Averaging too few can result in the analysis of nonmeaningful beat to beat variations. Having the option to change the number of heart cycles to be averaged, creates a new dimension for the analysis of spectral data. It is possible that some important parameters will be best depicted in an averaged velocity waveform where others may be observed only in a non-averaged waveform.

Documenting progression of disease will be the other main goal for future work. As has been stated previously, the Duplex concept is suitable for this sort of study because the method can be used repetitively to provide accurate reproducible hemodynamic information concerning the carotid bifurcation. Based upon our experience, we are confident that this important issue can best be addressed by obtaining repetitive velocity data from the same anatomic sites.

However, which patient groups should be followed and which parameters should be chosen? The first part of this question is not difficult to answer. Basically, those patients should be followed in which progression of disease is of clinical importance. It is clear that there is a need for information about the natural history of patients with asymptomatic bruits. The next group of patients are those who have undergone carotid surgery. Does restenosis play a role in the development of recurrent symptoms? Another group of patients, where the longterm follow-up is important are those patients with TIA's not treated surgically. Perhaps, it will be possible to answer the question concerning the role of antiplatelet agents in preventing disease progression.

At present it is not possible to decide which parameters should be chosen to document progression of disease. The computer pattern recognition program as it stands today does not seem to be sufficient, since diameter reduction changes within the 50-99\% category cannot be documented. This will require the addition of new algorithms such as described by Knox et al. ${ }^{105}$ to cover the entire spectrum of disease. Although many problems remain to be solved, it seems clear that using the averaged velocity waveforms from the common and internal carotid artery 
with the help of computer controlled stepwise discriminant analysis will be sufficient to answer these questions.

In conclusion, the results of the studies presented in this thesis have answered only a few of the questions concerning the diagnosis and treatment of extracranial carotid artery disease. With such a method available to clocument the extent of disease, the ultimate question comes to mind: Is it possible to predict clinical outcome on the basis of the degree of diameter reduction of the internal carotid artery? This question has not yet been answered and it will take probably another ten years before the answer is found. However, it can be expected that noninvasive tests such as described will be useful in approaching this problem objectively. A great deal of knowledge about this difficult issue is being slowly accumulated by people working in the field of ultrasound. As long as the noninvasive studies are used to carry out long-term studies, progress with regard to the natural history of carotid atherosclerosis will be made. 


\section{Summary}

The studies in this thesis evaluate the practical value of ultrasonic Duplex scanning combined with spectral analysis in the detection of extracranial carotid artery disease. The method is based on the combination of real-time B-mode imaging and a $5 \mathrm{MHz}$ pulsed Doppler device. The B-mode images are used as a guide for placement of the sample volume of the pulsed Doppler in the centerstream of the vessel of interest. The basic principle of the method is the estimation of diameter reductions of the internal carotid artery with velocity waveform parameters obtained from the pulsed Doppler device, subjected to Fast Fourier Transform spectral analysis.

In four studies the issue of the accuracy of the ultrasonic method, as compared to multiplanar contrast arteriography, were presented. The first study evaluated the value of velocity waveform information of the common carotid artery in predicting disease in the internal carotid artery. In the second study the accuracy of Duplex scanning was evaluated in 246 carotid bifurcations. The third study concerned the evaluation of velocity waveform changes due to an increase of total blood flow in normal subjects as a means to unmask lesser degrees of stenoses. The fourth study concerned the evaluation of a parameter for accurate detection of severe carotid artery disease.

Given a certain accuracy the ultrasonic device was used in four clinical orientated studies. The first two studies concerned the influence of Duplex scanning on the managment of referring physicians in patients with symptomatic and asymptomatic extracranial carotid artery disease. The third study evaluated the incidence of carotid artery disease in patients who were candidates for coronary bypass surgery, the fourth study concerned the issue of recurrent stenoses after carotid surgery.

Chapter 1. This chapter provides the reader with a short review of the anatomy and pathology of the extracranial carotid artery systems. Clinical considerations of carotid artery disease are discussed.

Chapter 2. Contrast arteriography is traditionally considered the screenings method of carotid artery disease. The use of this invasive procedure both as screenings device and gold standard for noninvasive methods is described. The variability in reading arteriograms, especially with no or minimal disease present, and the consequences of this variability for noninvasive methods are mentioned.

The second part of this chapter is a review of the noninvasive screenings techniques available for detecting extracranial carotid artery disease. Basicly they can be divided in indirect and direct noninvasive tests. The method used in this thesis is a direct ultrasonic test, obtaining information from the carotid bifurcation

Chapter 3. In this chapter some basic principles of fluid dynamics are introduced. Changes in the velocity profiles of the carotid system obtained by the Doppler technique will be more meaningful with the knowlegde of these basic principles. 
Chapter 4. This chapter describes the current status of ultrasonic techniques. The fundamental differences between continuous wave and pulsed Doppler are described. Detecting velocity information from discrete points in the vessel of interest, as provided by a pulsed Doppler system, justifies the use of a parameter like spectral broadening. This parameter allows the detection of arteriographic lesions which are non pressure or flow reducing. The additional anatomical information, required to obtain relevant pulsed Doppler information, is with Duplex scanning provided by B-mode images.

Using five categories, the pulsed Doppler information combined with spectral analysis can be classified as followed:

(A) normal, 0\% diameter reduction; (B) 5-15\% diameter reduction; (C) 16-49\% diameter reduction; (D) 50-99\% diameter reduction; and (E) total occlusion, $100 \%$ diameter reduction. The parameters used for this classification are: (1) spectral width during systole; (2) systolic peak frequency; and (3) diastolic frequencies.

Chapter 5 . The basis for this chapter are four studies performed with both data from the prototype PV III Duplex scanner and the commercially available Mark V Duplex scanner. The first study was performed to assess in an objective fashion the frequency of flow to zero during late systole or diastole in the velocity waveform of the common carotid artery as related to disease in the proximal internal carotid artery. Of 156 patients, who underwent multiplanar contrast arteriography, the velocity patterns of the common carotid artery were reviewed. Flow to zero during diastole in the common carotid artery was found in three groups of patients: (1) patients with flow to zero in both common carotid arteries not related to disease in the proximal internal carotid artery; (2) patients with a tight stenosis (90-99\% diameter reduction) in the internal carotid artery; and (3) patients with a total occlusion of the internal carotid artery. Although flow to zero during diastole in the common carotid artery is very commonly observed with a total occlusion of the internal carotid artery, it may also be seen with a high grade stenosis. Thus when lound, it is essential to ascertain if flow is, indeed, present in the internal carotid artery. The appreciation of the characteristics of the velocity pattern of the common carotid artery improved the overall accuracy in detecting extracranial carotid artery disease.

The second study presented the results concerning the accuracy of the Mark $\mathrm{V}$ Duplex scanner combined with spectral analysis. For comparison between multiplanar contrast arteriography and the ultrasonic device were available 246 carotid sides, 169 sides were studied with the medium and 77 sides with the short focus scanhead. The agreement between arteriography and ultrasonic Duplex Scanning using the short focus scanhead for normal carotid arteries was $50 \%$, for the $5.15 \%$ diameter reduction category $69 \%$, and for the $16-49 \%$ diameter reduction category $70 \%$. These results were clearly better than the results obtained with the medium focus scanhead in the same three categories.

The agreement between arteriography and ultrasonic Duplex scanning in high grade stenoses (50-99\% diameter reduction) was $94 \%$, using the medium focus scanhead and $89 \%$ using the short focus scanhead. The agreement for total 
acclusions was $93 \%$ for the medium focus scanhead and $100 \%$ for the short focus scanhead. In high grade lesions and total occlusions there was no marked difference between the two scanhead.

The third study was designed as pilot study in order to find a means of unmask lesser degrees of stenoses and separate them from normal carotid arteries. Five normal subjects increased the total blood flow to their brains by breathing $6.8 \%$ carbon dioxide. Changes in flow were quantified by features derived from the velocity waveforms of the common, internal, and external carotid artery. An increase in velocities during diastole correlated well with the expected increase in total blood flow induced by breathing $6.8 \%$ cambon dioxide. Increases in spectral width reflected the development of flow disturbances not apparent under basal conditions. The study suggests that marked increase in flow produced by intracranial vasodilatation can dramatically alter the velocity patterns normally observed.

The fourth study concerned the evaluation of an algorithm for accurate detection of severe carotid artery disease. The study was performed in order to estimate the degree of involvement in the 50-99\% diameter reduction category. The algorithm used is the ratio of peak systolic velocity in the internal carotid artery to the end diastolic velocity in the common carotid artery. The ratio was evaluated in 68 diseased and 30 normal carotid arteries. The algorithm permitted gradation of disease into $60 \%$ diameter reduction and more with $95 \%$ accuracy, $65 \%$ diameter reduction and more with $97 \%$ accuracy, and $90 \%$ diameter reduction and more with $100 \%$ accuracy. The values of the 30 normal sides studied, fell below those of the $60 \%$ diameter reduction group. The algorithm seems to be useful in further gradation of stenoses in the $50-99 \%$ diameter reduction range.

The four studies of this chapter demonstrated that the accuracy of ultrasonic Duplex scanning combined with spectral analysis justifies the use of the method as a screenings device for patients with extracranial carotid artery disease.

Furthermore, the method has the potential to document progression of disease. Critical, however, is the knowlegde of the limitations of the method, as has been shown by the use of the medium focus scanhead in patients with minimal disease.

Chapter 6. This chapter is based on four clinical studies performed with the Mark V Duplex scanner combined with spectral analysis. The first study was performed to assess the influence of the Duplex studies on the managment of patients with suspected symptomatic extracranial carotid artery disease. Data on all new referrals $(n=410)$ during one year were reviewed. The patients were grouped according to their reason of referral: (1) patients with focal neurological symptoms lasting less than 24 hours $(n=156)$; (2) patients with focal symptoms lasting longer than 24 hours $(n=107)$; and ( 3 ) patients with nonfocal neurological symptoms $(n=147)$. For all groups there was a statistically significant relationship between the reporting of the presence of a high grade stenosis $(50-99 \%$ diameter reduction) and the likelihood that the patient would undergo contrast arteriography. The finding of a high grade stenosis on the arteriograms resulted in a greater number of patients undergoing carotid surgery. The results of this study indicate that the report of the noninvasive test influenced the decision making process in the 
management of patients with suspected carotid artery disease. For patients with focal neurological symptoms lasting less than 24 hours this was a surprising finding, since it is our own policy that these patients will undergo contrast arteriography regardless the percentage of stenosis.

In the second study 95 patients referred to the vascular laboratory because of an asymptomatic bruit were evaluated with the Mark V Duplex scanner. Unilateral bruits were found in 48 patients and bilateral bruits in 47 patients. Out of the 142 carotid sides with a bruit $2 \%(3 / 142)$ were found to be normal, 30\% (42/142) were found to have a $5-15 \%$ diameter reduction, $37 \%(53 / 142)$ were found to have a $16-$ $49 \%$ diameter reduction, $29 \%(42 / 142)$ had a $50-99 \%$ diameter reduction, and $2 \%$ (3/142) of the patients were found to have a total occlusion of the internal carotid artery. When a high grade stenosis (50-99\% diameter reduction) is considered to be an

indication for contrast arteriography then this invasive procedure could be avoided in $71 \%$ of the cases. Our own approach of this problem is largely conservative both from the standpoint of arteriography and surgery. Even in patients who are candidates for coronary bypass surgery there is evidence that profylactic carotid surgery is not indicated for patients without neurological symptoms. We perform Duplex studies in patients with asymptomatic bruits as a part of a follow-up study in order to address the issue of documenting progression of disease. Only such studies will give the answer whether or not profylactic surgery should be performed in patients with asymptomatic carotid disease such as asymptomatic bruits.

The third study was performed to obtain a better understanding of the incidence of carotid occlusive disease in candidates for coronary bypass surgery and evaluate the practical use of a noninvasive method to screen patients preoperatively. Hunderd and two patients were evaluated preoperatively with the Mark V Duplex scanner, 24 studies were requested on the basis of either previous neurological problems or the presence of a bruit and 78 studies were performed as part of the siudy protocol. In the unrequested group $6 \%(5 / 78)$ of the patients had a stenosis of $50 \%$ dianeter reduction or more. In the requested group $54 \%$ (13/54) had a stenosis of $50 \%$ diameter reduction or more. In the postoperative phase one stroke and one transient ischemic at tack were noted among the unrequested group and none in those suspected of having carotid disease on clinical grounds. Neither patient had a high grade stenosis. Based on this study and others there is tittle evidence to support the contention that noninvasive screening of patients free of symptoms or signs of carotid disease prior to coronary artery bypass surgery is a valuable method of screening patients who will sustain a focal neurological event.

In the fourth study sequential changes in the velocity patterns after carotid endarterectomy were evaluated, 88 patients were studied prior to and following carotid surgery with the ultrasonic Duplex device. Patients were studied at three, six, twelve and twenty-four months after surgery. The velocity patterns of all patients demonstrated flow disturbances following carotid endarterectomy. Velocity patterns consistent with recurrent high grade stenoses (more than $50 \%$ diameter reduction) were found in $9 \%$ at three months, $18 \%$ at six months, $19 \%$ at twelve months, and $17 \%$ at twenty-four month follow-up. Most restenoses are 
considered to be caused by myointimal hyperplasia, which could be the result of the documented flow disturbances. The results of this study demonstrated that: (1) flow disturbances occur after carotid endarterectomy even without recurrent stenoses; and (2) the development of early restenoses is higher than the incidence of recurrent symptoms.

Chapter 7. In the general discussion the most prominent findings of chapter 5 and 6 are reviewed. These findings demonstrate that Duplex scanning combined with spectral analysis is an accurate method to detect extracranial carotid artery disease. The method has proven to be suitable for clinical and research orientated study protocols.

Further goals for the research orientated studies will be: (1) to improve signal processing, and (2) to find algorithms to document progression of extracranial carotid artery disease. These algorithms will probably be found with the development of a variable averaging spectrum velocity pattern program combined with a computer controlled stepwise discriminant analysis program. 


\section{Samenvatting}

Gedurende de laatste jaren heeft het "niet-invasieve" onderzoek van de extracraniële cerebrale circulatie een stormachtige ontwikkeling doorgemaakt. Deze ontwikkeling is toe te schrijven aan een toenemende behoefte op gefundeerde gronden patienten te kunnen selecteren voor contrastarteriografie. Dit proefschrift beschrijft een niet-invasieve methode met als doelstelling de klinische betekenis ervan te evalueren. De gebruikte methode is gebaseerd op het "Duplex" principe, een combinatie van echografie en een pulsed-Doppler systeem. De met echografie verkregen anatomische informatie stelt het pulsed-Doppler systeem in stat een representatief snelheidsprofiel van de extracraniële vaten te verkrijgen. Spectraal-analyse van het snelheidsprofiel maakt het vervolgens mogelijk, aan de hand van hemodynamische parameters, de diameterreductie van een arterie te bepalen.

Traditioneel wordt de nauwkeurigheid van niet-invasief onderzoek bepald door vergelijking met contrast-arteriografie, waarbij de arteriografie als "gouden standaard" wordt gebruikt. In dit proefschrift wordt de nauwkeurigheid van het Duplex principe in combinatie met spectraal- analyse atan de hand van de resultaten van vier studies besproken.

Naast de functie om patiënten te kunnen selecteren voor arteriografie kunnen met een nauwheurig niet-invasief onderzoek carotis afwijkingen in bepaalde patiënten populaties worden geidentificeerd en eventueel vervolgd. Het doel van deze studies, waarvan er vier in dit proefschrift zijn opgenomen, is het verkrijgen van meer inzicht in de betekenis van de aanwezigheid van arteriosclerotische carotis afwijkingen en het optreden van eventuele progressie.

In hoofdstuk 1 wordi een overzicht gegeven van de anatomie en pathologie van de extracraniële cerebrale circulatie. De klinische betekenis en eventuele behandeling van arteriosclerotische afwijkingen ter hoogte van de carotisbifurcatie worden besproken.

Hoofdstuk 2. Diagnostisch onderzoek van de carotis-bifurcatie bestatal uit invalsief en niet-invasief onderzoek. Contrast-arteriografie wordt traditionecl beschouwd als het definitieve onderzoek om carotis-bifurcatie alwijkingen te diagnostiseren. De betrouwbararheid en risico's van dit invasieve onderzoek worden besproken.

Niet-invasief onderzoek kan worden ingedeeld in "indirecte" en "directe" methoden. Indirecte methoden registre ren veranderingen in het collateralle systeem ten gevolge van carotis-bifurcatie afwijkingen, directe methoden registreren ter plaatse van de bifurcatie. Het Duplex concept is gebaseerd op registraties ter hoogte van de carotis-bifurcatie en is daardoor een directe methode.

Hoofdstuk 3. Met behulp van hemodynamische parameters wordt het geanalyseerde snellheidsprofiel geclassificeerd; de parameters zijn spectrale verbreding, maximale systolische en diastolische frequenties. Met bekendheid van enkele hemodynamische basisprincipes krijgt het gebruik van de gekozen parameters meer betekenis. Relevante principes worden in het kort beschreven. 
In hoofdstuk 4 worden de kemmerken en verschillen van continuous wave en pulsed-Doppler technieken beschreven. Een pulsed-Doppler systeem stelt de gebruker in stat hemodynamische informatic wanuit het midden van een arterie te verkrijgen. Hierdoor is het mogelijk veranderingen in de spectrale verbreding wan het snelheidsprofiel, als gevolg van minimale arterioscle rotische watwandalwikingen, te registreren. In het Duplex concept wordt de anatomische informatic, nodig len einde de localisatie van het pulsed-Doppler systeem te bepalen, verkregen door echografie.

Gebruik makend van eerder genoemde parameters kan het snelheidsprofiel van de arteria carotis interna in één van de volgende vijf categoriën worden ingedecld: (A) normal, $0 \%$ diameterreductie; (B) 5-15\% diameterreductic; (C) 16-49\% diameterreductie; (D) $50-99 \%$ diameterreductie; on (E) totale occlusie, $100 \%$ diameterreductie.

In hoofdstuk 5 wordt aan de hand van de resulkaten van vier studies de nauwkeurigheid van het Duplex concept in combinatie met spectral- analyse besproken. In de eerste studie wordt het snelheidsprofiel van de arteria carotis communis gebruikt om de nauwkeurigheid van het registreren van arteria interna afwijkingen te verhogen. Het normale arteria carotis communis profiel vertoont cen continue voorwartse stroomrichting gedurende de diastole fase. Deze continue voorwatstse stroomrichting blijkt in drie situaties niet aanwezig te zijn: (1) bij patiënten met een totale occlusie van de arteria carotis interna; (2) bij patienten met een $90-99 \%$ diameterreductie van de arteria carotis interna; en (3) bij patienten met centrale pathologie, niet gerelateerd a nateria carotis interna afwijkingen. In deze latste categorie is de voorwautse stroom richting beiderzijds niet alanwezig. Met de waardering van de veranderingen in hot snetheidsprofiel wan de arteria carotis communis, is de nauwkeurigheid van het diagnostiseren van totale occlusies toegenomen.

De weede studie geeft aan de hand van een vergelijkende studie de natuwkeurigheid van het Duplex concept in combinatie met spectral-analyse. Gobruik makend van cen "short locus" transducer is de overeenstemming tussen Duplex en arteriogralie $50 \%$ voor cattegorie A (0\% diameter reductie), $69 \%$ voor calcgorie B (5-15\% diameterreductic), en $70 \%$ voor categorie $\mathrm{C}(16-49 \%$ diameterreduclie). Gebruik makend van een "medium focus" transducer is de overeenstemming ussen Duplex en arteriografie $94 \%$ voor categorie D $(50-99 \%$ diameterreductio) en $93 \%$ voor categorie E (totale occlusic).

De resultaten van dit vergelijkende onderzoek tonen aan dat het niet-invasieve onderzoek 50-99\% diameterreducties en totale occlusies met een hoge naluweurigheid registreent. Het scheidend vermogen tussen een normale arteria calrotis interna en een $5-15 \%$ diameterreductie zou theoretisch kumen worden vergroot door de totale hoeveelheid bloed per tijdseenheid dramatisch te verhogen. Teneinde het snelheidsprofiel van een normal carotis systeem te kunnen beoondelen bij een toename van de totale bloedstroom werd in de derde studie bij vijl gezonde proelpersonen voor en tijdens $\mathrm{CO}_{2}$-inhalatie een Duplex studie verricht. Ten gevolge van de $\mathrm{CO}_{2}$-inhalatie trad bij alle proefpersonen. als uiting van een vermindering van de stabiliteit van de bloedstroom, een verbreding van het spectrum in het snelheidsprofiel op. Alvorens de warde van dit fenomeen 
te kunnen beoordelen, zal de studie eveneens verricht moeten worden met patiênten bekend met een $5-15 \%$ diameterreductie van de arteria carotis interna.

In de vierde studie wordt een nieuwe parameter geintroduceerd, met behulp warvan afwijkingen in de $50-99 \%$ diameterreductie categorie kunnen worden herverdeeld. Met deze parameter blijkt het mogelijk een $90-99 \%$ diameterreductie categorie te creëren, wardoor toename van arteriosclerotische afwijkingen in follow-up studies voor de $50.99 \%$ diameterreductie categoric kunnen worden gedocumenteerd.

Hoofdstuk 6 bestaat wit vier klinische studies die de invloed en betekenis van het gebruik van het Duplex principe in patiënten populaties evalueren.

In de eerste studie wordt de invloed van het niet-invasieve onderzoek op de verdere behandeling van patiënten met neurologische symptomen besproken. Het vermelden van een $50-99 \%$ diameterreductie van de arteria carotis interna blijkt. onafhankelijk van het klachtenpatroon, de kans op arteriografisch onderzoek significant te verhogen.

De tweede studie registreert het voorkomen van afwijkingen in de arteria callot is interna bij patiënten met een asymptomatische souffle in cle hals. Van de 142 carotis-bifurcaties met een souffe in de hals wordt de arteriat carotis interna in $2 \%$ geclassificeerd als normal, in $30 \%$ met een $5-15 \%$ diametereductie, in $37 \%$ met een $16-49 \%$ diameterreductie, in $29 \%$ met een $50-99 \%$ diameterreductie en in $2 \%$ met een totale occlusie. Als een hemodynamisch significante stenose een indicatie is voor contrast-arteriografie in patiënten met een asymptomatische soufle, kan het niet-invasieve onderzoek dit invasieve onderzoek $71 \%$ van de patiënten besparen.

In de derde studie wordt het voorkomen van carotis afwijkingen, in patienten die coronair chirurgie ondergan, gedocumenteerd. In $6 \%$ van de patiënten zonder neurologische symptomen werd een $50-99 \%$ diameterreductie van de arteria carotis interna geregistreend. De optredende peri-operatieve neurologische complicaties waren niet gerelateerd aan het voorkomen wan hemodynamisch significante stenosen.

In de vierde studie worden hemodynamische veranderingen geregistreen in patiènten die carotis chirurgie hebben ondergaan. De resultaten van dere studic tonen aan dat een postoperatieve restenosering over het algemeen asymptomatisch verloopt en relatief frequent optreedt.

Hoofdstuk 7. In de algemene discussie worden de belangrijkste bevindingen van hoofdstuk 4 en 5 nader besproken. De resultaten van de eerste vier studies tonen atan, dat het niet-invasieve Duplex onderzoek in combinatie met spectraal-analyse nauwkeurig arteria carot is interna alwijkingen registrean. De methode is principieel superieur aan elke indirecte methode door de naw weurigheid warmee een hemodynamische significante stenose kan worden onderscheiden van een totale occlusie. De vier studies van hoofdstuk 6 demonstreren de klinische waarde van een nauwkeurig niet-invasief onderzoek voor de carotis-bifurcatic allwijkingen.

Toekomstig werk met de Duplex scanner dient te zijn gericht op het verbeteren 
van het scheidend vermogen ussen een normale arteria carotis interna en een arteria met minimale arteriosclerotische afwijkingen. Verder zullen een aantal follow-up studies worden verricht ten einde een beter inzicht te krijgen op het natuurlijke verloop van arteriosclerotische carotis-bifurcatiea fwijkingen. 


\section{References}

1. Archie, J.P.:

A simple, non-dimensional, normalized common carotid doppler velocity wave-form index that identified patients with carotid disease.

Stroke 12: 322, 1981.

2. Ashor, G.W.; Meyer, B.W.; Lindesmith, G.G.; Stiles, Q.R.; Walker, G.H.; Tucker, B.L.:

Coronary artery disease. Surgery in 100 patients 65 years of age and older. Archives of Surgery 107: 30, 1973.

3. A.T.L.

720 Ultrasonic Scan Head Operation Manual.

A.T.L. Laboratories, Inc., Bellevue, Wa, U.S.A..

4. Allen, N.; Mustain, V.:

Origin and significance of vascular murmurs of the head and neck.

Medicine $41: 227,1962$.

5. Attinger, E.O.; Sugawara, H.; Navarro, A.; Anne, A.:

Pulsatile flow patterns in distensible tubes.

Circulation Research 18:447, 1966.

6. Attinger, E.O.; Sugawara, H.; Navarro, A.; Ricetto, A.;

Martin, R.:

Pressure-flow relations in dog arteries.

Circulation Research 19: 230, 1966.

7. Baker, D.W.:

Pulsed ultrasonic Doppler blood-flow sensing.

IEEE Trans, on Sonics and Ultrasonics 17: 170, 1970.

8. Baker, D.W.; Johnston, S.L.; Strandness, D.E. Jr.."

Prospects for quantitation of transcutaneous pulsed Doppler

techniques in cardiology and peripheral vascular disease.

In: Cardiovascular Applications of Ultrasound.

Ed. R. Reneman. North-Holland. Publ. Co., 1974.

9. Baker, D.W.; Strandness, D.E. Jr.; Johnson, S.L.:

Pulsed Doppler techniques: some examples from the University of Washington.

Ultrasound in Med. and Biol. 2: 251, 1976.

10. Baker, J.D.; Barker, W.F.; Machleder, H.I.:

Ocular Pneumoplethysmography in the evaluation of carotid stenosis.

Circulation 62, supp. 1, 1980. 
11. Barber, F.E.; Baker D.W ; Nation A.W.C., Strandness, D.E. Jr ; Reid J.M.:

Ultrasonic duplex echo-Doppler scanner.

IEEE Trans, on Biomedical Engineering 21: 109, 1974.

12. Barber, F.E.; Baker, D.W.; Strandness, D.E. Jr.; Ofstad, J.M.; Mahler, A.D.* Duplex scanner 11 : for simultaneous imaging of artery tissues and flow.

Ultrasonic Symposium Proceedings, IEEE Cat. ... 74 CHO 896 ISU, 1974.

13. Barnes, R.W.; Russell, H.E.; Bone, A.E.; Slaymaker, E.E.:

Doppler cerebrovascular examination: improved results with refinements in technique.

Stroke 8: 468,1977 .

14. Barnes, R.W.; Marszalek, P.B.; Rittgers, S.E.:

Asymptomatic carotid disease in preoperative patients.

Stroke 11: 136, 1980.

15. Barnes, R.W.; Nix, L.; Rittgers, S.E.:

Audible Interpretation of Carotid Doppler Signals.

Archives of Surgery 116: 1185, 1981.

16. Barnes, R.W.:

Screening for asymptomatic carotid disease in patients undergoing cardiovascular surgery.

To be published in: Noninvasive Cardiovascular Diagnosis, Third Edition Ed.

Diethrich E.B.

\section{Barnett, H.J.M.:}

A randomized trial of aspirin and sulfinpyrazone in threated stroke.

N. Eng. J. Med. 299: 53, 1978.

18. Barnett, H.J.M.:

Progress toward stroke prevention: Robert Wartenberg Lecture.

Neutology 30: 1212, 1980.

19. Baskett, J.J.; Beasley, M.G.; Murphy, G.J.; Hyams, D.E.; Gosling, R.G.*

Screening for carotid junction disease by spectral analysis of Doppler signals.

Cardiovascular Research 11: 147, 1977.

20. Beach, K.:

Noninvasive Carotid Diagnosis: Entering the second Decade.

Bruit 4: 9, 1980.

21. Bergan, J.J.; Yao, S.T.:

Invited overview: Role of the vascular laboratory.

Surgery 88: 9, 1980 . 
22. Berguer, R.; Hwang, N.H.C.:

Critical arterial stenosis.

Annals of Surgery $180: 39,1974$.

23. Bernstein, E.F.; Dilley, R.B.; Goldberger, L.E.; Gasink, B.B.; Leopold, G.R.: Growth rates of small abdominal aortic aneurysms.

Surgery $80: 765,1976$.

24. Blackshear, W.M. Jr.; Philips, D.J.; Thiele, B.L.; Hirsch, J.H.; Chikos, P.M.; Marinelli, M.R.; Ward, K.J.; Strandness, D.E. Jr.:

Detection of carotid occlusive disease by ultrasonic imaging and pulsed Doppler spectrum analysis.

Surgery 86: 698, 1979.

25. Blackshear, W.M. Jr.; Thiele, B.L.; Harley, J.D.; Chikos, P.M.; Strandness, D.E. Jr.:

A prospective evaluation of oculoplethysmography and carotid phonoangiography.

Surgery, Gynecology and Obstretics 148: 201, 1979.

26. Blackshear, W.M.; Phillips, D.J.; Strandness, D.E. Jr.:

Pulsed Doppler assessment of normal human femoral artery velocity patterns. Journal of Surgicall Research 27: 73, 1979.

27. Blackshear, W.M.; Phillips, D.J.; Chikos, P.M.; Harley, J.P.; Thiele, B.L.; Strandness, D.E. Jr.:

Carotid artery velocity patterns in normal and stenotic vessels.

Stroke 11:67, 1980 .

28. Blackshear, W.M.; Phillips, D.J. ; Bodily, K.C.; Strandness, D.E. Jr.:

Ultrasonic demonstration of external and internal carotid patency with common carotid occlusion: A preliminary report.

Stroke 11:249, 1980 .

29. Blaisdell, F.W.; Clauss, R.H.; Galbraith, J.G.; Imparato, A.M.; Wylie, E.J.: Joint study of extracranial arterial occlusion. IV Surgical considerations. J.A.M.A. 209: 1889, 1969.

30. Bodily, K.C.; Zierler, R.E.; Marinelli, M.R.; Thiele, B.L.; Greene, F.M.; Sirandness, D.E. Jr.:

Flow disturbances following carotid endarterectomy.

Surgery, Gynecology and Obstretics 15: 77, 1980.

31. Bodily, K.C.; Phillips, D.J.; Thiele, B.L.; Strandness, D.E. Jr.: Noninvasive detection of internal carotid artery occlusion.

Angiology 32: 517, 1981 . 
32. Bone, G.E.; Barnes, R.W.:

Clinical implications of the Doppler cerebrovascular examination: A correlation with arteriography.

Stroke 7: 271, 1976.

33. Breslau, P.J.; Fell, G; Ivey, T.D.; Bailey, W.W.; Strandness, D.E. Ir.:

Carotid arterial disease in patients undergoing coronary artery bypass surgery. Journal of Thoracic and Cardiovascular Surgery $82: 765,1981$.

34. Breslau, P.J.; Knox, R; Fell, G.; Greene, F.M.; Thiele, B.L.; Strandness, D.E. Jr.:

Effect of carbon dioxide on flow patterns in normal extracranial arteries. Jourmal of Surgical Research, in press.

35. Breslau, P.J.; Fell, G.; Phillips, D.E.; Thiele, B.L.;

Strandness, D.E. Jr.:

The role of common carotid artery velocity patterns in the evaluation of carotid bifurcation disease.

Archives of Surgery 117: $58,1982$.

36. Bresllau, P.J.; Fell, G.; Miller, D.W.; Irey, T.D.; Bailey, W.W.;

Strandness, D.E. Jr.:

Inciclence of carotid arterial disease in patients undergoing coronary bypass surgery.

Stroke 12: 9, 1981.

37. Breslau, P.J.; Knox, R.; Phillips, D.J.; Beach, K.; Chikos, P.M.;

Thiele, B.L.; Strandness, D.E. Jr.:

The accuracy of ultrasonic Duplex scanning as compared with contrast arteriography in extracranial carotid artery disease.

Vascular Diagnosis and Therapy, in press.

38. Brockenbrough, E.C.:

Screening for the prevention of stroke: Use of a Doppler Flowmeter.

Wash./Alaska Regional Medical Programme Brochure, 1970.

39. Bruins Slot, H.:

Doppler studies in the femoro-popliteal pathway.

Thesis, Maastricht, The Netherlands, 1981.

40. Burton, A.L.:

Physiology and Biophysics of the Circulation.

Year Book Medical Publishers Inc. 1972.

41. Chikos, P.M.; Fischer, L; Hirsch, J.H.; Harley, J.D.; Thiele, B.L.;Strandness, D.E. Jr.:

Observer variability in evaluating extracranial carotid artery stenosis.

Stroke, submitted. 
42. Coghlan, B.A.; Taylor, M.G.; King, D.H.:

On-line display of Doppler shift spectra by a new time compression analyzer. In: Cardiovascular Applications of Ultrasound, Ed, : R.S. Reneman, NorthHolland Publ. Co., Amsterdam, 1974.

43. Coghlan B.A.; Taylor, M.G.:

Directional Doppler techniques for detection of blood velocities.

Ultrasound in Med. and Biol. 2: 181, 1976.

44. Cooperberg, P.L.; Robertson, W.D.; Fry, P.; Sweeny, V.:

High resolution real time ultrasound of the carotid bifurcation.

Journal of Clinical Ultrasound 7: 13, 1979.

45. Cossman, D.; Callow, A.D.; Stein, A.; Matsumoto, G.:

Early restenosis after carotid endarterectomy.

Archives of Surgery 113:275, 1978 .

46. Crawford, E.S.; Palamara, A.E.; Kasparian, A.S.:

Carotid and noncoronary operations: Simultaneous, staged and delayed

Surgery 87: 1, 1980.

47. Croft, R.J.; Ellam, L.D.; Harrison, M.J.G.:

Accuracy of carotid angiography in the assessment of atheroma of the internal carotid artery.

Lancet I: $997,1980$.

48. Curry, G.R.; White D.N.:

Color coded ultrasonic differential velocity arterial scanner (Echoflow).

Ultrasound in Med. and Biol. 4: 27, 1978.

49. David, T.E.; Humphries, A.W." Young, T.R.; Bevan, E.G.:

A correlation of neck bruits and arterioscle rotic carotid arteries.

Archives of Surgery 107: 729, 1973.

50. Diaz, F.G.; Patel, S.; Boulos, R; Ausman, J.I.:

Early angiographic changes lollowing carotid endarterectomy.

Stroke 11: 135, 1980 .

51. De Weese, J.A.; May, A.G.; Lipchik, E.O.; Rob, Ch.G.:

Anatomic and hemodynamic correlations in carotid artery stenosis.

Stroke I: $149,1970$.

52. Duncan, G.W.; Gruber, J.O.; Dewey, C.F. Jr.; Meyers, G.S.; Lees, R.S.:

Evaluation of carotid stenosis by phonoangiography.

N. Eng. J. Med. 293: 1124, 1975. 
53. Dyken, M.L.; Klatte, E. "Kolar, O.J.; Spurgeon, C. :

Complete occlusion of common or internal carotid arteries.

Archives of Neurology $30: 343,1974$.

54. Edwards, J.H.; Kricheff, J.J.; Riles, T.; Imparato, A.:

Angiographically undetected ulceration of the carotid bifurcation as cause of embolic stroke.

Radiology 132:369, 1979.

55. Eikelboom, B.C.; Moll, F.L.; Vermeulen, F.E.E.:

Ocular pneumoplethysmography (OPG-Gee).

Proceedings of the symposium on noninwasive diagnostic procedures in extracranial cerebrovascular disease.

Utrecht, The Netherlands, 1980.

56. Eikellooom, B.C.:

Evaluation of carotid artery disease and potential collateral circulation by ocular pneumoplethysmography.

Thesis, Utrecht, The Netherlands, 1981.

57. Eisenberg, R.L.; Nemzek, W.R.; Moore, W.S.; Mani, R.L.:

Relationship of transient ischemic attacks and angiographically demonstrable lesions of the carotid artery.

Stroke 8: $483,1977$.

58. Ennix, C.L.; Lawrie, G.M.; Morris, G.C.; Crawford, E.G.; Howell, J.F.;Reardon, M.J.; Weatherford, S.C.:

Improved results of carotid endarterectomy in patients with symptomatic coronary disease: An analysis of 1546 consecutive carotid operations.

Siroke 10: 122, 1979.

59. Fell, G.; Phillips, D.J.; Chikos, P.M.; Harley, J.O.; Thiele, B.L.;Strandness, D.E. Ir.:

Ultrasonic Duplex Scanning for disease of the carotid artery.

Circulation 64: 1195, 1981.

60. Fell, G.; Breslau, P.J.; Knox, R; Phillips, D.J.; Thiele, B.L.;

Strandness, D.E. Jr.:

The impact of noninwasive testing on the evaluation of patients with asymptomatic carotid bruits.

American Heart Journal $102: 221,1981$.

61. Fields, W.S.; Lemak, N.A.; Frankowksi; Hardy, R.J.:

Controlled trial of aspirin in cerebral ischemia.

Stroke 8: $301,1977$. 
62. Fields, W.S.:

Selection of patients with ischemic cerebrovascular disease for arterial surgery.

World Jourmal of Surgery 3: 147, 1979.

63. Fieschi, C.; Agnoli, A.; Calbo, E.:

Effects of carbon dioxide on cerebral hemodynamics in normal subjects and in cerebrovascular disease studied by carotid injection of radioalbumin.

Circulation Research 13:436, 1968.

64. Fisher, C.M.:

The circle of Willis: Anatomical variations.

Vascular Disease 2:99, 1965

65. Ford, J.; Baker, W.H.; Ehrenhaft, J.L.*

Carotid endarterectomy for nonhemisferic transient ischemic attacks.

Achives of Surgery 110: 1314, 1975 .

66. Forster, F.K.:

The applications and limitations of Doppler spectral broadening measurements for the detection of cardiovascular disorders.

In: Ultrasound in Med., Ed.: D. White, Plenum Press, 1977.

67. French, B.N.; Rewcastle, N.R.:

Recurrent stenosis at site of carotid endarterectomy.

Stroke 8: $597,1977$.

68. Gee, W.; Oller, D.W.; Wylie, E.J.:

Noninvasive diagnosis of carotid occlcusion by ocular pneumoplethysmography. Stroke 7: 18, 1976.

69. Gee, W.; Oller, D.W.; Amundsen, D.G.; Goodreau, J.J.:

The asymptomatic carotid bruit and the ocular pneumoplethysmography. Archives of Surgery 1 12:1381, 1.977 .

70. Gee, $W_{*}$ :

Physiologic principles of ocular pneumoplethysmography.

In: noninvasive diagnostic techniques in vascular disease, Ed.: E. Bernstein, Mosby, St Louis, 1978 .

71. Giddens, D.P.; Mason, R.F.; Cassanca, R.A.:

Measurement of disordered flows distal to subtotal vascular stenosis in the thoracic aortas of dogs.

Circulation Research 39: 112, 1976. 
72. Gosling, R.G.; King, D.H.:

Continuous wave ultrasound as an alternative and complement to $x$ rays in vascular examinations.

In: Cardiovascular Application of Ultrasound, Ed.: R.S. Reneman, NorthHolland, Publ. Co., 1974.

73. Greene, F.M. Jr.; Beach, K.W.; Strandness, D.E. Jr; Fell, G.; Phillips, D.S.: A complete based pattern recognition approach to quantification of carotid arterial disease using pulsed Doppler ultrasound.

Ultrasound Med. and Biol., in press.

74. Hammond, J.H.; Elsinger, R.P.:

Carotid bruits in 1000 normal subjects.

Archives of Internal Medicine 109: 563, 1962.

75. Hass, W.K.; Field, W.S.; North, R.R.; Kricheff, I.I.; Chase, N.E.;Bauer, R.B.: Joint Study of Extracranial Arterial Occlusion. II. Arteriography, Techniques, Sites and Complications.

JAMA 203: 159, 1968.

76. Hartley, C.J.; Strandness, D.E. Jr.:

The effect of atherosclerosis on the transmission of ultrasound. Journal of Surgical Research 9: 575, 1969.

77. Harris, F.J.:

Trigonometric transforms a unique introduction to FFT.

Spectral Dynamics Corp. Tech. pub. DSP-005, 1977.

78. Haynes, C.D.; Dempsey, R.L.:

Carotid endarterectomy. Review of 276 cases in a community hospital.

Annals of Surgery 189: 758, 1979.

79. Hertz, C.H.:

In: Handbook of clinical ultrasound, Pulse-echo techniques.

Ed.: M. de Vlieger, John Wiley and Sons, 1978.

80. Hessel, S.J.; Adams, D.F.; Abrams, H.L.:

Complications of angiography.

Radiology 138: 273, 1981 .

81. Hetzer, N.R.; Loop, F.D.; Taylor, P.C.; Beven, E.G.:

Staged and combined surgical approach to simultaneous and carotid coronary vascular disease.

Surgery: $84,803,1978$. 
82. Heyman, A; Wilkinson, W.E.; Heyden, S.; Helns, M.J.; Bartel, A.G.; Karp, H.P.; Tyroler, H.A.; Curtis, G.H.:

Risk of stroke in asymptomatic persons witl cervival bruits.

N. Eng. J. Med. 302: 838, 1980.

83. Hobson, R.W.; Berry, S.M.; Katocs, A.S. et al.:

Comparison of pulsed Doppler and real-time B-mode echo arteriography for noninvasive imaging of the extracranial carotid arteries.

Surgery $87: 286,1980$.

84. Hoeks, A.P.G.; Reneman, R.S.; Ruissen, C.J.; Smeets, F.A.M.:

Possibilities and limitations of pulsed Doppler systems.

In: Echocardiology. Ed.: C. Lancée. Martinus Nijhoff Publ. "The Hague, 1979.

85. Hoeks, A.P.G.; Reneman, R.S.; Peronneau, P.A.:

A multigated pulsed Doppler system wiih serial data processing.

IEEE Trans. on Sonics and Ultrasonics, 4: 242, 1981.

86. Hoeks, A.P.G.:

On the development of a multigated pulsed Doppler system with serial data-processing.

Thesis, Maastricht, The Netherlands, 1982.

87. Hokanson, D.E.; Mozersky, D.J.; Sumner, D.S.; Strandness, D. E. Jr.:

Ultrasonic arteriography: A new approach to arterial visualization.

Biomed. Engr. 6: 420, 1971.

88. Hokanson, D.E.; Mozersky, D.J.; Sumner, D.S.; McLeod, F.D,;

Strandness, D.E. Jr. :

Ultrasonic arteriography: A noninvasive method for arterial visualization.

Radiology 102: 435, 1972 .

89. Humphries, A.W.; Young, J.R.; Santilli, P.H.; Beven, E.G.; de Wolfe, V.G.:

Unoperated, asymptomatic significant internal carotid artery stenosis: A review of 182 instances.

Surgery $80: 695,1976$.

90. Hutchison, K.J.; Thiele, B.L.; Greene, F.M.; Strandness, D.E. Jr.:

Detection of disturbed flow by computer processing of pulsed Doppler spectra.

In: Theory and Practices of Blood Flow Measurement. Ed.: A. Stewens, in press.

91. Javid, H.; Ostermiller, W.E.; Hengesh, J.W.; Dye, W.S.; Hunter, J.A.;Najafi, H.;

Julian, O.C.:

Natural history of carotid bifurcation atheroma.

Surgery 67: 80, 1970 . 
92. Johnston, K.W.; Maruzzo, B.C.; Cobbold, R.S.C.:

Inaccuracies of a zero crossing detector for recording Doppler signals.

Surgical Forum 28: $201,1977$.

93. Jorgensen, J.E,; Campan, D.N.; Baker, D.W.:

Physical characteristics and mathematical modeling of the pulsed ultrasonic flowmeter.

Med. and Biological Engineering 11: 404, 1973.

94. Kartchner, M.M.; McRae, L.P.; Morrison, F.D.:

Noninvasive detection and evaluation of carotid occlusive disease.

Archives of Surgery 106: $528,1973$.

95. Kartchner, M.M.; McRae, L.P.; Crain, V.; Withaker, B.:

Oculoplethysmography: An adjunct to arteriography in the diagnosis of extracranial carotid occlusive disease.

The American Journal of Surgery 132: 728, 1976.

96. Kartchner, M.M.; McRae, L.P.:

Noninvasive evaluation and management of the "asymptomatic" carotid bruit. Surgery $82: 840,1977$.

97. Keagy, B.A.; Pharr, W.F.; Thomas, D.D.; Bowes, D.E.:

Oculoplethysmography/carotid phono-angiography.

Archives of Surgery 115: 1199, 1980.

98. Keller, H.M.; Meier, W.E.; Anliker, M.; Kumpe, D.A.:

Noninvasive measurement of velocity profiles and bloodflow in the common carotid artery by pulsed Doppler ultrasound.

Stroke $7: 370,1976$.

99. Kety, S.S.; Schmidt, C.F.:

The effects of altered arterial tensions of carbon dioxide and oxygen on cerebral blood llow and cerebral oxygen consumption of normal young men.

J.Clin. Invest. $27: 484,1948$.

100. Kiser, K.M.; Falsetti, H.L.; Yu, K.H.; Resitarits, M.R.; Francis, G.P.;Carroll, R.J.:

Measurement of velocity waveforms in the dog aorta. Journal of fluids engineering: 297, 1976.

101. Kistler, J.P.; Lees, R.S.; Miller, A.; Crowell, R.M.; Roberson, G.: Correlation of spectral phono-angiography and carotid angiography with gross pathology in carotid stenosis.

N. Engl. J. Med. 305: 417, 1981. 
102. Knox, R.; Breslau, P.J.; Chikos, P.M.; Strandness, D.E. Jr.:

The collateral blood supply to the brain.

In preparation.

103. Knox, R.A.; Phillips, D.J.; Breslau, P.J.; Lawrence, R.; Primozich, J; Strandness, D.E. Jr.:

Empirical findings relating sample volume size to diagnostic accuracy in pulsed Doppler cerebrovascular studies.

Journal of Clinical Ultrasound, in press.

104. Knox, R.; Breslau, P.J.; Strandness, D.E. Jr.:

Quantitative carotid phono-angiography.

Stroke 12: 798, 1981 .

105. Knox, R.; Breslau, P.J.; Strandness, D.E. Jr.:

A simple parameter for accurate detection of severe carotid disease.

British Journal of Surgery, in press.

106. Kremen, J.E.; Gee, W.; Kaupp, H.A.; McDonald, K.M.:

Restenosis or occlusion after carotid endarterectomy.

Archives of Surgery 114: 608, 1979.

107. Lees, R.C.; Dewey, C.F.*

Phono-angiography: A new noninvasive diagnostic method for studying arterial disease.

Proct. Matl. Acad. Sci. USA 67: 935, 1970.

108. Levy, R.J.:

Stroke decline: Implications and Prospects.

N. Eng. J. Med. 300: 490, 1979.

109. Lewis, R.R.; Beasley, M.G.; Hyams, D.E.; Gosling, R.G.:

Imaging the carotid bifurcation using continuous-wave Doppler-shift ultrasound and spectral analysis.

Stroke 9: 465, 1978.

110. Light, L.H.:

A recording spectrograph for analysis Doppler blood velocity signals in real-time. Journal of Physiology 207: 42, 1971.

111. Ling, S.C.; Atabek, H.B.; Fry, D.L.; Patel, D.J.; Janicki, J.S.:

Application of heat-film velocity and shear probes to hemodynamic studies.

Circulation Research 23: 789, 1968.

112. Ling, S.C.; Letzing, W.G.; Patel, D.J.:

Non-linear analysis of aortic flow in living dogs.

Circulation Research 33: 198, 1973. 


\section{Lunt, M.J.:}

Accuracy and limitations of the ultrasonic Doppler blood velocimeter and zero crossing detector.

Ultrasound in Med. and Biol. 2: 1, 1975.

114. Lye, Ch.R.; Sumner, D.S.; Strandness, D.E. Jr.:

The accuracy of the supraorbital Doppler examination in the diagnosis of hemodynamically significant carotid occlusive disease.

Surgery 79: 42, 1976.

\subsection{Lyons, C..:}

Progress report of the Joint Study of Extracranial Arterial Occlusion.

In: Cerebral Vascular Diseases. Ed.: R. Siekert. Grune and Stratton, 1965.

116. Malone, J.M.; Bean, B.; Laguna, J.; Hamilton, R.; Labadie, E.; Moore, W.S.:

Diagnosis of carotid artery stenosis.

Annals of Surgery 191: 347, 1980.

117. May, A.G.; Van den Berg, L.; De Weese, J.A.; Rob, C.G. :

Critical arterial stenosis.

Surgery $54: 250,1963$.

118. McDonald, D.A.:

Blood flow in arteries.

Camelot Press Litd, 1974.

119. McNamara J.C.; Heyman A.; Silver, D.; Mandel, M.E.:

The value of carotid endarterectomy in treating transient cerebral ischemia of the posterior circulation.

Neurology 27: $682,1977$.

120. Miyazaki, M.; Kato, K.:

Measurement of cerebral blood flow by ultrasonic Doppler technique.

Japanese Circulation Journal 29: 375, 1965.

121. Miyaraki, M.:

Studies on cerebral circulation by ultrasonic Doppler technique with special reference to clinical application of the technique.

Progress in Brain Research 35: 1, 1972.

122. Mol, J.M.F.A.:

Doppler haematotachographic investigation in cerebral circulation disturbances.

Thesis, Utrecht, The Netherlands, 1973.

123. Moniz, E.:

Hémiplegies pour trombose de la carotide interne.

La Presse Medicale 52: 977. 1937. 
124. Mozersky, D.J.; Hokanson, D.E.; Baker, D.W.; Sumner, D.S.;

Strandness, D.E. Jr.:

Ultrasonic arteriography.

Archives of Surgery 103: 663, 1971.

125. Mozersky, D.J.; Hokanson, D.E.; Sumner, D.S.; Strandness, D.E. Jr.: Ultrasonic visualization of the arterial lumen.

Surgery $72: 253,1972$.

126. Mungas, J.E.; Baker, W.H.:

Amaurosis fugax.

Stroke 8: 232, 1977.

127. Nippa, J.H.; Hokanson, D.E.; Lee, D.R.; Sumner, D.S.;

Strandness, D.E. Jr.:

Phase rotation for separating forward and reverse blood velocity signals.

IEEE Trans. on Sonics and Ultrasonics 5: 340, 1975.

128. Novack, P.; Shenkin, H.A.; Bortin, L.; Goluboff, B.; Soffe, A.M.:

The effects of carbon dioxide inhalation upon the cercbral blood flow and cerebral oxygen consumption in vascular disease.

J. Clin. Invest. 32: 696, 1953.

129. O'Donell, T.F.; Parker, S.A.; Callow, A.D.; Kelly, J.J.; McBride, K.J.; Korwin, S.:

The relative value of carotid noninvasive testing as determined by receiver operator characteristic.

Surgery $87: 9,1979$.

130. Olinger, C.P.:

Ultrasonic carotid echoarteriography.

Am. J. Roentgen. Rad. Ther. Med. 106: 282, 1969.

131. Patterson, J.L.; Heyman, A.; Battey, H.L.; Ferguson, R.W.:

Threshold response of the cerebral vessels of man to increase in blood carbon dioxide.

J. Clin. Invest. 34: 1857, 1955.

132. Phillips, D.J.; Blackshear, W.M. Jr.; Baker, D.W.; Strandness, D.E. Jr.: Ultrasound Duplex scanning in peripheral vascular disease.

Radiol. Nuc. Med.: 6, 1978.

133. Phillips, D.J.; Powers, J.E.; Eyer, M.K.; Blackshear, W.M. Jr.

Bodily, K.C.; Strandness, D.E. Jr.; Baker, D.W.:

Detection of peripheral vascular disease using the Duplex scanner III.

Ultrasound Med. and Biol. 6: 205, 1980. 
134. Planiol, T; Pourcelot, L; Pottier, J.M.; Degiovanni, E.:

Etude de la circulation carotidienne par le method ultrasonic et la thermographie. Rev. Neurol. 126: 127, 1972.

135. Planiol, T.H.; Pourcelot, L.:

Diagnostic des tromboses stenoses carotidiennes: Par le effect Doppler.

Second World Congress on Ultrasonics in Medicine, Rotterdam, 1973.

136. Powers, J.IE.:

An ultrasonic annular array based on quadrature sampling.

Thesis University of Washington, U.S.A. 1980.

137. Reid, J.M.:

Principles of Doppler ultrasound.

In: Handbook of Clinicall Ultrasound. Ed.: M. De Vlieger, John Wiley and Sons, 1978.

138. Reid, J.M.; Spencer, M.P.:

Ultrasonic Doppler technique for imaging blood vessels.

Science 176: 1235, 1972.

139. Reneman, R.S.; Spencer, M.P.:

Local Doppler audio spectra in normal and stenosed carotid arteries in man.

Ultrasound Med. and Biol. 5: 1, 1979.

140. Rennie, L.; Ejrup, B.; McDowell, F.:

Arterial bruits in cerebrovascular disease.

Neurology 14: 751, 1964.

141. Reul, G..J.; Morris, G.C.; Howell, J.F.; Crawford, E.S.; Stelter, W.J.:

Current concepts in coronary artery surgery.

Annals of Thorac. Surgery 14:243, 1972.

142. Reynolds, 0. :

On experimental investigation of the circumstances which determine whether the motion of water shall be direct or sinuous, and of the law of resistance in parallel channels.

Phil. Trans. Roy. Soc. London 174: 935, 1883

143. Riles, T.S.; Lieberman, A.; Kopelman, J.; Imparato, A.M.:

Symptoms, stenosis and bruit.

Archives of Surgery 116:213, 1981.

144. Risberg, T.; Smith, P.:

Prediction of hemispheric blood flow from carotid velocity measurements: A study with the Doppler and 133xe inhalation techniques.

Stroke 11:399, 1980. 
145. Rittgers, S.E.; Putney, W.W.; Barnes, R.W.:

Real-time spectrum analysis and display of directional Doppler ultrasound blood velocity signals.

IEEE Trans. on Biomedical Engineering 12: 723, 1980.

146. Rittenhouse, E.A.; Strandness, D.E. Jr.:

Oscillary flow patterns in patients with aortic valve disease.

The American Journal of Cardiology 28: $568,1971$.

147. Rittenhouse, E.A.; Maixner, W.; Burr, J.W.; Barnes, R.W.:

Directional arterial flow velocity: A sensitive index of changes in peripheral vascular resistance.

Surgery $79: 350,1976$.

148. Rutherford, R.B.; Hiatt, W.R.; Kreutzer, E.W.:

The use of velocity wave form analysis in the diagnosis of carotid artery occlusive disease.

Surgery 82: 695, 1977 .

149. Satomura, S.:

Study of flow patterns in peripheral arteries by ultrasonics.

J. Acoust. Soc. Jap. 15: 151, 1959.

150. Schutz, H,; Fleming, J.F. R.; Awerbuck, B.:

Arteriographic assessment of carotid endarterectomy.

Annals of Surgery 171:509, 1970.

151. Seldinger, S.I.:

Catheter replacement of the needle in percutaneous arteriography.

Acta Radiologica 39: $368,1953$.

152. Sokoloff, H.:

The effects of carbon dioxide on the cerebral circulation.

Anesthesiology 21:664, 1960 .

153. Spencer, M.P.; Reid, J.M.; Davis, D.L.; Paulson, P.S.:

Cervicat carotid imaging with a continuous wave Doppler flowmeter.

Stroke 5: 145, 1974.

154. Staiger, J.; Adler, C.P.; Dieckman, H.; Barmeyer, J.:

Postmortem angiographic and pathologicanatomic findings in coronary heart disease.

Cardiovasc. Intervent. Radiol. 3: 139, 1980.

155. Stoney, R.J.; String, S.T.:

Recurrent carotid stenosis.

Surgery 80: 705, 1976. 
156. Strandness, D.E. Jr.; Kennedy, J.W.; Judge, T.P.; McCleod F.D.:

Transcutaneous directional flow detection: A preliminary report.

American Heart Joumal 78: 65, 1969.

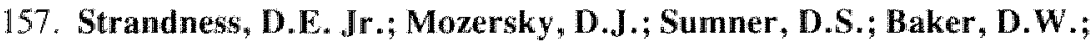
Hokanson, D.E.:

Noninvasive arteriography: A new approach for arterial visualization.

The American Surgeon 38: 494, 1972.

158. Strandness, D. E. Jr.:

Evaluation of the patient for vascular surgery.

Surgical Clinics of North America 54: 13, 1974.

159. Strandness, D.E. Jr.; Sumner, D.S.:

Hemodynamics for surgeons.

Grune and Stratton, New York, 1975.

160. Strandness, D.E. Jr.:

The use and abuse of vascular laboratory.

Surgical Clinics of North America 59: 707, 1979.

161. Strandness, D.E. Jr.; Fell G.:

Doppler Duplex Scanning proceedings of the symposium on noninvasive

diagnostic procedures in extracranial cerebrovascular disease.

Utrecht, The Netherlands, 1980.

162. Strother, C.M.; Sackett, J.F., Crummy, A.B.;

Turnipsed, W.M. et al.:

Clinical applications of computerized fluoroscopy.

Radiology 136:781. 1980.

163. Sumner, D.S.; Russell, J.B.; Ramsey, D.E. ; et al.:

Noninvasive diagnosis of extracranial carotid arterial disease.

Archives ofl Surgery 1 14: 1.222, 1979.

164. Swanson, P.D.; Calanchini, P.R.; Dyken, M.L.; Gotshall, R.D.;

Haever, A.F.; Poskanzer, D.C.:

A cooperative study of hospital frequency and character of transient ischemic atlacks.

JAMA 237: 2202, 1977 .

165. Thiele, B.L.; Hutchinson, K.J.; Forster, F. K.; Strandness, D.E. Jr.:

Pulsed Doppler velocity wave form patterns produced by smooth stenosis in the dog thoracic aorta.

In: Theory and practices of blood flow measurements. Ed.:E. Stevens, in press. 
166. Thiele, B.L.; Young, I.V.; Chikos, P.M.; Hirsch, J.H.;

Strandness, D.E. Jr.:

Correlation of arteriographic findings with symptoms in patients with

Cerebrovascular disease.

Neurology $30: 1041,1980$.

167. Thompson, J.E.; Patman, R.D.; Persson, A.V.:

Management of asymptomatic carotid bruits.

The American Surgeon 42: 77, 1976.

168. Thompson, J.E.; Kartchner, M.M.; Austin, D.J.; Wheeler, C.G.;

Patman, R.D.:

Carotid endarterectomy for cerebrovascular insufficiency (stroke): Follow-up of 359 cases.

Antuals of Surgery 163:751, 1966.

169. Thompson, J.E.; Austin, D.J.; Patman, R.D.:

Carotid endarterectomy for cerebrovascular insufficiency: longterm results in 592 patients followed up to thirteen years.

Annals of Surgery 172:663, 1970.

170. Thompson, J.E.; Patman, R.D.; Talkington, C.M.:

Asymptomatic carotid bruit: Long-term outcome of patients having endarterectomy compared with unoperated controls.

Annals of Surgery 188: $308,1978$.

171. Thompson, J.E.:

Complications of carotid endarterectomy and their prevention.

World Journall of Surgery 3: 155, 1979.

172. Toole, J.F.:

Management of transient ischemic attacks.

In: Cerebrovascular Disease, P. Scheinberg Raven Press, New York, 1976.

173. Turnipseed, W.D.; Berkhoff, H.A.; Crummy, A.:

Postoperative occlusion after carotid endarterectomy.

Archives of Surgery 115: 573, 1980.

174. Turnipseed, W.D.; Berkoff, H.A.; Belzer, F.O.:

Postoperative stroke in cardiac and peripheral vascular disease.

Annals of Surgery $192: 365,1980$.

175. Turnipseed, W.D.; Crummy, A.B.; Strother, C.; et al.:

Computerized intravenous arteriography: A technique for visualizing the peripheral vascular system.

Surgery 89: 118, 1981 . 
176. Turnipseed, W.D.; Sackett, J.F.; Strother, C.M.; Crummy, A.B.;

Mistretta, C.A., Kruger, R.A.:

Computerized arteriography of the cerebrovascular system.

Archives of Surgery 1 16: 470,1981.

177. Weaver, R.G.; Howard, G.; McKinney, W.M.; Ball, M.R.; Jones, A.M.

Toole, il. F.:

Comparison of Doppler ultrasonography with arteriography of the carotid artery bifurcation.

Stroke 11: 402, 1980 .

178. White, D.M.; Curry, G.R.:

A comparison of 424 carotid bifurcations examined by angiography and the Doppler Echoflow.

Ultrasound in Medicine 4: 363, 1978.

179. White, J.S.; Sirinek, K.R.; Root, H.D.; Rogers, W.:

Morbidity and mortality of carotid endarterectomy.

Archives of Surgery 116: 409,1981 .

180. Whitemore, R.L.:

Rheology of the Circulation, Pergamon Press, New York, 1968.

181. Wolf, P.A., Kannel, W.B.; McNamara P.M.; Dawber, T.R.:

Asymptomatic carotid bruits and risk for stroke.

Stroke 10: $96,1979$.

182. Wolf, P.A.; Kannel, W.B.; Sorlie, P; McNamara, P.:

Asymptomatic carotid bruit and risk of stroke.

JAMA 215:1442, 1981 .

183. Wylie, E.J.; Ehrenfeld, W.K.:

Extracranial occlusive cerebrovascular disease. Saunders, New York, 1970.

184. Yao, S. T.; Needham, T.N.:

Frequency malysis of Doppler shift blood flow signals by a bandpass filter:

Preliminary report.

Biomed. Eng. 5: 438,1970 .

185. Ziegller, D.K.; Zileli, T.; Dick, A.; Sebaugh, J.L.:

Correlation of bruits over the carotid artery with angiographically demonstrated lesions.

Neurology 21: 860, 1971 


\section{Acknowledgements}

Many people in Seattle and Maastricht have been involved in realizing this thesis, I am grateful for all their support.

I would like to express my gratitude especially to Professor J. M Greep, who made it possible for me to spend a year in Seattle to work on the subject of my choice under the most desirable conditions.

I would also like to express my gratitude towards Professor D.E. Strandness, who provided me with every support that I needed to complete the work compiled in this thesis. You and your family made the year Liesbeth and I spent in Washington one of the most productive and happiest in our lives. We greatly appreciate your warm friendship.

I feel grateful to Professor J.M. Greep. Professor R.S. Reneman and Professor H. A.J. Lemmens for their critical contributions to this work.

I would like to express my deep appreciation towards David Phillips and Kirk Beach. They introduced me into the wonderful world of ultrasound, their friendly personalities and technical skills have been of great support to me.

I thank my colleagues in the vascular laboratory of the Department of Surgery:

Gary Fell, Robin Knox and Yves Langloise for their pleasant collaboration and friendship.

Special thanks must be reserved for Jeanny Primozich and Ramona Lawrence, their excellent work in the carotid laboratory has been the basis for the results described in this thesis.

I like to thank Cees van der Linden and Paul Jörning for their corrections, and Chris Voskamp for his high quality illustrations and preparation of the lay-out. I feel much obliged to Maartje Duyzings for her courage, patience and intelligence in typing the manuscript.

Finally, I like to thank my parents for the confidence they always had in their son, and Liesbeth who helped me much more than she realized. 


\section{Curriculum Vitae}

Paul Breslau was born on the 13th of September 1946 in The Hague, The Netherlands. After graduating from high school in The Hague, he served the Royal Dutch Army. In 1969 he went to Medical School at the University of Leyden and graduated in 1975. In 1976 he started his surgical residency training at the Department of Surgery of the St. Annadal Hospital, University of Limburg (Head: Prof. Dr. J.M. Greep).

From April 1980 till May 1981 his surgical residency was interrupted by a research fellowship at the Department of Surgery of the University of the Washington Hospital, Seattle, U.S.A. (Head: Prof. D.E. Strandness, Jr., MD).

From May 1981 on, his surgical residency program has been resumed at the Department of Surgery of the St. Annadal Hospital, University of Limburg, The Netherlands. 